\title{
A paired neural network model for tourist arrival forecasting
}

\begin{abstract}
Tourist arrival and tourist demand forecasting are a crucial issue in tourism economy and the community economic development as well. Tourist demand forecasting has attracted much attention from tourism academics as well as industries. In recent year, it attracts increasing attention in the computational literature as advances in machine learning method allow us to construct models that significantly improve the precision of tourism prediction. In this paper, we draw upon both strands of the literature and propose a novel paired neural network model. The tourist arrival data is decomposed by two low-pass filters into long-term trend and short-term seasonal components, which are then modelled by a pair of autoregressive neural network models as a parallel structure. The proposed model is evaluated by the tourist arrival data to United States from twelve source markets. The empirical studies show that our proposed paired neural network model outperforming the selected benchmark model across all error measures and over different horizons.
\end{abstract}

\section{Introduction}

Tourist flows, regardless the sources and destinations, are all observed seasonally variant. The seasonality is one of the most critical features of the tourism industry and have an important impact on the local tourism economy, which is strongly correlated with the macroeconomic planning, operation and resource allocations in local community. The advantages and disadvantages of the seasonal patterns on local economic development, domestic business, and the macroeconomics have been thoroughly studied in (Butler, 2001) (Lim \& McAleer, 2001) and (Chen, Li, Wu, \& Shen, 2017). The advantages include the "normal" life style of the local people in low season and the maintenance and recovery of the natural and municipal resources out of the peak season. Nevertheless, the disadvantages include the over-utilization of the resources and infrastructures during peak seasons due to the overwhelming tourist demand and consumption, which may challenge the management of the investment and labour employment for the local government (Butler, 2001) (Chen, Li, Wu, \& Shen, 2017). Consequently, tourist arrivals from different source markets, and the arrival forecasting, plays a crucial role in tourism industry, and in the local economy as well. Accurately forecasting tourist arrivals (demands) from every single source is of huge important to the researchers, industry practitioners, and decision-makers. 
In the past decades, a big number of forecasting models have been proposed, applied and tested in the tourism forecasting area. (Chen, $\mathrm{Li}, \mathrm{Wu}, \&$ Shen, 2017) thoroughly reviewed and summarized the models proposed in the last two decades in three primary categories: deterministic seasonality, stochastic seasonality and multivariate time series model. The deterministic seasonality model assumes a seasonal unconditional mean that varies with the seasons of the year. Extensive studies investigate the method that extracts the seasonal component from the demand series to generate a seasonally adjusted series, i.e., moving average has been proposed to separate the seasonal component by (Lim \& McAleer, 2001) but it is not suitable for forecasting. Stochastic seasonality models, which, on the other hand, dominate the area of tourist demand forecasting in recent literatures, include two types: stochastic stationary and non-stationary seasonality, which assume an invariant (Osborn, Harevi, \& Birchenhall, 1999) (Kim, 1999) (Ghysels \& Osborn, 2001) and variant seasonal pattern (Lim \& McAleer, 2001) (Song, Li, Witt, \& Athanasopoulos, 2011) respectively. The latter type, stochastic non-stationary model, has been widely used in recent years and includes two major models: the seasonal autoregressive integrated moving average (SARIMA) family of seasonal unit root models (Alleyne, 2006) (Kulendran \& Wong, 2005) (Oh \& Morzuch, 2005) and the paired time series model (STSM) (Harvey \& Todd, 1983). Both models assume that the tourist arrival data is composed of trend and seasonal components as well as irregular terms. The SARIMA model stabilizes the time series by seasonal and non-seasonal differencing, which is widely applied in financial area for reducing the non-stationarity, while the STSM model implicitly decomposes the time series into stochastic trend, seasonal and irregular terms. Though the SARIMA model is almost the most widely applied model in tourism demand forecasting, the STSM model has been reported to outperform most competing models consistently, including naïve Bayesian, neural network and exponential smoothing models (Kon \& Turner, 2005). In addition, STSM model has been proved to outperform the SARIMA model in (Kulendran \& Witt, 2003) (Song, Li, Witt, \& Athanasopoulos, 2011).

Computational techniques such as machine learning methods, although not studied thoroughly, have also been used in tourism forecasting in a few literatures, among which a few studies confirmed the superior performance of ANN. (Muysal \& Roubi, 1999) initially investigated the possibility of applying artificial neural network (ANN) on forecasting the quarterly Canadian tourism expenditures in United States. By selecting the variables that are relevant to the expenditures as well as the lagged expenditure values as the input of the ANN, this study demonstrated the usefulness of a standard structure ANN in tourism demand forecasting. (Law \& Au, 1999) applied neural network with six 
input neurons with six input variables that are relevant to the service, hotel, foreign exchange rate, and market expenses to forecast the annual Japanese tourist arrivals in Hong Kong. It further confirmed the superior performance to regression, naïve, moving average and exponential smoothing. (Law R. , 2000) further applied and highlighted the importance of incorporating the back-propagation method to train the ANN model in forecasting tourist arrivals. (Kon \& Turner, 2005) further compared the forecasting performance of ANN with basic paired method (BSM), naïve, and Holt-Winter methods by using the Singapore quarterly tourist arrivals from five source markets. They showed that ANN can achieve the best performance by the standard structure. (Burger, Dohnal, Kathrada, \& Law, 2001) applied ANN on monthly tourist arrivals from USA to Durban, South Africa with comparison to widely used competing models and demonstrated that ANN is better to handle non-linear time series than other models.

On the other hand, some other studies showed the traditional models outperforming the ANN. (Claveria \& Torra, 2014) further analysed the performance of ANN in forecasting tourism demand of Catalonia from more than ten source markets. They found that the ARIMA model performed the best in most cases especially in short horizon forecasting while the ANN was the most underperformed model. It is noted that this study used a structure of ANN with one lag input and three hidden neurons, which is usually considered as an over-simple structure and often results in an underfitting problem. This paper also claimed that the structure of ANN might be further improved for a better performance. (Hassani, Silva, Antonakakis, Filis, \& Gupta, 2017) thoroughly evaluated the forecasting performance across seven popular models by the monthly international tourist arrival data in nine European countries across Jan 2000 to Dec 2013. The Singular spectrum analysis (SSA), ARIMA and Trigonometric box-cox ARMA trend seasonal model (TBATS) have been evaluated as the best-performed models while other models including neural network provided the least accurate results. It is noted that the ANN model used in this study contained 2-lagged input and 1 hidden neuron, which is, again, an over-simple structure and is usually underfitting the data. (Pai, Hung, \& Lin, 2014) proposed a new logarithm least-squares support vector regression (LLS-SVR) model and applied in forecasting the tourist arrivals to Taiwan and Hong Kong. LLS-SVR is a revised model based on the well-known computational method: support vector machine (SVM) and it achieved superior performance to those of the traditional methods. It also claimed that ARIMA model is slightly underperformed in capturing the trend of the data and is therefore not suggested for forecasting the tourist arrival data (Pai, Hung, \& Lin, 2014). 
As the studies in (Claveria \& Torra, 2014) and (Hassani, Silva, Antonakakis, Filis, \& Gupta, 2017), the ANN has been applied in tourist arrival data forecasting for the last two decades, it, however, has been treated merely as a black-box with the simplest structure with 1 to 2 lags input and 1 hidden neuron. It is noted that an ANN with a single hidden neuron equipped with sigmoid activation function is functionally equivalent to a logistic regression with bias term, which apparently may not be able to capture the strong non-linearity and seasonality in the tourism data. In addition, previous studies usually directly fed the original tourism data into the ANN model and expected a "magic" forecasted output. Almost no study has thoroughly revised and tailor-made the ANN for a specific application in tourism area with respect to the significantly different patterns in this area.

In this paper, we address this problem by proposing a novel method that makes use of the capability of the computational methods. ANN is strong in capturing the non-linearity of the time series especially the detrend series (Zhang \& Qi, 2005), while autoregressive neural network, may not be strong in modelling the strong seasonal patterns (Zhang \& Qi, 2005) (Patil, Tantau, \& Salokhe, 2008).

Inspired by the work in (Yao, et al., 2017), we propose a methodology specifically for modelling tourism data. We assume the existence of an increasing trend plus a repeated annual seasonality with slightly different amplitudes in the tourism data following the work in (Chen, $\mathrm{Li}, \mathrm{Wu}, \&$ Shen, 2017). We firstly extract the trend and seasonal components explicitly by the mathematical tools in (Harris, Stoja, \& Yilmaz, 2011) (Yao, et al., 2017) subject to certain constraints of the seasonal components. We use the autoregressive neural network (ARNN) to model the extracted trend and seasonal components separately following (Yao, et al., 2017). The contribution of this paper is in two strands. The first is to answer a question: can ANN-based model achieve better performance than traditional models in forecasting tourists. Different from the work in (Claveria \& Torra, 2014) and (Hassani, Silva, Antonakakis, Filis, \& Gupta, 2017), this study proposes an application-specific approach of a pair of autoregressive neural network (ARNN) to model and forecast the tourists. The decomposition tools, stationarity test and two separated ARNNs in the approach provide a statistical-principled manner instead of the heuristics to decompose and model the tourism data. On the other hand, the approach satisfies the assumption of stationary seasonality in tourism studies. This approach, as far as we aware, is the first tailor-made $\mathrm{NN}$ based model according to the intrinsic feature of the tourism data. The evaluations in Section 3 show a consistently better forecasting performance of the proposed approach. The second is a thorough performance study of the proposed approach with a few decomposition methods under heuristic parameter selection, although our approach determines the parameters based on a statistical principle. Explicit decomposed components rely on certain 
parameters of different tools. Selecting appropriate parameter determines the forecasting performance of the whole model. This is as far as we aware, the first comparison study of the wellknown decomposition methods and the corresponding forecasting performances.

The rest of this paper is structured as follows. In Section 2, our proposed model is discussed in detail. In Section 3, the data and the empirical studies are also introduction as well as the analysis of the performance of the out-of-sample forecasting results. The Section 4 concludes.

\section{Model and Research Method}

We propose a simple and effective method to construct a pair of Neural Network ( $p \mathrm{NN})$ for the tourist arrival forecasting. We represent the tourist arrival data as the variable $y_{t}$, and construct $p \mathrm{NN}$ following the form of the basic paired model defined in (Harvey \& Todd, 1983) as

$$
y_{t}=\mu_{t}+\gamma_{t}
$$

where $t=1, \ldots, T, \mu_{t}$ and $\gamma_{t}$ are trend and seasonal components respectively. We assume the seasonal component $\gamma_{t}$ following a stationary autoregressive process of order $s: A R(s)$, where $s$ is the number of "seasons" in a year (Harvey \& Todd, 1983). To maintain such assumption, we do not follow the originally defined form of trend in (Harvey \& Todd, 1983); instead, we assume that $\mu_{t}$ follows a smooth and non-stationary process but leave its precise dynamics unspecified.

The $p \mathrm{NN}$ is a methodological framework containing three steps:

- Step 1: extracting the trend component $\mu_{t}$ from $y_{t}$ explicitly subject to the stationarity of the seasonal component $\gamma_{t}$;

- Step 2: modelling the trend and seasonal component, $\mu_{t}$ and $\gamma_{t}$, by two autoregressive neural networks separately;

- Step 3: generating the $n$-step ahead forecasting results, $\hat{\mu}_{t+n}$ and $\hat{\gamma}_{t+n}$, separately and aggregating them as the final forecasting results as $\hat{y}_{t+n}=\hat{\mu}_{t+n}+\hat{\gamma}_{t+n}$.

We introduce the implementation of $p \mathrm{NN}$ with different techniques and compare their performances. 


\subsection{Step 1: trend and seasonality decomposition}

We implement the explicit extraction of the trend component $\mu_{t}$ from $y_{t}$ by three well-known methods: 1) the low-pass Hodrick-Prescott (HP) filter (Hodrick \& Prescott, 1997); 2) the wavelet transformation (WT) method (Daubechies, 1992); and 3) a simple moving average (MA) method (Lim \& McAleer, 2001). We tune the parameters of those methods to obtain the $\mu_{t}$ subject to the stationarity of the seasonal component $\gamma_{t}$, obtained by $\gamma_{t}=y_{t}-\mu_{t}$. The stationarity of $\gamma_{t}$ is tested and determined through a statistically principled manner: the Augmented Dickey-Fuller (ADF) test (Fuller, 1976), which has the null hypothesis that a unit root is present in the seasonal component. Thus, the extraction of the trend $\mu_{t}$ is a process that optimizes the appropriate parameters of the decomposition methods (i.e., HP, WT, and MA) to satisfy the stationarity constraint of the seasonality $\gamma_{t}$.

The explicit extraction of the trend component is due to three reasons. The first is to satisfy the assumption of a stationary and autoregressive seasonal component, which otherwise was not guaranteed but merely assumed in traditional paired model (Harvey \& Todd, 1983) and seasonal ARIMA model (Kulendran \& Wong, 2005). The second is to statistically and deterministically generate the trend and the seasonal components, which were otherwise not explicitly defined and uneasily to observe (Harvey \& Todd, 1983) (Kulendran \& Wong, 2005). Therefore, the explicit extraction provides an interpretable method that generate the economic meaningful and easily illustrated forecasting results. The third is that we hypothesize that separately modelling two components may achieve better forecasting performance than the traditional models.

\section{Low-pass Hodrick-Prescott (HP) filter}

The HP filter was proposed in (Hodrick \& Prescott, 1997) and is widely applied in macroeconomics for removing the short-run cyclical component and revealing the low-frequency non-linear trend of a time series (Stock \& Watson, 1999) (McElroy, 2008) (Stock \& Watson, 2016). For a time series data $y_{t}$, given the value of the smoothing parameter $\lambda$, the trend component $\mu_{t}$ can be obtained by solving the following:

$$
\min _{\mu_{t}}\left(\sum_{t=1}^{T}\left(y_{t}-\mu_{t}\right)^{2}+\lambda \sum_{t=2}^{T-1}\left[\left(\mu_{t+1}-\mu_{t}\right)-\left(\mu_{t}-\mu_{t-1}\right)\right]^{2}\right)
$$

where the smoothing parameter $\lambda$ penalizes the variation in the growth rate of the trend component $\mu_{t}$. The larger the $\lambda$, the heavier the penalty for variation, and the smoother the $\mu_{t}$. Therefore, the implementation of Step 1 by HP filter is to select an appropriate value of $\lambda$ to maintain the stationarity 
of $\gamma_{t}$. The $\lambda$ value selection is usually started from $\lambda=129600$, the empirical value recommended in (Baxter \& King, 1999) and (Ravn \& Uhlig, 2002), down to $\lambda=0$. For each value of $\lambda$, we apply ADF testing on the $\gamma_{t}$, once the null is rejected, which suggests the seasonal component is stationary, the stationarity condition is then satisfied. We show in Figure 1 an example of selecting the value of $\lambda$ by the HP filter. The data is monthly tourist arrivals of US from UK in the period Jan 1996 to Sep 2017. We start with $\lambda=129600$ in Figure 1(a) and apply ADF test at each value of $\lambda$ until the case in Figure 1(d), where the selection procedure yields a $\lambda=100$ with a highly significant $p$-value at 0.001 for ADF test. Figure 1(b) and (c) are two examples of the intermediate $\lambda$ values 3600 and 1600 of the selection process. We observe a pattern that as the smoothing parameter decreases, the trend component becomes less smooth and the resulting seasonality component slowly turns more into a stationary seasonal process in Figure 1(d), which fulfils the assumption of basic paired time series model proposed in (Harvey \& Todd, 1983).

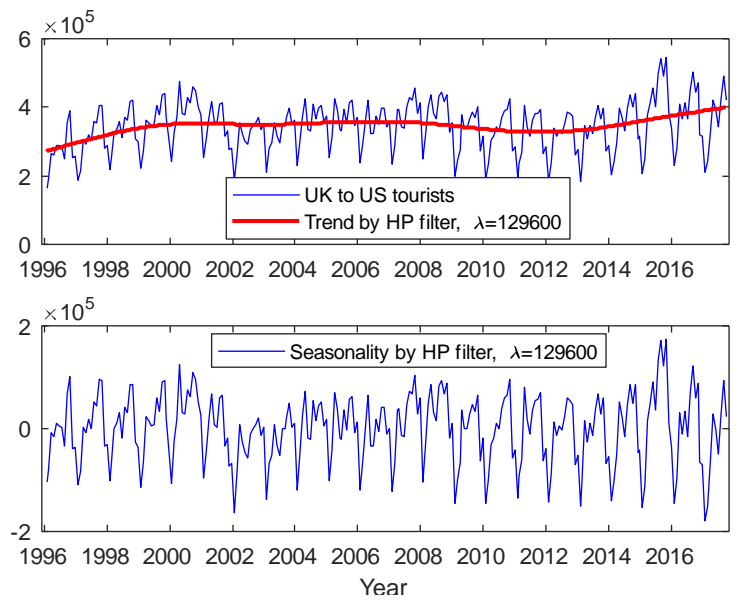

(a)
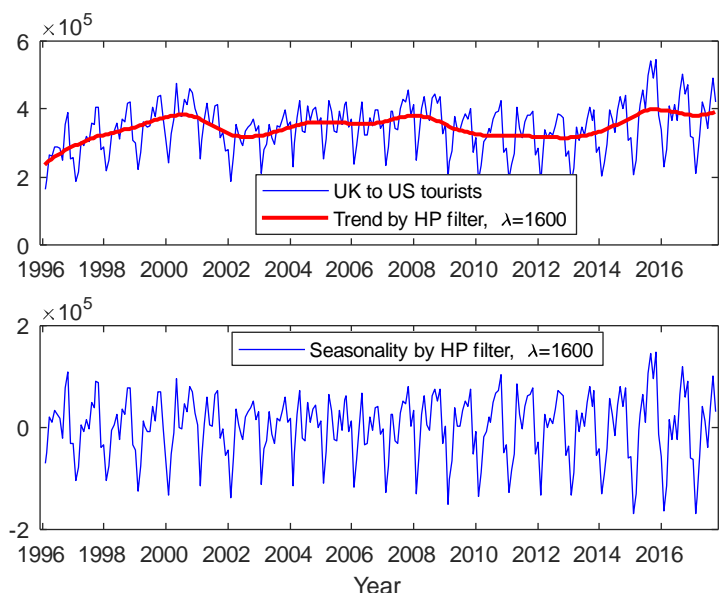

(c)
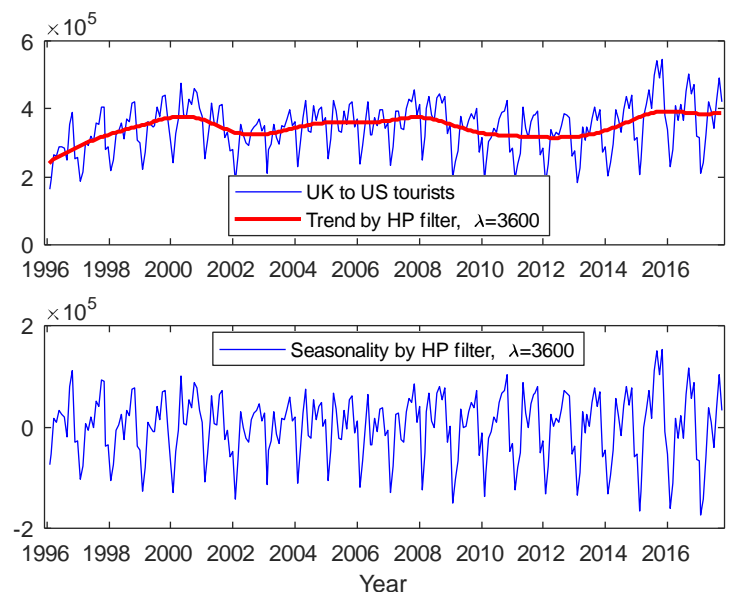

(b)
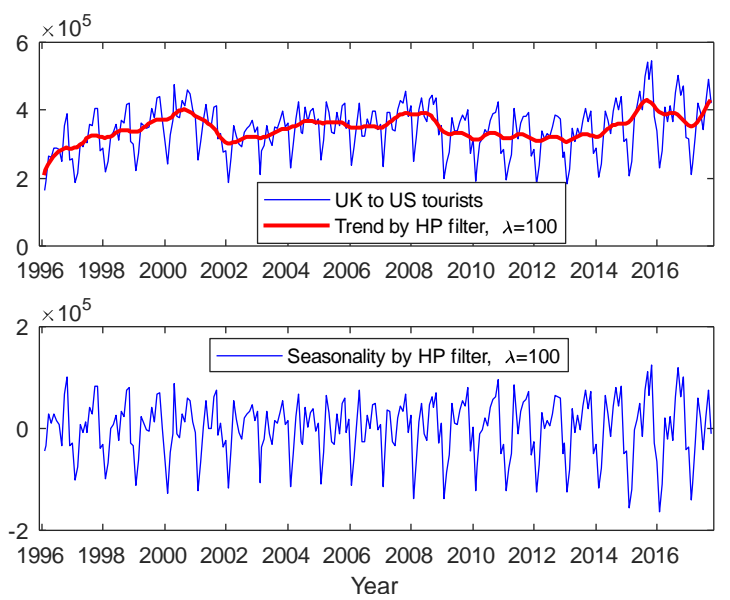

(d)

Figure 1 This figure shows the trend and seasonality decomposition by HP filter with different values of parameter $\lambda$. In upper parts of (a)-(d), blue curves represent the monthly tourist arrival from UK to US from Jan 1996 to Sep 2017 and the red curves are the trend component by HP filter with $\lambda$ value of 129600, 3600, 1600 and 100 respectively. In the lower 
parts of (a)-(d), blue curves are the corresponding seasonal component obtain as the difference between the arrival data and the trend component respectively.

\section{Wavelet transformation (WT) method}

Wavelet analysis has been successfully applied to de-noise the option prices to obtain accurate option-implied risk neutral density (Haven, Liu, \& Shen, 2012) and was also successfully used in volatility forecast (Barunik, Krehlik, \& Vacha, 2016) and high frequency financial data mining (Sun $\&$ Meinl, 2012). A key feature of the wavelet transform is that it can decompose any square integrable function into a combination of some scaling function and wavelet functions, each factored by their corresponding approximation coefficients and detail coefficients. Once the original function has been decomposed at certain level, its detail coefficients can be manipulated for de-noise purpose via a "hard" or "soft" thresholding (see (Daubechies, 1992) for a more general background of wavelet transform and de-noise application). Following (Haven, Liu, \& Shen, 2012), the choice of the highest decomposition level is the empirical value 7 and the lowest value is 1 . We show examples of trend and seasonal components decomposed by WT with level of 5, 4, 3 and 2 during the level selection process in Figure 2 using monthly data of tourist arrival from UK to US from Jan 1996 to Sep 2017, the same data as Figure 1. We can also clearly observe that as the level decrease, the trend component is more non-linear and leaves the seasonal component more stationary. The ADF test stops at WT with level of 3 and shows a stationary and autoregressive seasonal component in Figure 2(c).

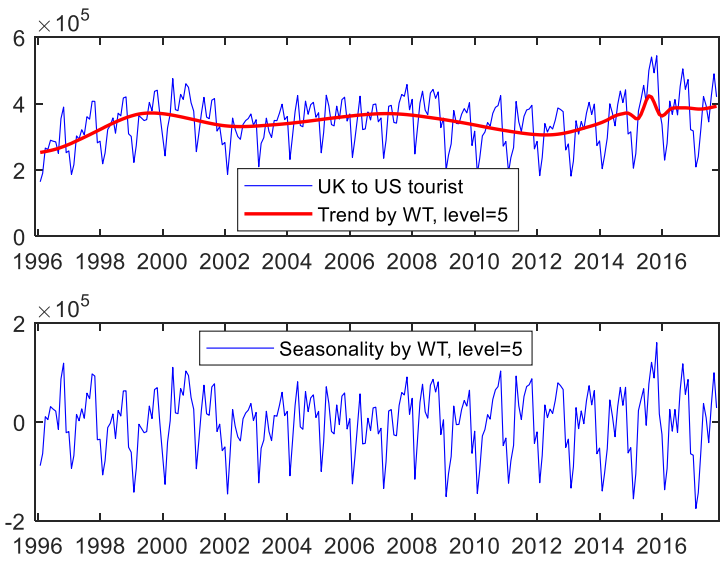

(a)
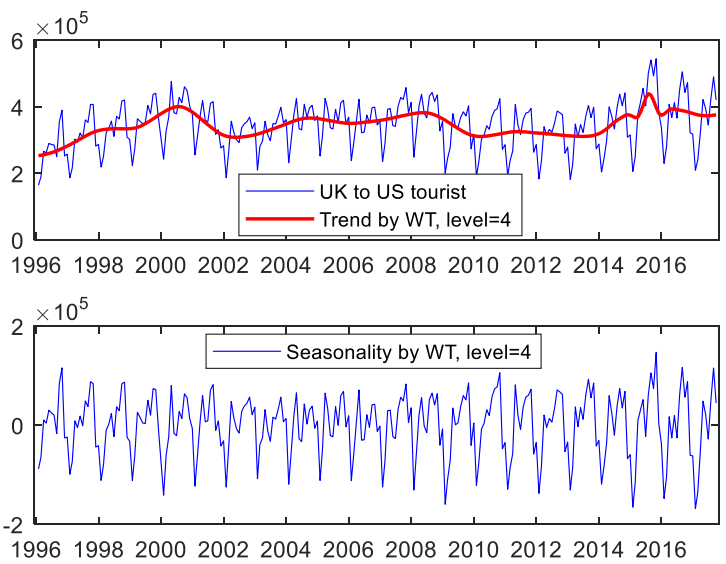

(b) 

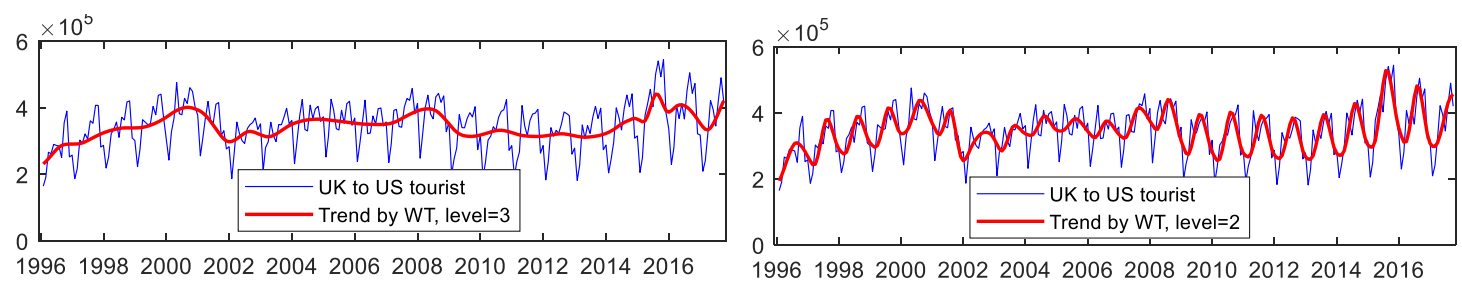

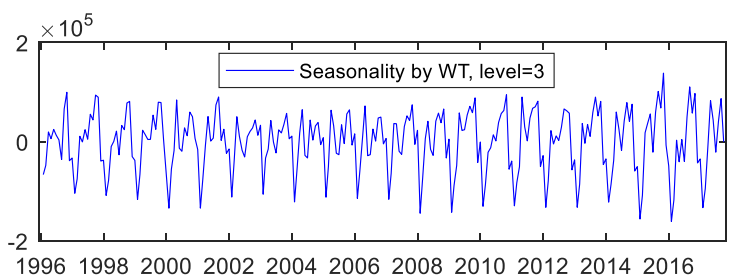

(c)

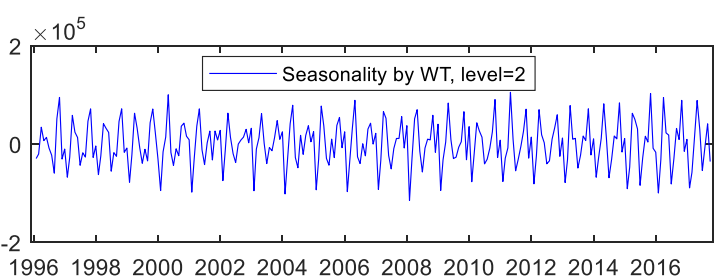

(d)

Figure 2 This figure shows the trend and seasonality decomposition by Wavelet Transformation (WT) with different levels. In upper parts of (a)-(d), blue curves represent the monthly tourist arrival from UK to US from Jan 1996 to Sep 2017 and the red curves are the trend component by WT with level 5, 4, 3 and 2 respectively. In the lower parts of (a)-(d), blue curves are the corresponding seasonal component obtain as the difference between the arrival data and the trend component respectively.

\section{Simple Moving Average (MA) method}

Simple moving average (MA) is usually used as a technical indicator in financial market. MA is considered as the equilibrium level of the equity price, which may oscillate apart or toward to the equilibrium level. In 2001, Lim et al applied MA as a method to separate the seasonal component from the tourist arrival (Lim \& McAleer, 2001) although MA may not be effective in tourism area due to the correlation between the trend and the seasonal components. Following the study of (Lim \& McAleer, 2001), we apply MA as one of the decomposition methods comparable with the HP and WT. Determining the number of lag of the MA is to obtain a seasonal component and a non-seasonal trend component. Therefore, we check the autocorrelation of the trend component and heuristically select the lag of the MA.

Since the original data $y_{t}$ shows a highly annual cyclical pattern, a lag value of $12 n, n=1,2 \ldots$ can effectively remove the cyclicality. We show an example in Figure 3 with the same data as in Figure 1. The lag value is heuristically selected as 6,9 and 12 , thus the corresponding trend components are shown in Figure 3(a), (c) and (e) respectively, and the autocorrelations of the trends are shown in Figure 3(b), (d) and (f) respectively. We can clearly observe that the repeat pattern of the trend correlations is decreasing from lag value 6 to 12 and is non-seasonal at lag of 12 . Therefore we accept the moving average with lag value 12 as the trend component in Figure 3(e). 


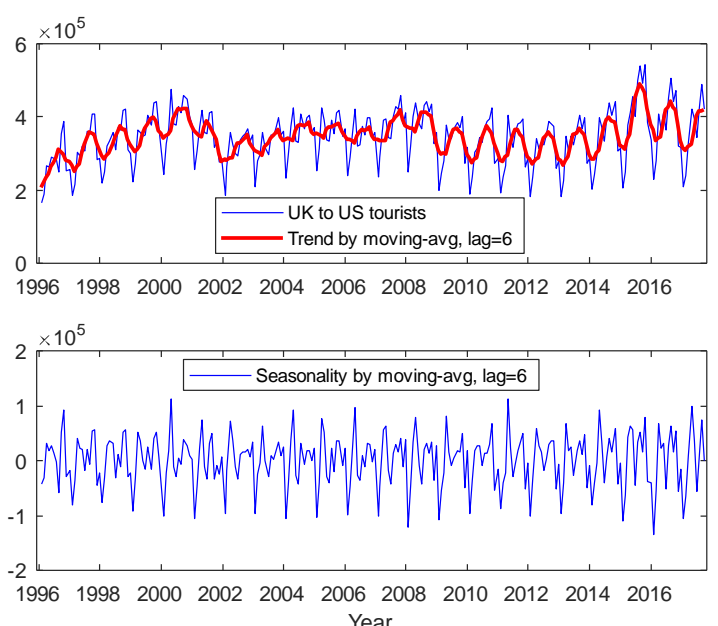

(a)
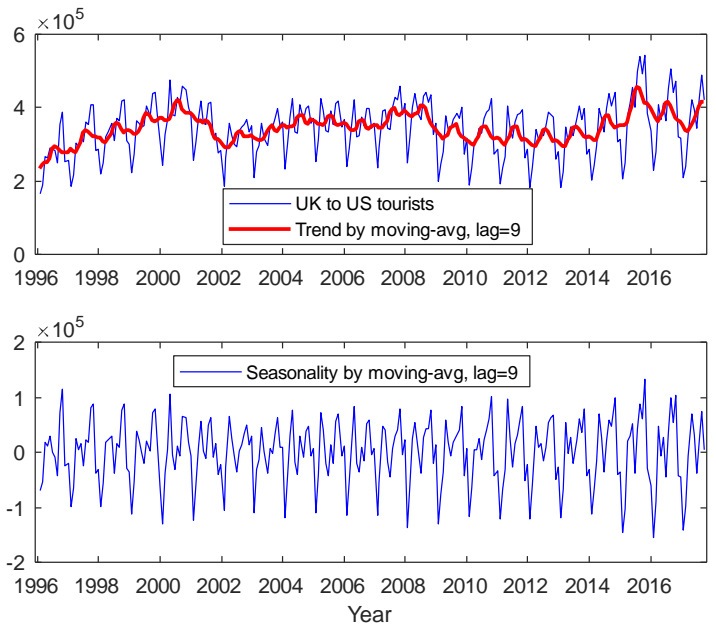

(c)
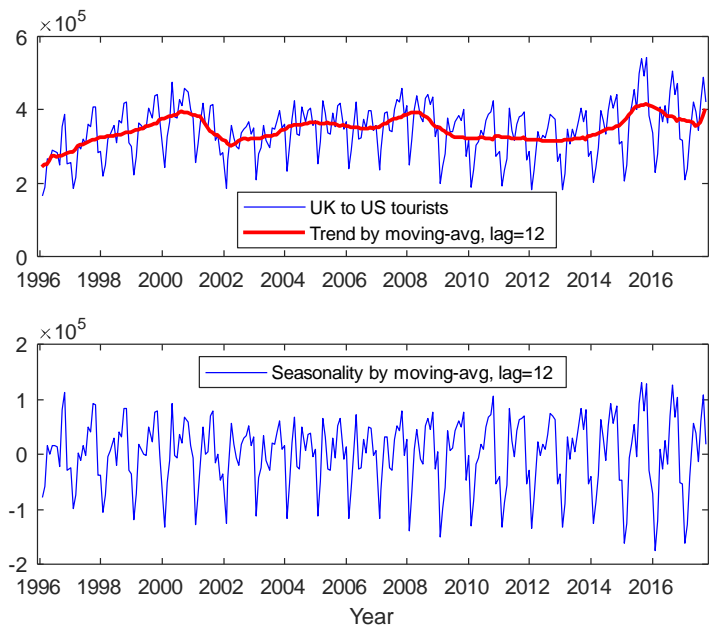

(e)

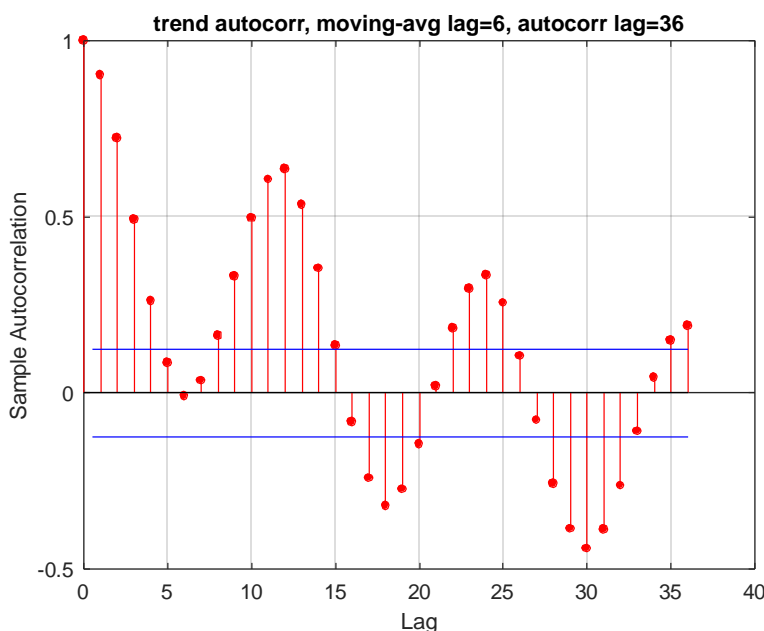

(b)

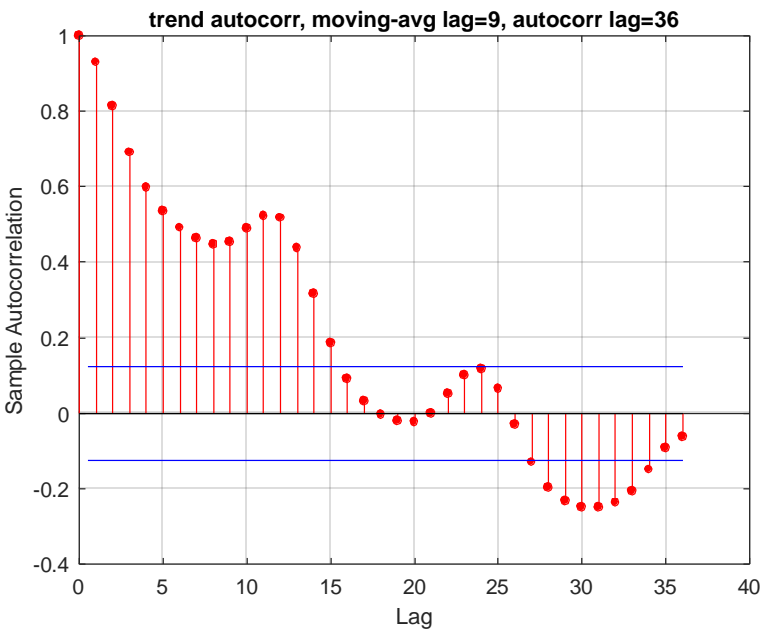

(d)

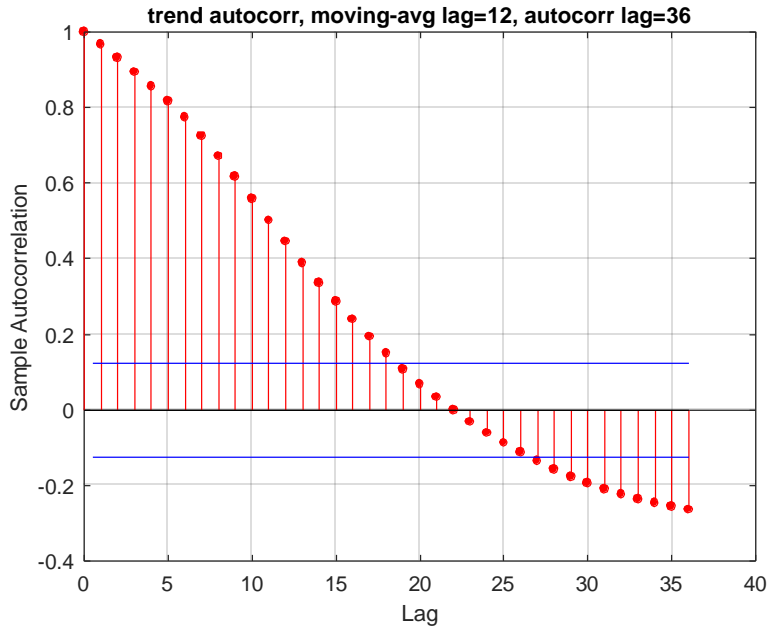

(f)

Figure 3 This figure shows a comparison of the seasonality of the trend component extracted by the moving average method with lag values 6 and 12. In upper parts of (a,c,e), blue curves represent the monthly tourist arrival from UK to US from Jan 1996 to Sep 2017 and the red curves are the trend component by moving-average (MA) with lag of 6, 9, and 12 respectively. In the lower parts of $(a, c, e)$, blue curves are the corresponding seasonal component obtain as the difference 
between the arrival data and the trend component respectively. The figures $(\mathrm{b}, \mathrm{d}, \mathrm{f})$ show the auto-correlation of the trend components in $(\mathrm{a}, \mathrm{c}, \mathrm{e})$ respectively.

We also show the autocorrelation of the seasonal component in Figure 3(e) in Figure 4. The seasonal component is extracted by MA with lag of 12 . The figure clearly shows a cyclical pattern in the seasonal component: the correlation repeats significantly at $\operatorname{lag}=12 n$, where $n=0,1, \ldots$ It means an annual repeated tourist arrival phenomenon.

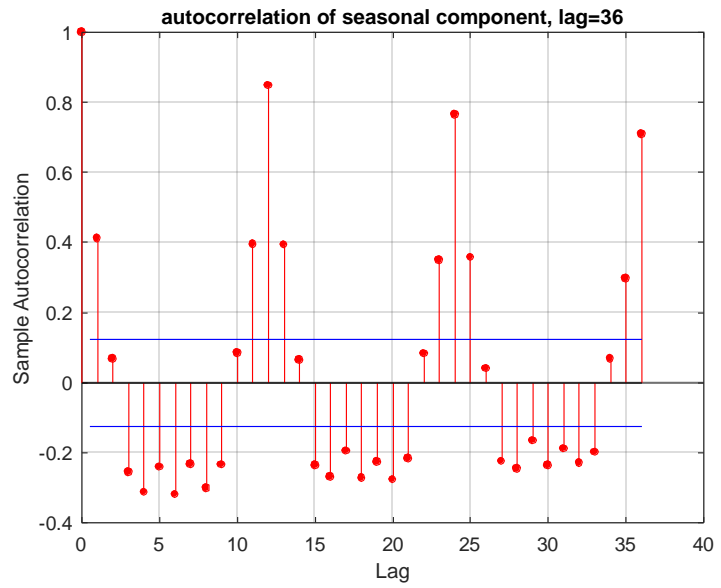

Figure 4 This figure shows the autocorrelation pattern of the seasonal component of the US tourist arrival from UK data in Jan 1996 to Sep 2017. The seasonal component is extracted by MA with lag of 12. The figure clearly shows a cyclical pattern in the seasonal component: the correlation repeats significantly at lag $=12 n$, where $n=0,1, \ldots$ It. It means an annual repeated tourist arrival phenomenon.

\subsection{Step 2: modelling the trend component}

After decomposing the trend and the seasonal component, an AutoRegressive Neural Network (ARNN) is applied to the trend component $\mu_{t}$. The ARNN is widely used in time series modelling and proved to outperform traditional models, i.e., the GARCH, EGARCH, and ARFIMA in deseasonalized financial area (Zhang \& Qi, 2005) (Patil, Tantau, \& Salokhe, 2008) (Kristjanpoller, Fadic, \& Minutolo, 2014) (Kristjanpoller \& Minutolo, 2016) and also performs better than recurrent feed-forward neural network with less sensitivity to the problem of long-term dependence (Mustafaraj, Lowry, \& Chen, 2011). In this study, to model the trend component $\mu_{t}$, we use ARNN with three layers, which include an input layer with lagged $\mu_{t}$ inputs to the network, a hidden layer with hyperbolic tangential activation function and an output layer with a linear regression function. The general form of ARNN for one-step ahead forecasts is as in (Siegelmann, Horne, \& Giles, 1997 ) and (Mustafaraj, Lowry, \& Chen, 2011):

$$
\hat{L}_{t}\left(\theta_{\mathrm{ARNN}}\right)=g\left[\varphi_{i}(t), \theta_{\mathrm{ARNN}}\right]=F_{j} \sum_{u=1}^{N_{h}} W_{j, u} f_{u}\left(\sum_{i=1}^{N_{u}} \varphi_{i}(t) w_{u, i}+w_{u}\right)+W_{j}
$$


where $g\left[\varphi_{i}(t), \theta_{\mathrm{ARNN}}\right]$ is the ARNN function; $N_{h}$ is the number of hidden neurons and $N_{u}$ is the number of input variables; $W_{j, u}$ is the weights vector from the hidden neurons to the output layers; $w_{u, i}$ represents the matrix that contains the weights from the external input $N_{u}$ to the hidden neurons $N_{h} ; w_{u}$ and $W_{j}$ are the biases of hidden and output layers; $\varphi_{i}(t)$ is the vector that contains the regression parameters of the AR part of the neural network; and $\theta_{\mathrm{ARNN}}$ specifies the parameters vector, which contains all the adjustable parameters of the neural network. In this paper, to model the trend component, we start with the widely used configuration for the ARNN following the (Siegelmann, Horne, \& Giles, 1997 ) and (Mustafaraj, Lowry, \& Chen, 2011): $N_{u}=4$, and $N_{h}=10$, which means a 4-lag input and a 10-neuron hdden layer. Therefore, the forecasting is based on current value of the $\mu_{t}$ as well as the previous 3 lags, $\mu_{t-1}, \mu_{t-2}$, and $\mu_{t-3}$. To find the best configuration, we also test the performance with $N_{h}=5,10,15$ and 20 and $N_{u}=4,6$ and 8 .

This is a supervised-learning neural network, where the model parameters are "trained" to map the input-output variables via the modified Levenberg-Marquardt (Hagan \& Menhaj., 1994) algorithm, which is to minimize the quadratic error by descent of the maximum gradient. The following Figure 5 shows two examples of forecasting the trend component by trained ARNN using the same data as Figure 1. In Figure 5(a), the trend component is extracted by HP filter with $\lambda=100$ and in Figure 5(b), the trend component is obtained by 12 lag moving average. The trend component data from Jan 1996 to Jul 2013 in (a) and (b) are used to train the ARNN model separately and the remained data from Aug 2013 to Sep 2017 is used for out-of-sample testing the two ARNN models. In the upper part of Figure 5(a), the forecasted (red) and the extracted trend components are illustrated and show a very closed pattern. The middle and lower parts of Figure 5(a) and (b) show the absolute error and absolute percentage error of the two forecasted trends respectively. We observe very competitive performances of below $1 \%$ error in (a) and below $2 \%$ error in (b) by ARNN, although the error in Figure 5(b) is higher than (a) due to the non-smoothness of the trend by moving average. 

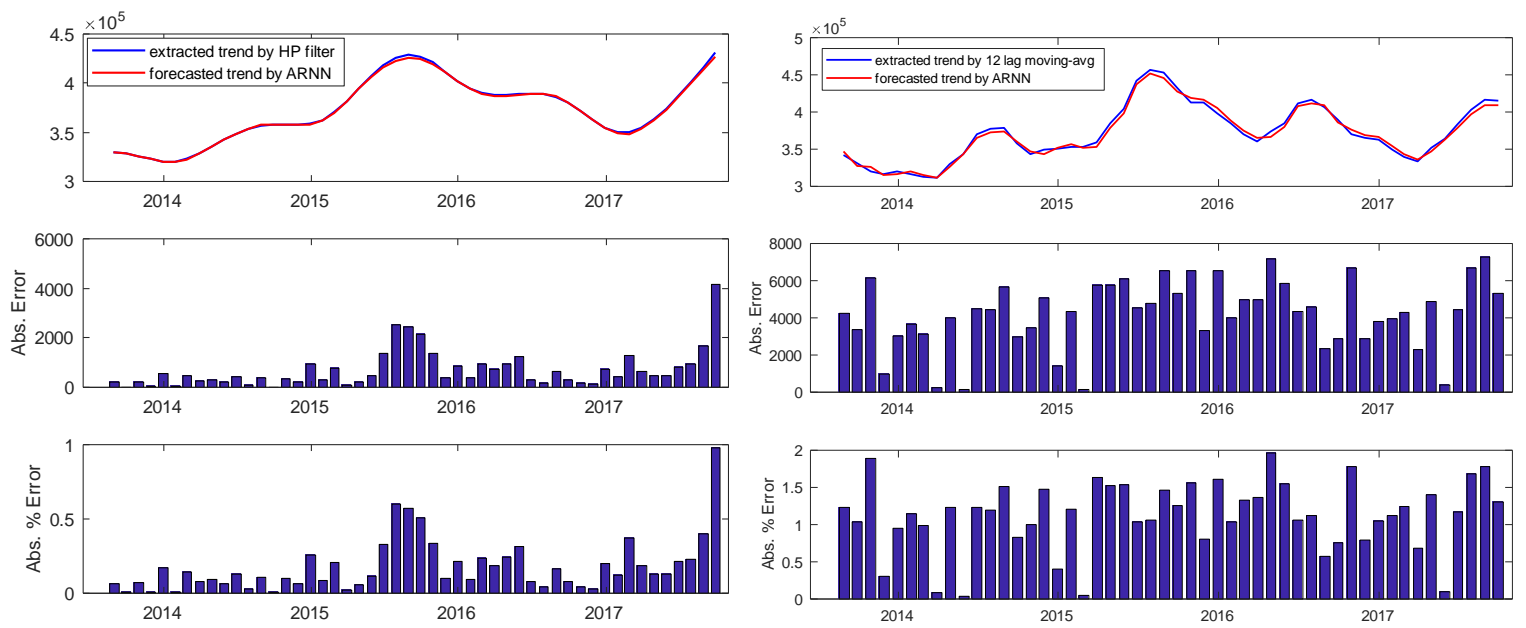

(a)

(b)

Figure 5 This figure shows an example of forecasting the trend component only by ARNN model. (a) in the upper figure, the blue curve is the trend component extracted from the monthly tourist arrival from UK to US from Aug 2013 to Sep 2017 via the HP filter with $\lambda=\mathbf{1 0 0}$. The red curve is the forecasted trend by ARNN, which is trained by the trend component data from Jan 1996 to Jul 2013. The middle and lower figures show the absolute error and absolute percentage error of the forecasted trend respectively; (b) in the upper figure, the blue curve is the trend component by the 12 lag moving average of the same data. The red curve the forecasted trend by ARNN. The middle and lower figures show the absolute error and absolute percentage error of the forecasted trend respectively.

After modelling the trend component, we model the seasonal component by a separated ARNN model. (Song, Smeral, G. Li, \& Chen, 2013) found that a four-quarter lag time-varying parameter model can capture seasonal patterns well in forecasting the quarterly tourist arrival series. Inspired by this study, we simply extend the lag to 12-month to forecast the monthly tourist arrival series in our $p$ NN models. This also complies with the autocorrelation test in Figure 4, where the seasonal component shows a clear annual cycle at lag $=12 n$, where $n=0,1 \ldots$ Therefore, setting the number of the seasons in a year $s$ as 12 reflects the nature of the data and complies with the literature.

\subsection{Step 3: $n$-step ahead forecasting}

Finally, the $n$-step ahead forecast of the monthly tourist arrivals is the sum of the output from two separated ARNN models according to the deterministic equation (1): $\hat{y}_{t+n}=\hat{\mu}_{t+n}+\hat{\gamma}_{t+n}$, where $\hat{\mu}_{t+n}$ and $\hat{\gamma}_{t+n}$ are the $n$-step trend and seasonal component forecasting results generated by two separated ARNN models respectively.

\subsection{Flow of the methodology}

We illustrate the implementation of a pair of Neural Network $(p N N)$ in the following Figure 6. In this figure, the key steps of implementing the $p \mathrm{NN}$, the decomposition of the trend component, testing 
of the stationarity of the seasonal component and the two separated autoregressive neural networks are shown with the data flow diagram.

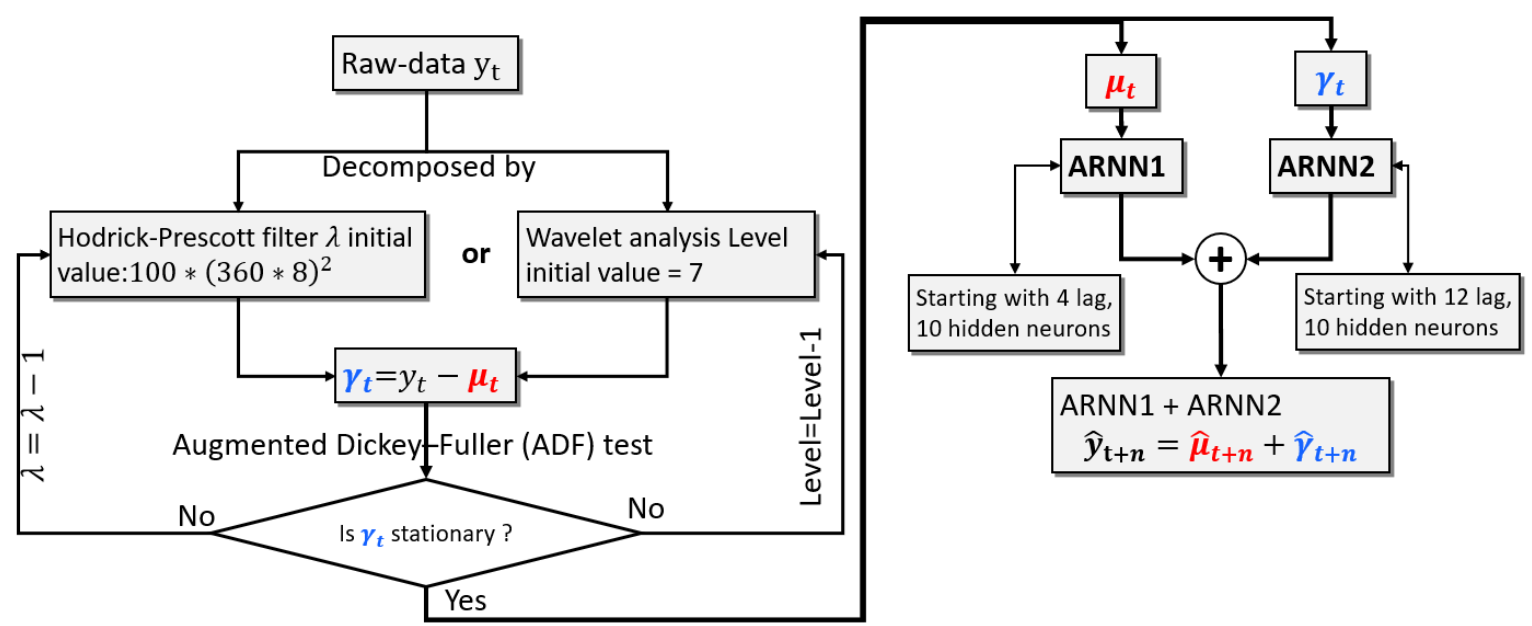

Figure 6 This figure shows the implementation flow of the pair of autoregressive neural network $(p N N)$ method

\section{Forecasting evaluation}

\subsection{Data and $p N N$ configurations}

The empirical study of the propose $p \mathrm{NN}$ is based on US monthly inbound tourist arrivals from top 12 source markets, including Mexico, Canada, China Mainland, Japan, United Kingdom, South Korea, Brazil, Germany, Australia, France Italy and Spain in the last 22 years (from Jan 1996 to Sep 2017). The time series data for each source market are downloaded from the official website of National Travel \& Tourism Office. The Figure 7 shows an illustration of the inbound tourist arrival data series of the top 12 source markets from Jan 1996 to Sep 2017. It is obviously that over the last six years from 2010 to 2016, tourist arrivals from Mexico and China Mainland have a jump increase. Note that the significantly seasonal pattern is a normal feature of US inbound tourist arrival across all source market. 


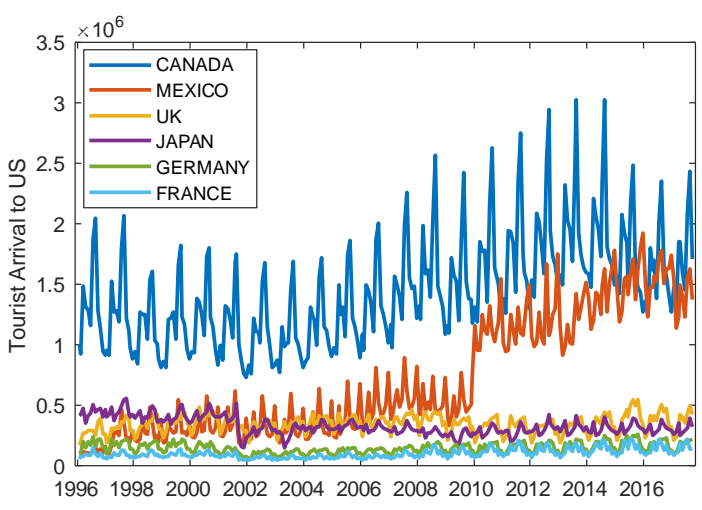

(a)

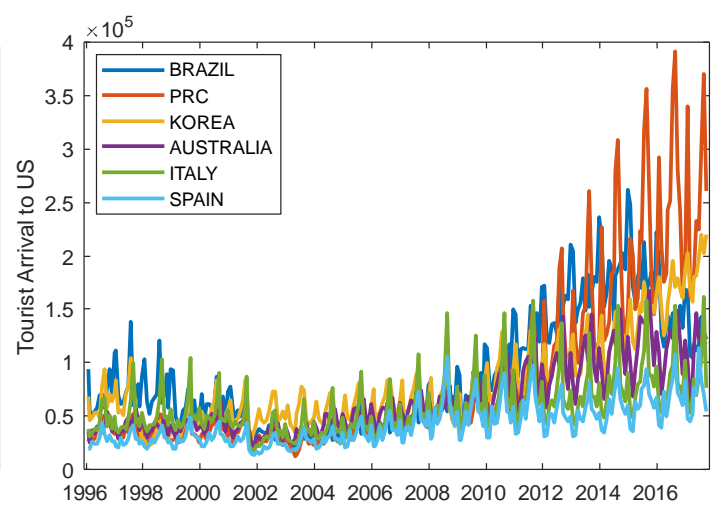

(b)

Figure 7 This figure shows all tourist arrival data to US from Jan 1996 to Sep 2017; (a) the tourist arrival from top 6 source markets: Canada, Mexico, UK, Japan, Germany, France; (b) the tourist arrival from the $7^{\text {th }}-12^{\text {th }}$ source markets: Brazil, China Mainland, South Korea, Australia, Italy, and Spain.

To obtain the best forecasting result, we compare the performances of the whole $p \mathrm{NN}$ under different configurations of the two ARNN models. We summarize the configurations in Table 1.

Table 1 This table summarizes the configurations of a pair of ARNN models for trend and seasonal components respectively.

\begin{tabular}{llll}
\hline & Input lag $N_{u}$ & Hidden neuron $N_{h}$ & Training length \\
\hline ARNN trend & $4,8,12$ & $5,10,15,20$ & From 2 years to 19.5 years with step of 0.25 year \\
\cline { 2 - 4 } ARNN seasonal & 12 & & \\
\hline
\end{tabular}

Therefore the $p$ NN performance evaluation is composed of two parts:

1) Part 1-Performance study under different configurations: we construct $p N N$ model under the configurations in Table 1 and compare the performances to obtain an appropriate configuration for $p \mathrm{NN}$;

2) Part 2-Performance comparison: we construct $p N N$ with HP, WT, and MA under the selected configuration and compare the performance with the benchmark models.

\subsection{Benchmark Models}

The $p$ NN models are constructed by three steps in Section 2 with the low-pass Hodrick-Prescott (HP) filter, wavelet transformation (WT), and the moving-average (MA) and are named as $p \mathrm{NN}-\mathrm{HP}, p \mathrm{NN}$ $\mathrm{WT}$, and $p \mathrm{NN}-\mathrm{MA}$. As the comparison, selected traditional econometrics models are also included as the benchmark. The Seasonal Autoregressive Integrated Moving Average (SARIMA) model assumes that the tourist arrival data is also composed of trend and seasonal components as well as irregular terms. It stabilizes the time series by seasonal and non-seasonal differencing, which is widely applied in financial area for reducing the non-stationarity. As the SARIMA model is almost 
the most widely applied model in tourism demand forecasting, and performs reasonably well (Alleyne, 2006) (Kulendran \& Wong, 2005) (Oh \& Morzuch, 2005), it is selected as one of the benchmark models with other two traditional models, autoregressive integrated moving average (ARIMA) and autoregressive fractionally integrated moving average (ARFIMA) model.

For the first part of the evaluation, we select the $p$ NN-HP, $p$ NN-WT, and $p$ NN-MA as the example to test the performances of the model using different length of training data. The training dataset is selected from 2 years up to 19.5 years. For each length, the $p$ NN-HP, $p$ NN-WT, and $p$ NN-MA model is trained and then tested at horizon of 1 month to 18 months (1.5 years).

For the second part, we employ a rolling-window mechanism to evaluate the forecasting performance. The data in the rolling-window is used to estimate the models and the remained following-up data is used as the testing dataset. The initial window, W1, is set to from Jan 1996 to Mar 2016 and the remained data of 18 months, from Apr 2016 to Sep 2017 are set to the testing dataset. The models are, firstly, estimated by the data in $W 1$, and then, tested by the remained data of 18 months for a set of horizon $h$ of 1 to 18 months ahead forecasts. After this, the initial window slides one month forward to W2, covering from Feb 1996 to Apr 2016, for estimating the models, and the remained data of 17 months, from May 2016 to Sep 2017 is for testing the models. In this round, a set of horizon $h$ of 1 to 17 months ahead forecasting results are generated and tested. Similarly, the model estimation and forecasting are recursively repeated until all remained data are used up. In the end, the rolling-window forecast mechanism generates $19-h$ sets of $h(h=1, \ldots, 18)$ months ahead forecasting results, which is, 2052 in total (12 source markets and 171 forecasts in each market).

The forecast accuracy is evaluated by the Mean Average Absolute Percentage Error (MAPE) as it is a relative measure and comparable across different datasets. In addition, the forecasted values of the tourist arrivals are also shown as examples for a closer insight.

\subsection{Empirical Result}

\subsubsection{Part 1: Performance study under different configurations}

Training length 
To find out an optimal length of training dataset for all models, we train $p \mathrm{NN}$ models with 4 input lags and 10 hidden neurons. Such configuration is merely for the length determination. Thus, we train $p$ NN-HP, $p$ NN-WT, and $p$ NN-MA as well as ARIMA and ARFIMA models by twelve datasets with length from 2 to 20 years. We test the trained models by forecasting the values at 1 to 18 months (1.5 years) horizons. The MAPE performance of the $p$ NN-HP, $p$ NN-WT, and $p$ NN-MA against different length of training dataset are shown in Figure 8(a). As the $p$ NN-HP, $p$ NN-WT, and $p$ NNMA show relatively better performances at training dataset longer than 200 months (16.7 years), to obtain a generic length of the dataset, we calculate the average MAPE of the three models across twelve datasets in Figure 9. We can clearly observe that training the models by the data of 210 (17.5 years) months achieves the best generic performance. Similarly, the benchmark models, ARIMA and ARFIMA, are estimated and tested as the same method. The average MAPE of ARIMA and ARFIMA across all datasets are shown in Figure 8(b) (The MAPE of ARIMA and ARFIMA on each single dataset is shown in appendix). As we can also clearly observe that with the training dataset of 210 months, those two models achieve the best performance, although the overall performance is around 50\% and is significantly lower than the one in Figure 8(a), which is around $2 \%$.
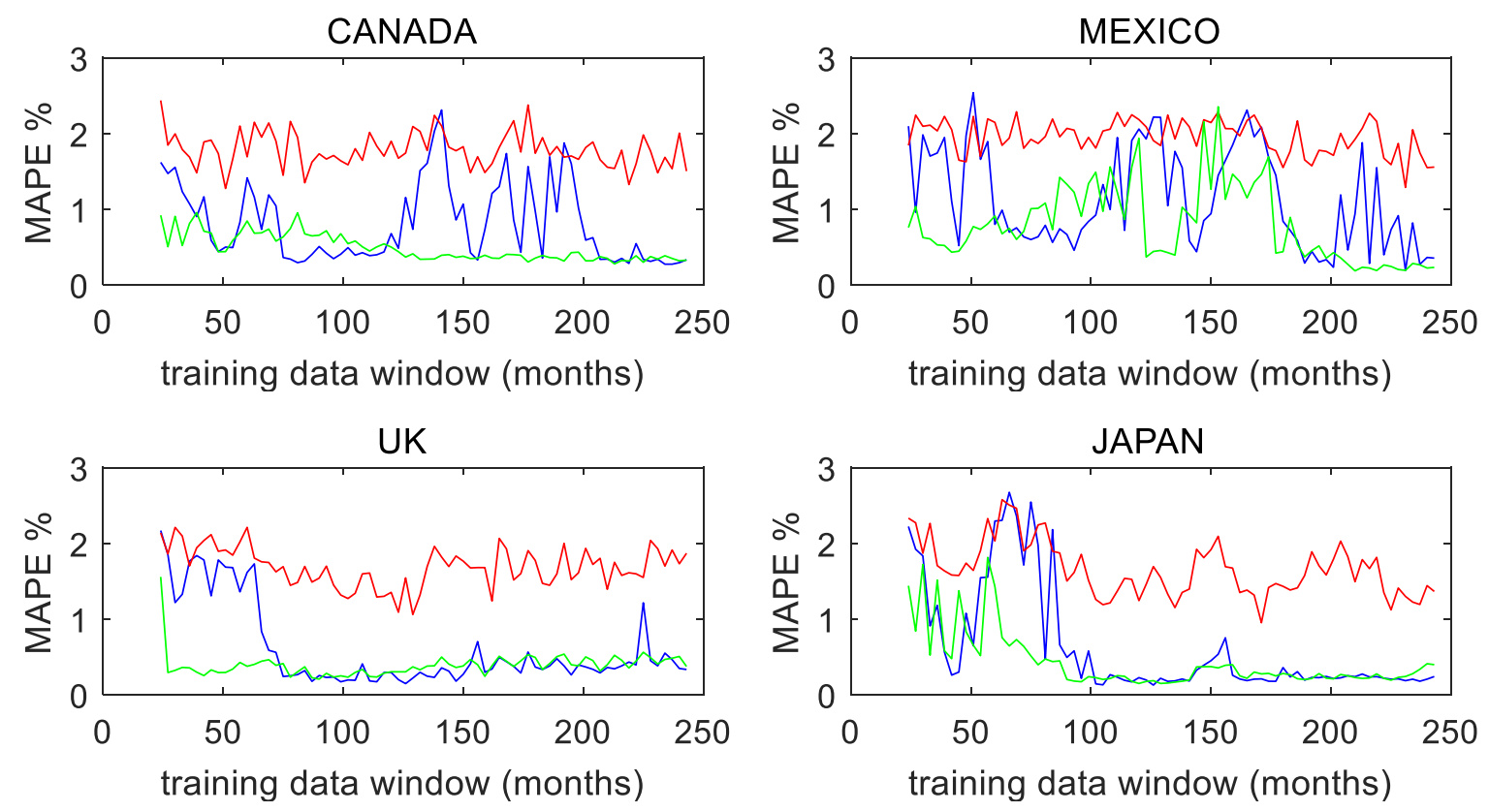

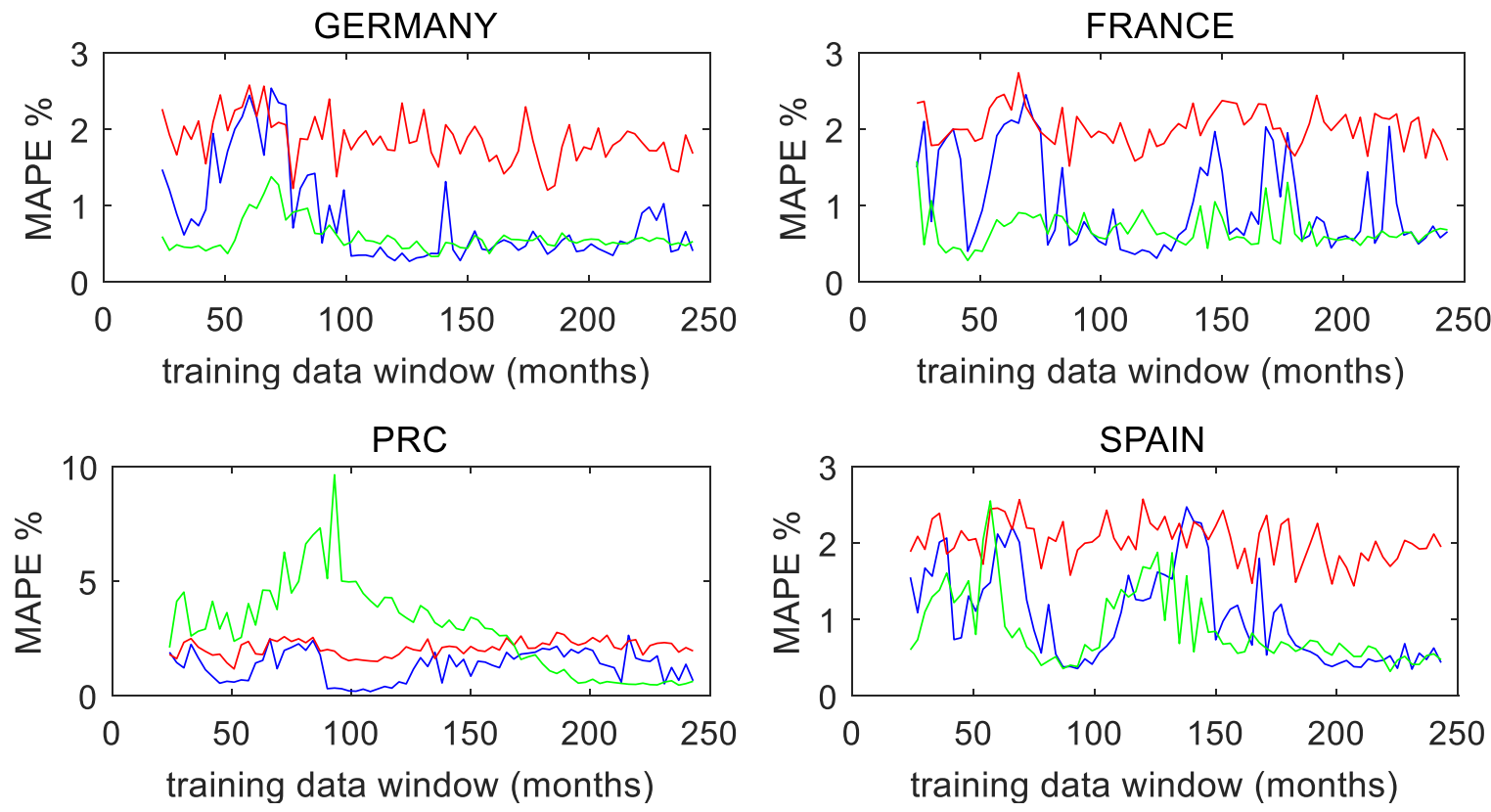

ITALY
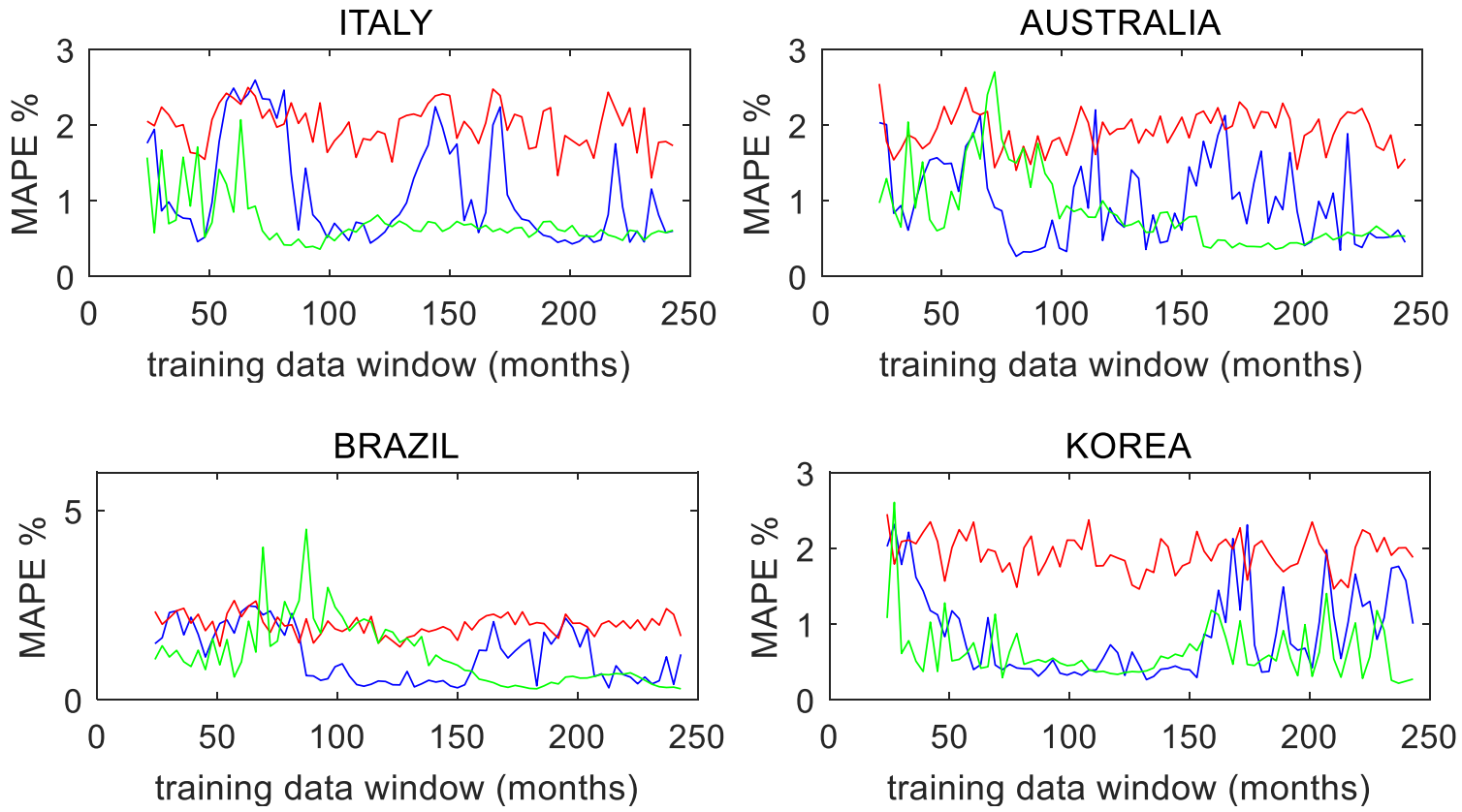

$-\mathrm{pNN}-\mathrm{HP} \longrightarrow \mathrm{pNN}-\mathrm{MA}-\mathrm{pNT}$

Figure 8 This figure shows the performance (MAPE) of the $p$ NN-HP, $p$ NN-MA, and $p$ NN-WT by the training dataset with length from 2 to 20 years. The evaluation is based on tourist arrival data to US from top 12 source markets: Canada, Mexico, UK, Japan, Germany, France, China, Spain, Italy, Australia, Brazil and Korea in the time period from Jan 1996 to Sep 2017. 


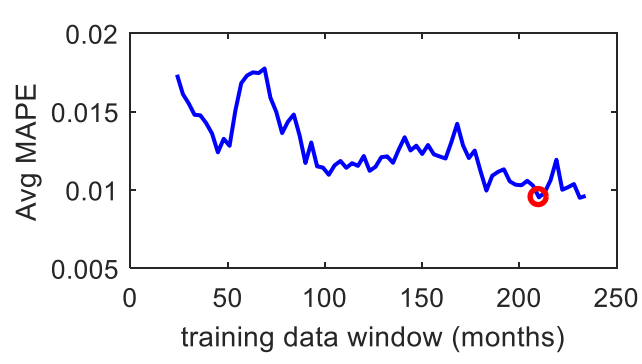

(a)

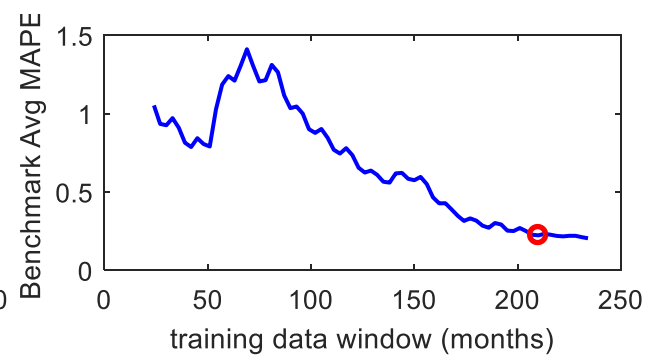

(b)

Figure 9 This figure shows the average MAPE of (a) the $p$ NN-HP, $p$ NN-MA, and $p$ NN-WT; and (b) ARIMA, and ARFIMA across all datasets in Figure 8. The red circle indicates the best generic performance. The length of the training dataset at best-performed point is 210 months, equivalent to 17.5 years.

Tourism industry is not a data-intensive area as the tourist arrival is usually recorded monthly, which means that training a model using the data of 17.5 years and forecasting 1.5 years ahead is merely equivalent to forecasting 18 data samples ahead based on 210 historical data samples. Such a configuration has been proved by the empirical studies on twelve datasets as the best one in terms of the forecasting performance.

\section{$\underline{\text { Hidden Neurons }}$}

After determining the length of the training dataset, we tune the number of hidden neurons in $p$ NN models to obtain an optimal parameter. We train the $p \mathrm{NN}-\mathrm{HP}, p \mathrm{NN}-\mathrm{WT}$, and $p \mathrm{NN}-\mathrm{MA}$ models using the data of 17.5 years with 5,10,15 and 20 hidden neurons and show the 1 to 18 months (1.5 years) forecasting performance by MAPE. We show the MAPE performance of $p \mathrm{NN}-\mathrm{HP}, p \mathrm{NN}-\mathrm{WT}$, and $p$ NN-MA models on four examples, tourist arrival from Canada, Mexico, UK and Japan. The average MAPE across all forecasting horizons are shown in the Figure 10. To illustrate an overall performance, we calculate the average MAPE of $p \mathrm{NN}-\mathrm{HP}, p \mathrm{NN}-\mathrm{WT}$, and $p \mathrm{NN}-\mathrm{MA}$ models across all horizons and show then in the Table 2. It is quite clear that in all datasets except Korea, the $h \mathrm{NN}$ model with 5 hidden neurons achieves the lowest average MAPE. We therefore follow the empirical study and train the $h \mathrm{NN}$ models with five hidden neurons.

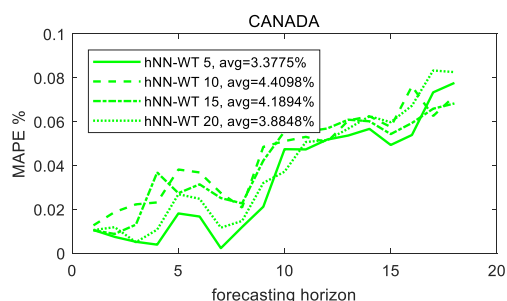

(a)

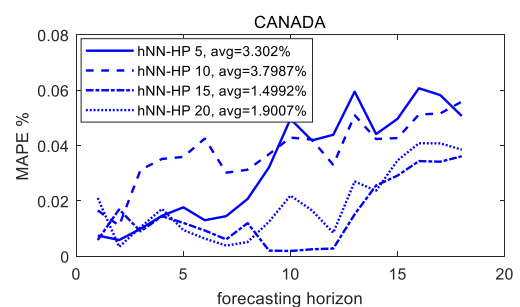

(b)

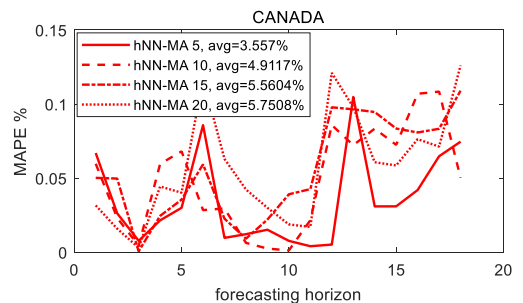

(c) 


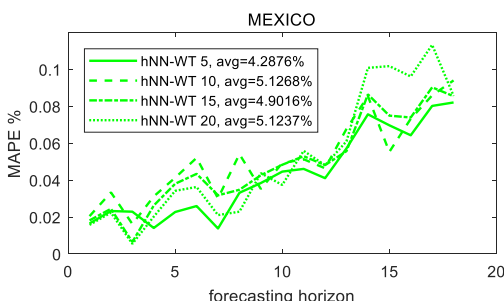

(d)

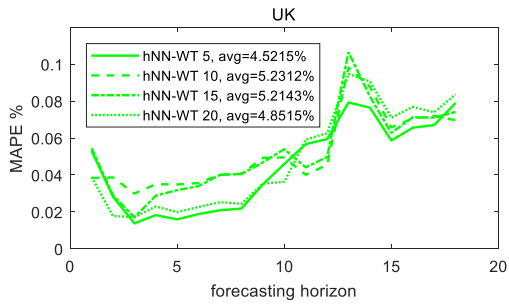

(g)

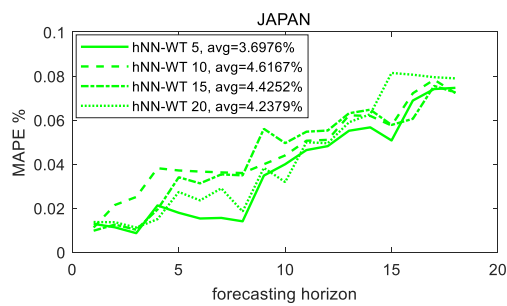

(j)

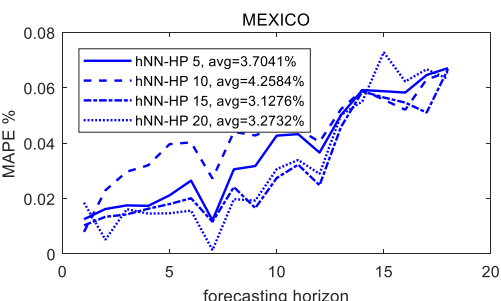

(e)

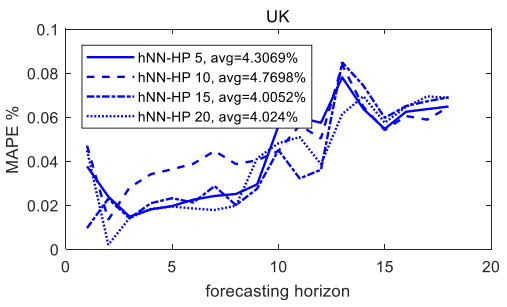

(h)

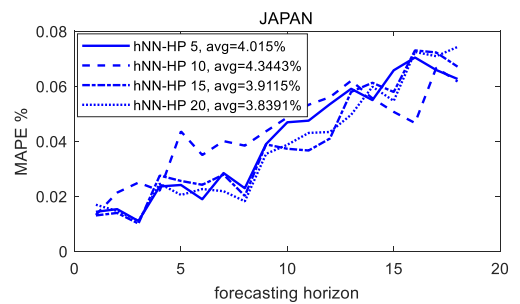

(k)

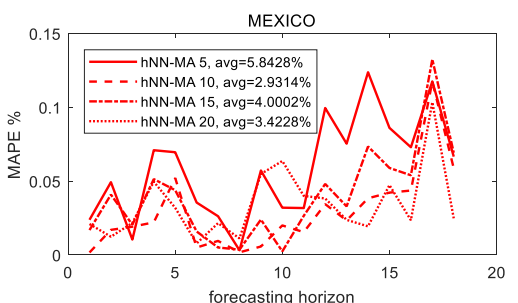

(f)

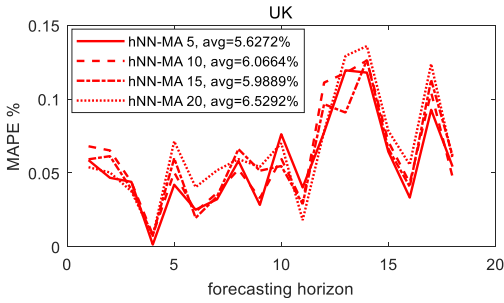

(i)

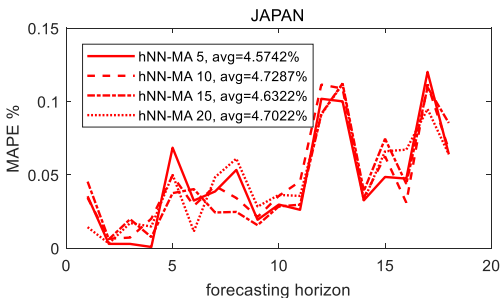

(1)

Figure 10 This figure shows four example datasets of the testing MAPE of $p$ NN-HP, $p$ NN-WT, and $p$ NN-MA models trained with 5, 10, 15, and 20 neurons.

Table 2 This table shows the average MAPE of three models, $p$ NN-HP, $p$ NN-WT, and $p$ NN-MA, over all forecasting horizons under the configuration of $5,10,15$, and 20 hidden neurons.

\begin{tabular}{|c|c|c|c|c|c|c|}
\hline \multicolumn{6}{|c|}{ CANAD } & \multirow{2}{*}{$\begin{array}{c}\text { KORE } \\
\text { A } \\
\end{array}$} \\
\hline hidden neuron & MEXICO & $\mathrm{A}$ & PRC & JAPAN & UK & \\
\hline \multirow{3}{*}{ 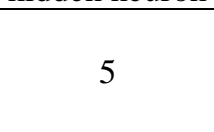 } & & 3.4122 & & 4.0956 & 4.8185 & 3.2335 \\
\hline & $3.9399 \%$ & $\%$ & $2.4468 \%$ & $\%$ & $\%$ & $\%$ \\
\hline & & & & 4.5632 & 5.3558 & 3.4572 \\
\hline \multirow[t]{2}{*}{10} & $4.1055 \%$ & $4.3734 \%$ & $4.1284 \%$ & $\%$ & $\%$ & $\%$ \\
\hline & & & & 4.3230 & 5.0695 & 2.6726 \\
\hline \multirow[t]{2}{*}{15} & $4.0098 \%$ & $3.7497 \%$ & $4.9345 \%$ & $\%$ & $\%$ & $\%$ \\
\hline & & & & 4.2597 & 5.1349 & 2.6066 \\
\hline \multirow[t]{2}{*}{20} & $4.6115 \%$ & $3.8454 \%$ & $2.6438 \%$ & $\%$ & $\%$ & $\%$ \\
\hline & & GERMA & AUSTRA & FRANC & & \\
\hline \multirow[t]{2}{*}{ hidden neuron } & BRAZIL & NY & LIA & E & ITALY & SPAIN \\
\hline & & 4.0641 & & 4.6791 & 4.6849 & 4.7292 \\
\hline \multirow[t]{2}{*}{5} & $3.1354 \%$ & $\%$ & $3.3082 \%$ & $\%$ & $\%$ & $\%$ \\
\hline & & & & 5.2291 & 5.4034 & 4.9348 \\
\hline \multirow[t]{2}{*}{10} & $5.2411 \%$ & $5.0720 \%$ & $3.3496 \%$ & $\%$ & $\%$ & $\%$ \\
\hline & & & & 5.2152 & 4.9562 & 5.1372 \\
\hline \multirow[t]{2}{*}{15} & $4.6445 \%$ & $4.6191 \%$ & $4.7678 \%$ & $\%$ & $\%$ & $\%$ \\
\hline & & & & 5.1556 & 4.9787 & 5.1303 \\
\hline 20 & $4.8961 \%$ & $4.9767 \%$ & $2.7879 \%$ & $\%$ & $\%$ & $\%$ \\
\hline
\end{tabular}

$\underline{\text { Input lags }}$ 
In previous empirical studies of determining the training dataset length and hidden neurons, the input lags of trend and seasonal component are selected as 4 and 12 respectively. As the autocorrelation study in Figure 4 shows that the seasonal component is strongly autocorrelated of lag 12, we select 12 as the lag value for the seasonal component. For the trend component, we follow the configurations (5 neurons and training length 210 months) in previous discussion and study the performances of $p \mathrm{NN}$ models with 4, 8, and 12 input lags in Table 1. The performance comparison in the following Table 3 shows that the $p$ NN models with input lag of 4 outperforms all other lag values consistently across all datasets.

Table 3 This table shows the average MAPE of three models, $p$ NN-HP, $p$ NN-WT, and $p$ NN-MA, over all forecasting horizons under the configuration of 4,8 , and 12 input lags for trend component ARNN

\begin{tabular}{ccccccc}
\hline input lag & MEXICO & CANADA & PRC & JAPAN & UK & KOREA \\
\hline 4 & $\mathbf{3 . 9 3 9 9 \%}$ & $\mathbf{3 . 4 1 2 2 \%}$ & $\mathbf{2 . 6 4 3 8 \%}$ & $\mathbf{4 . 0 9 5 6 \%}$ & $\mathbf{4 . 8 1 8 5 \%}$ & $\mathbf{3 . 2 3 3 5 \%}$ \\
8 & $4.1475 \%$ & $4.4152 \%$ & $4.1380 \%$ & $4.5642 \%$ & $5.4324 \%$ & $3.4675 \%$ \\
12 & $4.0153 \%$ & $3.8022 \%$ & $4.9850 \%$ & $4.3492 \%$ & $5.0979 \%$ & $5.2058 \%$ \\
\hline & BRAZIL & GERMANY & AUSTRALIA & FRANCE & ITALY & SPAIN \\
\hline 4 & $\mathbf{3 . 1 3 5 4 \%}$ & $\mathbf{4 . 0 6 4 1 \%}$ & $\mathbf{3 . 3 0 8 2} \%$ & $\mathbf{4 . 6 7 9 1 \%}$ & $\mathbf{4 . 6 8 4 9 \%}$ & $\mathbf{4 . 7 2 9 2 \%}$ \\
8 & $5.3028 \%$ & $5.1131 \%$ & $3.4046 \%$ & $5.2797 \%$ & $5.4464 \%$ & $4.9352 \%$ \\
12 & $4.7021 \%$ & $4.9972 \%$ & $4.7809 \%$ & $5.3087 \%$ & $4.9657 \%$ & $5.2088 \%$ \\
\hline
\end{tabular}

To summarize the empirical studies of the Part 1 evaluation, we have the $p$ NN configuration as: 4 lags ARNN for trend component, 12 lags ARNN for seasonal component and the training dataset length of 210 months.

\subsubsection{Part 2: Performance comparison}

In this part, we follow the configurations of $p \mathrm{NN}-\mathrm{HP}, p \mathrm{NN}-\mathrm{WT}$, and $p \mathrm{NN}-\mathrm{MA}$ models discussed in Section 3.3.1. We also evaluate the performances under different parameters heuristically as the traditional application. In $p$ NN-HP model, the penalty parameter $\lambda$ is selected as $100,1600,3600$, and 129600, where the value of 100 is also the selection result by our method in Step 1 in Section 2.1. In the $p \mathrm{NN}-\mathrm{WT}$ model, the wavelet transformation level is selected as $3,4,5$, and 6 , where level 3 is the selection result by our method. For $p$ NN-MA, we only follow the configuration of MA with lag of 12 as the Figure 3(e,f) due to the discussion in Section 2.1.

The evaluation has two parts. Firstly, we estimate the $p$ NN-HP, $p$ NN-WT, and $p$ NN-MA with the benchmark models ARIMA, ARFIMA, and SARIMA with the fixed length (210 months) of training 
dataset and test the forecasting performance of horizon from 1 to 18 months. In the second part, we estimate the models by the same length (210 months) of training dataset and evaluate the forecasting results, afterwards, we slide forward a window of 210 months by one-month step ahead and evaluate the forecasting results again. The training window will be slid until the testing horizon reaches the end of the dataset. This sliding window evaluation is not for enhancing the model performance but merely evaluate the model in a pseudo-practical context and compare the average performances across certain period. Those two parts cover a complete spectrum of the evaluation and show us a stable comparison result.

\section{$\underline{\text { Part } 1 \text { results }}$}

The following Table 4 to Table 15 show the first part experiments results of the US tourist arrival from six source countries, Japan, P.R.China, Canada, Mexico, France and UK. The other six datasets (Korea, Brazil, Germany Australia, Italy, and Spain) are shown in the appendix. The performances of $p \mathrm{NN}-\mathrm{HP}, p \mathrm{NN}-\mathrm{WT}$ and $p \mathrm{NN}-\mathrm{MA}$ models with different parameters as well as ARIMA, ARFIMA, and SARIMA models are compared from horizon 1 to 18 months. In addition, the average performance over 18 horizons are also calculated and compared. As we observed from Table 4, Table 6 , Table 8 , Table 10, Table 12 and Table 14, pNN-HP with $\lambda=100$ achieves the best average MAPE compared with $\lambda=100,1600,3600$ and 129600 and $p$ NN-WT with level 3 achieves the best average MAPE compared with other levels. This observation is consistent in all six datasets as well as the ones in the appendix. It shows the effectiveness of our proposed approach in Section 2. Therefore, an explicit decomposition with a stationary seasonal component and an unspecified non-linear trend component enhances the forecasting performance than the traditional heuristic selection of the decomposition parameters.

Among the pNN family models, the pNN-HP achieves slightly better performance than the pNNWT in 12 datasets, where pNN-HP reaches a lower average MAPE than pNN-WT in eight datasets of Japan, P.R.China, Canada, UK, Korea, Brazil, Germany, and Australia while pNN-WT outperforms the pNN-HP in other four datasets. Both $\mathrm{pNN}-\mathrm{HP}$ and $\mathrm{pNN}-\mathrm{WT}$ reach a better performance than pNN-MA in all datasets. Similarly, in the Table 5, Table 7, Table 9, Table 11, Table 13, and Table 15, the SARIMA model outperforms the ARIMA and ARFIMA models consistently across all datasets. In most of the cases, SARIMA model achieves the average MAPE of 5\% while the ARIMA and ARFIMA models achieve the average MAPE higher than $9 \%$. 
Table 4 This table shows the accuracy of monthly forecasts of US tourist arrivals from Japan. The Absolute Percentage Error (APE) is calculated at forecasting horizon 1 to 18 months (1.5 years). The $p \mathrm{NN}-\mathrm{HP}, p \mathrm{NN}-\mathrm{WT}$ and $p \mathrm{NN}-\mathrm{MA}$ models are all estimated by the data from Jan 1996 to Jun 2013 (210 months).

\begin{tabular}{|c|c|c|c|c|c|c|c|c|c|}
\hline \multirow[b]{2}{*}{ horizon } & \multicolumn{4}{|c|}{$p$ NN HP } & \multicolumn{4}{|c|}{$p$ NN WT } & \multirow{2}{*}{$p$ NN MA } \\
\hline & 100 & 1600 & 3600 & 129600 & 3 & 4 & 5 & 6 & \\
\hline 1 & $1.2946 \%$ & $1.3299 \%$ & $1.2111 \%$ & $1.9526 \%$ & $0.9437 \%$ & $1.4837 \%$ & $1.3342 \%$ & $0.9319 \%$ & $4.5265 \%$ \\
\hline 2 & $1.3851 \%$ & $1.4071 \%$ & $1.4177 \%$ & $1.2317 \%$ & $1.7877 \%$ & $1.3717 \%$ & $1.3479 \%$ & $1.4417 \%$ & $0.5969 \%$ \\
\hline 3 & $0.9988 \%$ & $1.7971 \%$ & $2.2607 \%$ & $1.8639 \%$ & $0.8011 \%$ & $1.9406 \%$ & $1.0041 \%$ & $0.8373 \%$ & $1.9668 \%$ \\
\hline 4 & $2.7431 \%$ & $2.8666 \%$ & $2.9668 \%$ & $2.7712 \%$ & $0.9987 \%$ & $2.7956 \%$ & $2.5190 \%$ & $2.5525 \%$ & $0.7449 \%$ \\
\hline 5 & $2.5481 \%$ & $2.5707 \%$ & $2.8107 \%$ & $2.5184 \%$ & $2.6446 \%$ & $2.8578 \%$ & $2.6305 \%$ & $2.7294 \%$ & $3.7428 \%$ \\
\hline 6 & $2.4086 \%$ & $2.3654 \%$ & $2.4513 \%$ & $2.0173 \%$ & $2.6171 \%$ & $1.7597 \%$ & $1.8700 \%$ & $2.1986 \%$ & $4.0164 \%$ \\
\hline 7 & $2.7795 \%$ & $2.8217 \%$ & $2.8956 \%$ & $2.0977 \%$ & $2.5873 \%$ & $2.3779 \%$ & $2.7712 \%$ & $2.2554 \%$ & $2.4234 \%$ \\
\hline 8 & $2.0049 \%$ & $1.3604 \%$ & $1.7049 \%$ & $2.5184 \%$ & $2.5739 \%$ & $1.8145 \%$ & $1.4555 \%$ & $2.4577 \%$ & $2.4630 \%$ \\
\hline 9 & $3.8778 \%$ & $2.8677 \%$ & $3.9251 \%$ & $2.7354 \%$ & $3.3234 \%$ & $3.0317 \%$ & $3.8240 \%$ & $2.6306 \%$ & $1.5576 \%$ \\
\hline 10 & $3.7175 \%$ & $3.9114 \%$ & $3.8002 \%$ & $3.7166 \%$ & $3.6549 \%$ & $3.8491 \%$ & $3.8080 \%$ & $3.6201 \%$ & $2.8378 \%$ \\
\hline 11 & $3.6551 \%$ & $4.2086 \%$ & $4.1330 \%$ & $3.9667 \%$ & $4.3183 \%$ & $3.7345 \%$ & $3.7956 \%$ & $4.9456 \%$ & $2.9560 \%$ \\
\hline 12 & $4.0901 \%$ & $4.0228 \%$ & $3.9856 \%$ & $3.8900 \%$ & $4.2449 \%$ & $4.0920 \%$ & $5.9880 \%$ & $5.8679 \%$ & $9.1381 \%$ \\
\hline 13 & $5.7873 \%$ & $5.6201 \%$ & $5.8026 \%$ & $5.4926 \%$ & $5.8260 \%$ & $5.8038 \%$ & $5.5565 \%$ & $5.4150 \%$ & $11.2226 \%$ \\
\hline 14 & $6.1176 \%$ & $6.1318 \%$ & $6.1322 \%$ & $6.3200 \%$ & $6.6593 \%$ & $6.1142 \%$ & $6.1216 \%$ & $6.3371 \%$ & $3.8839 \%$ \\
\hline 15 & $5.7822 \%$ & $6.0222 \%$ & $5.6167 \%$ & $6.8351 \%$ & $5.2472 \%$ & $6.5790 \%$ & $6.7016 \%$ & $6.2981 \%$ & $7.4485 \%$ \\
\hline 16 & $6.2943 \%$ & $6.9993 \%$ & $6.9480 \%$ & $6.9451 \%$ & $6.8002 \%$ & $7.3661 \%$ & $7.1026 \%$ & $6.4792 \%$ & $4.3722 \%$ \\
\hline 17 & $6.2186 \%$ & $7.3597 \%$ & $7.2062 \%$ & $7.1843 \%$ & $7.4945 \%$ & $7.5009 \%$ & $7.1847 \%$ & $7.0262 \%$ & $10.9582 \%$ \\
\hline 18 & $6.7038 \%$ & $7.3196 \%$ & $7.2442 \%$ & $6.7083 \%$ & $6.4414 \%$ & $6.4569 \%$ & $7.4109 \%$ & $7.0492 \%$ & $8.5249 \%$ \\
\hline Average & $3.8004 \%$ & $3.9434 \%$ & $4.0285 \%$ & $3.9314 \%$ & $3.8313 \%$ & $3.9405 \%$ & $4.0237 \%$ & $3.9485 \%$ & $4.6322 \%$ \\
\hline
\end{tabular}

Table 5 This table shows the accuracy of monthly forecasts of US tourist arrivals from Japan. The Absolute Percentage Error (APE) is calculated at forecasting horizon 1 to 18 months (1.5 years). The ARFIMA, ARIMA, and SARIMA models are all estimated by the data from Jan 1996 to Jun 2013 (210 months).

\begin{tabular}{rrrr}
\hline horizon & ARFIMA & \multicolumn{1}{c}{ ARIMA } & SARIMA \\
\hline 1 & $0.4865 \%$ & $18.5198 \%$ & $3.0922 \%$ \\
2 & $2.0643 \%$ & $9.3111 \%$ & $2.1239 \%$ \\
3 & $2.5707 \%$ & $14.2966 \%$ & $2.7580 \%$ \\
4 & $3.5864 \%$ & $18.5518 \%$ & $3.9179 \%$ \\
5 & $6.8951 \%$ & $14.5906 \%$ & $4.2308 \%$ \\
6 & $9.4675 \%$ & $8.5771 \%$ & $3.6135 \%$ \\
7 & $10.2902 \%$ & $0.5218 \%$ & $3.0747 \%$ \\
8 & $10.9009 \%$ & $7.8582 \%$ & $3.3738 \%$ \\
9 & $13.0405 \%$ & $6.9061 \%$ & $4.3382 \%$ \\
10 & $13.7182 \%$ & $3.3191 \%$ & $4.5412 \%$ \\
11 & $16.9740 \%$ & $1.8422 \%$ & $4.9572 \%$ \\
12 & $17.7309 \%$ & $1.2963 \%$ & $5.8497 \%$ \\
13 & $18.0973 \%$ & $2.6266 \%$ & $7.0228 \%$ \\
14 & $18.5660 \%$ & $1.5557 \%$ & $6.7218 \%$ \\
15 & $19.6086 \%$ & $13.2230 \%$ & $8.1238 \%$ \\
16 & $22.1751 \%$ & $8.0824 \%$ & $8.1422 \%$
\end{tabular}




\begin{tabular}{rrrr}
17 & $26.4561 \%$ & $11.7477 \%$ & $9.6670 \%$ \\
18 & $31.0637 \%$ & $19.9274 \%$ & $10.4409 \%$ \\
\hline Average & $13.5384 \%$ & $9.0419 \%$ & $5.3328 \%$ \\
\hline
\end{tabular}

Table 6 This table shows the accuracy of monthly forecasts of US tourist arrivals from P.R.China. The Absolute Percentage Error (APE) is calculated at forecasting horizon 1 to 18 months (1.5 years). The $p$ NN-HP, $p$ NN-WT and $p$ NN-MA models are all estimated by the data from Jan 1996 to Jun 2013 (210 months).

\begin{tabular}{|c|c|c|c|c|c|c|c|c|c|}
\hline & \multicolumn{4}{|c|}{$p$ NN HP } & \multicolumn{4}{|c|}{$p$ NN WT } & \multirow{2}{*}{$p$ NN MA } \\
\hline horizon & 100 & 1600 & 3600 & 129600 & 3 & 4 & 5 & 6 & \\
\hline 1 & $1.4738 \%$ & $2.2359 \%$ & $2.0059 \%$ & $2.8848 \%$ & $0.9956 \%$ & $0.0812 \%$ & $0.6563 \%$ & $0.9732 \%$ & $2.7280 \%$ \\
\hline 2 & $2.4523 \%$ & $2.8736 \%$ & $2.3594 \%$ & $5.4266 \%$ & $0.5300 \%$ & $3.3011 \%$ & $1.2894 \%$ & $3.6583 \%$ & $5.6464 \%$ \\
\hline 3 & $4.6712 \%$ & $2.2179 \%$ & $2.7901 \%$ & $2.9574 \%$ & $1.3336 \%$ & $2.2560 \%$ & $1.7138 \%$ & $1.4117 \%$ & $11.0425 \%$ \\
\hline 4 & $2.2918 \%$ & $2.1086 \%$ & $1.3180 \%$ & $2.8814 \%$ & $1.3688 \%$ & $1.0805 \%$ & $1.1245 \%$ & $1.7318 \%$ & $7.6005 \%$ \\
\hline 5 & $2.1485 \%$ & $2.9594 \%$ & $1.1641 \%$ & $2.5909 \%$ & $1.3180 \%$ & $1.2627 \%$ & $1.2153 \%$ & $1.8928 \%$ & $7.5157 \%$ \\
\hline 6 & $0.6943 \%$ & $2.1607 \%$ & $2.2778 \%$ & $2.8697 \%$ & $0.5464 \%$ & $1.5500 \%$ & $1.9015 \%$ & $1.6624 \%$ & $1.5161 \%$ \\
\hline 7 & $0.6677 \%$ & $2.0638 \%$ & $2.1727 \%$ & $2.8963 \%$ & $0.0542 \%$ & $1.8700 \%$ & $1.5801 \%$ & $1.3611 \%$ & $0.7167 \%$ \\
\hline 8 & $2.0986 \%$ & $2.2919 \%$ & $2.2944 \%$ & $1.6247 \%$ & $1.5961 \%$ & $1.8676 \%$ & $1.1848 \%$ & $1.3858 \%$ & $6.4989 \%$ \\
\hline 9 & $0.0362 \%$ & $2.2517 \%$ & $2.3559 \%$ & $1.1620 \%$ & $2.1071 \%$ & $1.6034 \%$ & $1.7449 \%$ & $1.9099 \%$ & $6.4290 \%$ \\
\hline 10 & $1.2598 \%$ & $2.5191 \%$ & $2.7276 \%$ & $1.5526 \%$ & $2.2008 \%$ & $2.9325 \%$ & $2.8823 \%$ & $2.9619 \%$ & $9.3558 \%$ \\
\hline 11 & $2.8297 \%$ & $5.7708 \%$ & $6.4601 \%$ & $1.2873 \%$ & $3.7010 \%$ & $4.7387 \%$ & $3.2893 \%$ & $4.1472 \%$ & $7.3130 \%$ \\
\hline 12 & $1.7281 \%$ & $1.8603 \%$ & $1.7315 \%$ & $0.7695 \%$ & $3.5486 \%$ & $4.7882 \%$ & $3.3827 \%$ & $3.1286 \%$ & $12.7059 \%$ \\
\hline 13 & $3.4168 \%$ & $3.4886 \%$ & $3.4277 \%$ & $2.8247 \%$ & $2.9467 \%$ & $4.8015 \%$ & $4.4521 \%$ & $3.2130 \%$ & $11.1119 \%$ \\
\hline 14 & $2.2418 \%$ & $1.6473 \%$ & $1.4741 \%$ & $3.7678 \%$ & $5.8597 \%$ & $3.5690 \%$ & $3.2767 \%$ & $3.0562 \%$ & $11.0950 \%$ \\
\hline 15 & $3.7302 \%$ & $2.6282 \%$ & $2.3762 \%$ & $2.4691 \%$ & $4.2891 \%$ & $5.8927 \%$ & $5.0202 \%$ & $5.0731 \%$ & $13.4263 \%$ \\
\hline 16 & $1.0693 \%$ & $0.0094 \%$ & $2.1627 \%$ & $2.0201 \%$ & $3.7017 \%$ & $3.1070 \%$ & $6.1266 \%$ & $4.8330 \%$ & $14.7797 \%$ \\
\hline 17 & $2.6976 \%$ & $2.1397 \%$ & $2.0130 \%$ & $2.6595 \%$ & $4.6959 \%$ & $4.5980 \%$ & $5.2372 \%$ & $5.3744 \%$ & $12.2693 \%$ \\
\hline 18 & $2.6046 \%$ & $2.2771 \%$ & $2.1770 \%$ & $3.7490 \%$ & $3.2764 \%$ & $4.8806 \%$ & $5.5901 \%$ & $5.6262 \%$ & $5.4539 \%$ \\
\hline Average & $2.1173 \%$ & $2.4169 \%$ & $2.4049 \%$ & $2.5774 \%$ & $2.4483 \%$ & $3.0100 \%$ & $2.8704 \%$ & $2.9667 \%$ & $8.1780 \%$ \\
\hline
\end{tabular}

Table 7 This table shows the accuracy of monthly forecasts of US tourist arrivals from P.R.China. The Absolute Percentage Error (APE) is calculated at forecasting horizon 1 to 18 months (1.5 years). The ARFIMA, ARIMA, and SARIMA models are all estimated by the data from Jan 1996 to Jun 2013 (210 months).

\begin{tabular}{rrrr}
\hline horizon & ARFIMA & \multicolumn{1}{c}{ ARIMA } & SARIMA \\
\hline 1 & $2.8896 \%$ & $1.3820 \%$ & $1.6642 \%$ \\
2 & $3.8579 \%$ & $1.0634 \%$ & $2.9507 \%$ \\
3 & $6.8948 \%$ & $1.3463 \%$ & $3.5123 \%$ \\
4 & $7.4817 \%$ & $2.7155 \%$ & $2.8821 \%$ \\
5 & $9.0310 \%$ & $3.9356 \%$ & $3.1849 \%$ \\
6 & $13.9876 \%$ & $4.7293 \%$ & $3.0814 \%$ \\
7 & $14.5515 \%$ & $4.9104 \%$ & $2.9859 \%$ \\
8 & $18.5565 \%$ & $5.5026 \%$ & $4.0820 \%$ \\
9 & $18.9052 \%$ & $8.2006 \%$ & $4.2460 \%$ \\
10 & $19.6476 \%$ & $12.7071 \%$ & $5.5225 \%$
\end{tabular}




\begin{tabular}{rrrr}
11 & $22.8445 \%$ & $13.2741 \%$ & $6.8778 \%$ \\
12 & $28.5510 \%$ & $14.3330 \%$ & $6.9570 \%$ \\
13 & $29.9216 \%$ & $14.5949 \%$ & $7.6545 \%$ \\
14 & $30.3773 \%$ & $16.4351 \%$ & $7.5273 \%$ \\
15 & $34.7792 \%$ & $16.5667 \%$ & $8.7501 \%$ \\
16 & $35.5623 \%$ & $23.8547 \%$ & $8.8388 \%$ \\
17 & $39.8836 \%$ & $27.2654 \%$ & $9.8940 \%$ \\
18 & $43.0803 \%$ & $34.1910 \%$ & $10.2642 \%$ \\
\hline Average & $21.1557 \%$ & $11.5004 \%$ & $5.6042 \%$ \\
\hline
\end{tabular}

Table 8 This table shows the accuracy of monthly forecasts of US tourist arrivals from Canada. The Absolute Percentage Error (APE) is calculated at forecasting horizon 1 to 18 months (1.5 years). The $p \mathrm{NN}-\mathrm{HP}, p \mathrm{NN}-\mathrm{WT}$ and $p \mathrm{NN}-\mathrm{MA}$ models are all estimated by the data from Jan 1996 to Jun 2013 (210 months).

\begin{tabular}{|c|c|c|c|c|c|c|c|c|c|}
\hline \multirow[b]{2}{*}{ horizon } & \multicolumn{4}{|c|}{$p$ NN HP } & \multicolumn{4}{|c|}{$p$ NN WT } & \multirow{2}{*}{$p \mathrm{NN}$ MA } \\
\hline & 100 & 1600 & 3600 & 129600 & 3 & 4 & 5 & 6 & \\
\hline 1 & $0.5876 \%$ & $0.7549 \%$ & $0.8528 \%$ & $1.8342 \%$ & $1.3697 \%$ & $1.4540 \%$ & $0.3280 \%$ & $0.4781 \%$ & $5.0198 \%$ \\
\hline 2 & $1.6982 \%$ & $2.0527 \%$ & $2.0492 \%$ & $0.3813 \%$ & $1.0996 \%$ & $2.7130 \%$ & $1.3349 \%$ & $0.5141 \%$ & $4.9723 \%$ \\
\hline 3 & $0.8989 \%$ & $1.1231 \%$ & $2.0282 \%$ & $1.2038 \%$ & $1.4774 \%$ & $1.8661 \%$ & $1.3855 \%$ & $1.2028 \%$ & $0.0582 \%$ \\
\hline 4 & $1.4483 \%$ & $2.2848 \%$ & $1.6537 \%$ & $1.6576 \%$ & $1.8615 \%$ & $1.4458 \%$ & $1.7282 \%$ & $1.6035 \%$ & $2.4557 \%$ \\
\hline 5 & $1.2068 \%$ & $2.6179 \%$ & $2.4912 \%$ & $2.1193 \%$ & $1.5662 \%$ & $1.9774 \%$ & $1.7214 \%$ & $2.1586 \%$ & $3.5488 \%$ \\
\hline 6 & $0.9329 \%$ & $2.7901 \%$ & $2.5614 \%$ & $1.6593 \%$ & $2.0992 \%$ & $1.8354 \%$ & $2.0999 \%$ & $1.7086 \%$ & $5.9304 \%$ \\
\hline 7 & $0.6109 \%$ & $1.4625 \%$ & $1.2730 \%$ & $1.2696 \%$ & $2.3063 \%$ & $2.5997 \%$ & $2.2925 \%$ & $1.2223 \%$ & $2.2629 \%$ \\
\hline 8 & $1.2003 \%$ & $2.2605 \%$ & $1.9048 \%$ & $1.6215 \%$ & $2.5261 \%$ & $2.8441 \%$ & $2.3703 \%$ & $1.6814 \%$ & $0.9145 \%$ \\
\hline 9 & $0.2071 \%$ & $2.9506 \%$ & $2.7348 \%$ & $3.1387 \%$ & $2.9473 \%$ & $3.7996 \%$ & $2.3013 \%$ & $3.2054 \%$ & $2.2038 \%$ \\
\hline 10 & $0.1927 \%$ & $3.8076 \%$ & $3.4699 \%$ & $4.1844 \%$ & $2.7320 \%$ & $3.6803 \%$ & $3.0351 \%$ & $4.2295 \%$ & $3.9121 \%$ \\
\hline 11 & $0.2540 \%$ & $3.9938 \%$ & $3.8046 \%$ & $4.0029 \%$ & $3.6149 \%$ & $3.8840 \%$ & $3.2876 \%$ & $3.5351 \%$ & $4.2601 \%$ \\
\hline 12 & $0.2818 \%$ & $2.6604 \%$ & $2.7816 \%$ & $3.1039 \%$ & $3.6329 \%$ & $4.1907 \%$ & $4.7214 \%$ & $3.4208 \%$ & $9.7813 \%$ \\
\hline 13 & $1.5186 \%$ & $4.7459 \%$ & $4.3479 \%$ & $6.0063 \%$ & $4.7439 \%$ & $4.9335 \%$ & $5.8298 \%$ & $6.1526 \%$ & $9.6440 \%$ \\
\hline 14 & $2.5604 \%$ & $6.7764 \%$ & $6.1757 \%$ & $6.1328 \%$ & $4.1963 \%$ & $5.6953 \%$ & $5.4062 \%$ & $5.1058 \%$ & $9.4637 \%$ \\
\hline 15 & $2.9147 \%$ & $6.7987 \%$ & $6.1364 \%$ & $5.4831 \%$ & $5.2767 \%$ & $5.7651 \%$ & $5.6777 \%$ & $5.6479 \%$ & $8.3466 \%$ \\
\hline 16 & $3.4394 \%$ & $5.7385 \%$ & $5.4400 \%$ & $6.6282 \%$ & $5.6758 \%$ & $5.2407 \%$ & $5.3021 \%$ & $6.7161 \%$ & $8.0811 \%$ \\
\hline 17 & $3.4177 \%$ & $6.2754 \%$ & $5.8506 \%$ & $6.4451 \%$ & $5.8757 \%$ & $6.2370 \%$ & $6.5331 \%$ & $6.6031 \%$ & $8.3315 \%$ \\
\hline 18 & $3.6146 \%$ & $6.2959 \%$ & $6.0332 \%$ & $7.3911 \%$ & $5.8067 \%$ & $6.4584 \%$ & $6.2678 \%$ & $6.8558 \%$ & $10.9011 \%$ \\
\hline Average & $1.4992 \%$ & $3.6328 \%$ & $3.4216 \%$ & $3.5702 \%$ & $3.2671 \%$ & $3.7011 \%$ & $3.4235 \%$ & $3.4467 \%$ & $5.5604 \%$ \\
\hline
\end{tabular}

Table 9 This table shows the accuracy of monthly forecasts of US tourist arrivals from Canada. The Absolute Percentage Error (APE) is calculated at forecasting horizon 1 to 18 months (1.5 years). The ARFIMA, ARIMA, and SARIMA models are all estimated by the data from Jan 1996 to Jun 2013 (210 months).

\begin{tabular}{rrrr}
\hline horizon & ARFIMA & ARIMA & SARIMA \\
\hline 1 & $0.2743 \%$ & $0.3820 \%$ & $1.2123 \%$ \\
2 & $0.7676 \%$ & $1.0634 \%$ & $1.6951 \%$ \\
3 & $1.9123 \%$ & $1.3463 \%$ & $1.3184 \%$ \\
4 & $2.4996 \%$ & $2.7155 \%$ & $1.9413 \%$ \\
5 & $5.2153 \%$ & $3.9356 \%$ & $2.5962 \%$ \\
6 & $5.2406 \%$ & $4.7293 \%$ & $2.8716 \%$
\end{tabular}




\begin{tabular}{rrrr}
7 & $6.4020 \%$ & $4.9104 \%$ & $2.4193 \%$ \\
8 & $8.6295 \%$ & $5.5026 \%$ & $2.8596 \%$ \\
9 & $9.3311 \%$ & $8.2006 \%$ & $3.7291 \%$ \\
10 & $9.9407 \%$ & $12.7071 \%$ & $4.7174 \%$ \\
11 & $11.1286 \%$ & $13.2741 \%$ & $5.0036 \%$ \\
12 & $11.3902 \%$ & $14.3330 \%$ & $5.4816 \%$ \\
13 & $12.1584 \%$ & $14.5949 \%$ & $6.7887 \%$ \\
14 & $14.1502 \%$ & $16.4351 \%$ & $7.4634 \%$ \\
15 & $15.0727 \%$ & $16.5667 \%$ & $7.6078 \%$ \\
16 & $19.3988 \%$ & $23.8547 \%$ & $8.6832 \%$ \\
17 & $24.6458 \%$ & $27.2654 \%$ & $9.7710 \%$ \\
18 & $31.7562 \%$ & $34.1910 \%$ & $11.4156 \%$ \\
\hline Average & $10.5508 \%$ & $11.4449 \%$ & $4.8653 \%$ \\
\hline
\end{tabular}

Table 10 This table shows the accuracy of monthly forecasts of US tourist arrivals from Mexico. The Absolute Percentage Error (APE) is calculated at forecasting horizon 1 to 18 months (1.5 years). The $p$ NN-HP, $p$ NN-WT and $p$ NN-MA models are all estimated by the data from Jan 1996 to Jun 2013 (210 months).

\begin{tabular}{|c|c|c|c|c|c|c|c|c|c|}
\hline \multirow[b]{2}{*}{ horizon } & \multicolumn{4}{|c|}{$p$ NN HP } & \multicolumn{4}{|c|}{$p$ NN WT } & \multirow{2}{*}{$p$ NN MA } \\
\hline & $\lambda=100$ & $\lambda=1600$ & $\lambda=3600$ & $\lambda=129600$ & level 3 & level 4 & level 5 & level 6 & \\
\hline 1 & $1.0303 \%$ & $0.7549 \%$ & $0.8528 \%$ & $1.8183 \%$ & $1.2233 \%$ & $1.0932 \%$ & $0.4273 \%$ & $0.5396 \%$ & $1.6852 \%$ \\
\hline 2 & $1.3432 \%$ & $2.0527 \%$ & $2.0492 \%$ & $2.4463 \%$ & $1.8404 \%$ & $2.1276 \%$ & $1.4575 \%$ & $1.4531 \%$ & $4.0865 \%$ \\
\hline 3 & $1.4270 \%$ & $1.1231 \%$ & $2.0282 \%$ & $0.6498 \%$ & $1.0411 \%$ & $2.1061 \%$ & $1.0590 \%$ & $1.3539 \%$ & $2.0481 \%$ \\
\hline 4 & $1.6341 \%$ & $2.2848 \%$ & $1.6537 \%$ & $2.6260 \%$ & $1.6634 \%$ & $2.7180 \%$ & $2.7834 \%$ & $2.1080 \%$ & $5.1358 \%$ \\
\hline 5 & $1.8037 \%$ & $2.6179 \%$ & $2.4912 \%$ & $3.8036 \%$ & $1.6326 \%$ & $3.7678 \%$ & $2.3031 \%$ & $2.6332 \%$ & $4.3871 \%$ \\
\hline 6 & $2.0126 \%$ & $2.7901 \%$ & $2.5614 \%$ & $4.3407 \%$ & $1.0443 \%$ & $3.4638 \%$ & $2.8478 \%$ & $2.5143 \%$ & $1.5918 \%$ \\
\hline 7 & $1.1602 \%$ & $1.4625 \%$ & $1.2730 \%$ & $3.1781 \%$ & $1.3287 \%$ & $3.4083 \%$ & $2.3047 \%$ & $2.8762 \%$ & $0.5056 \%$ \\
\hline 8 & $2.4166 \%$ & $2.2605 \%$ & $1.9048 \%$ & $3.4777 \%$ & $1.2940 \%$ & $3.8321 \%$ & $2.9106 \%$ & $2.2913 \%$ & $0.3607 \%$ \\
\hline 9 & $1.6581 \%$ & $2.9506 \%$ & $2.7348 \%$ & $4.2646 \%$ & $1.4287 \%$ & $4.5479 \%$ & $3.4219 \%$ & $3.8964 \%$ & $2.3973 \%$ \\
\hline 10 & $2.7440 \%$ & $3.8076 \%$ & $3.4699 \%$ & $4.8321 \%$ & $1.8148 \%$ & $5.3376 \%$ & $4.3598 \%$ & $4.9320 \%$ & $0.2436 \%$ \\
\hline 11 & $3.2269 \%$ & $3.9938 \%$ & $3.8046 \%$ & $5.2973 \%$ & $1.7748 \%$ & $5.2688 \%$ & $4.4136 \%$ & $4.7927 \%$ & $2.6506 \%$ \\
\hline 12 & $2.4804 \%$ & $2.6604 \%$ & $2.7816 \%$ & $4.8132 \%$ & $1.8505 \%$ & $5.3872 \%$ & $4.2595 \%$ & $4.3876 \%$ & $4.8089 \%$ \\
\hline 13 & $4.5861 \%$ & $4.7459 \%$ & $4.3479 \%$ & $5.5715 \%$ & $2.8987 \%$ & $5.9117 \%$ & $5.4460 \%$ & $4.2619 \%$ & $3.2915 \%$ \\
\hline 14 & $5.8705 \%$ & $6.7764 \%$ & $6.1757 \%$ & $8.6470 \%$ & $4.0512 \%$ & $5.2926 \%$ & $5.9064 \%$ & $5.7734 \%$ & $7.3471 \%$ \\
\hline 15 & $5.6514 \%$ & $6.7987 \%$ & $6.1364 \%$ & $7.4945 \%$ & $4.6904 \%$ & $6.5939 \%$ & $5.3693 \%$ & $5.3088 \%$ & $5.8922 \%$ \\
\hline 16 & $5.4679 \%$ & $5.7385 \%$ & $5.4400 \%$ & $7.3922 \%$ & $5.6796 \%$ & $6.4421 \%$ & $5.1079 \%$ & $5.6051 \%$ & $5.3964 \%$ \\
\hline 17 & $5.1059 \%$ & $6.2754 \%$ & $5.8506 \%$ & $9.0279 \%$ & $5.7356 \%$ & $7.0455 \%$ & $7.2478 \%$ & $7.0145 \%$ & $13.2501 \%$ \\
\hline 18 & $6.6773 \%$ & $6.2959 \%$ & $6.0332 \%$ & $8.5478 \%$ & $7.1605 \%$ & $7.3730 \%$ & $7.7003 \%$ & $7.0234 \%$ & $6.9257 \%$ \\
\hline Average & $3.1276 \%$ & $3.6328 \%$ & $3.4216 \%$ & $4.8664 \%$ & $2.4856 \%$ & $4.5782 \%$ & $3.8249 \%$ & $3.8252 \%$ & $4.0002 \%$ \\
\hline
\end{tabular}

Table 11 This table shows the accuracy of monthly forecasts of US tourist arrivals from Mexico. The Absolute Percentage Error (APE) is calculated at forecasting horizon 1 to 18 months (1.5 years). The ARFIMA, ARIMA, and SARIMA models are all estimated by the data from Jan 1996 to Jun 2013 (210 months).

horizon ARFIMA ARIMA SARIMA 


\begin{tabular}{rrrr}
1 & $1.5598 \%$ & $9.4306 \%$ & $1.8559 \%$ \\
2 & $3.3231 \%$ & $6.2667 \%$ & $2.5860 \%$ \\
3 & $5.9260 \%$ & $8.8243 \%$ & $2.5079 \%$ \\
4 & $14.2209 \%$ & $13.9717 \%$ & $4.6182 \%$ \\
5 & $14.6464 \%$ & $16.0893 \%$ & $5.1069 \%$ \\
6 & $15.1554 \%$ & $10.7261 \%$ & $4.4589 \%$ \\
7 & $16.0494 \%$ & $19.3084 \%$ & $4.8050 \%$ \\
8 & $16.6518 \%$ & $14.9878 \%$ & $4.7625 \%$ \\
9 & $17.9664 \%$ & $24.2640 \%$ & $6.3210 \%$ \\
10 & $18.6219 \%$ & $20.0341 \%$ & $6.3816 \%$ \\
11 & $19.4014 \%$ & $23.0710 \%$ & $7.0632 \%$ \\
12 & $21.9862 \%$ & $34.7013 \%$ & $8.1924 \%$ \\
13 & $23.0783 \%$ & $36.2280 \%$ & $9.1243 \%$ \\
14 & $23.6760 \%$ & $31.1101 \%$ & $10.0570 \%$ \\
15 & $27.8694 \%$ & $26.6214 \%$ & $9.8569 \%$ \\
16 & $29.6739 \%$ & $27.5956 \%$ & $9.9581 \%$ \\
17 & $30.4218 \%$ & $30.8445 \%$ & $11.6200 \%$ \\
18 & $37.8498 \%$ & $38.5734 \%$ & $12.7418 \%$ \\
\hline Average & $18.7821 \%$ & $21.8138 \%$ & $6.7598 \%$ \\
\hline & & & \\
\hline
\end{tabular}

Table 12 This table shows the accuracy of monthly forecasts of US tourist arrivals from France. The Absolute Percentage Error (APE) is calculated at forecasting horizon 1 to 18 months (1.5 years). The $p$ NN-HP, $p$ NN-WT and $p$ NN-MA models are all estimated by the data from Jan 1996 to Jun 2013 (210 months)

\begin{tabular}{|c|c|c|c|c|c|c|c|c|c|}
\hline \multirow[b]{2}{*}{ horizon } & \multicolumn{4}{|c|}{$p$ NN HP } & \multicolumn{4}{|c|}{$p$ NN WT } & \multirow{2}{*}{$p$ NN MA } \\
\hline & 100 & 1600 & 3600 & 129600 & 3 & 4 & 5 & 6 & \\
\hline 1 & $3.2027 \%$ & $4.1723 \%$ & $3.4533 \%$ & $5.2630 \%$ & $0.9478 \%$ & $6.0442 \%$ & $4.5824 \%$ & $4.6654 \%$ & $12.6512 \%$ \\
\hline 2 & $1.5821 \%$ & $2.4158 \%$ & $0.6651 \%$ & $2.3053 \%$ & $1.3434 \%$ & $3.3115 \%$ & $2.2900 \%$ & $2.1426 \%$ & $0.4667 \%$ \\
\hline 3 & $0.7468 \%$ & $1.1671 \%$ & $0.6886 \%$ & $2.2894 \%$ & $1.7170 \%$ & $2.3978 \%$ & $2.4377 \%$ & $2.1139 \%$ & $2.5901 \%$ \\
\hline 4 & $1.2826 \%$ & $0.4886 \%$ & $1.0397 \%$ & $1.1068 \%$ & $0.9280 \%$ & $1.3175 \%$ & $0.8926 \%$ & $2.7692 \%$ & $1.2201 \%$ \\
\hline 5 & $2.5993 \%$ & $2.1395 \%$ & $2.1628 \%$ & $2.0750 \%$ & $2.0602 \%$ & $2.9168 \%$ & $1.9423 \%$ & $2.2033 \%$ & $8.4099 \%$ \\
\hline 6 & $2.6886 \%$ & $2.4382 \%$ & $2.3765 \%$ & $2.2685 \%$ & $0.4719 \%$ & $3.0303 \%$ & $2.2940 \%$ & $2.8161 \%$ & $5.7287 \%$ \\
\hline 7 & $4.2706 \%$ & $4.1083 \%$ & $4.0246 \%$ & $3.4881 \%$ & $2.2615 \%$ & $4.2408 \%$ & $3.9043 \%$ & $2.9176 \%$ & $3.9227 \%$ \\
\hline 8 & $2.3922 \%$ & $4.1707 \%$ & $4.0328 \%$ & $3.8053 \%$ & $3.9929 \%$ & $3.4214 \%$ & $3.6045 \%$ & $3.1091 \%$ & $2.7618 \%$ \\
\hline 9 & $3.9190 \%$ & $2.9572 \%$ & $2.8016 \%$ & $3.4686 \%$ & $3.9666 \%$ & $4.0980 \%$ & $3.7945 \%$ & $3.0317 \%$ & $8.8035 \%$ \\
\hline 10 & $3.8011 \%$ & $4.0665 \%$ & $3.8909 \%$ & $3.3178 \%$ & $4.4975 \%$ & $4.8297 \%$ & $4.0877 \%$ & $3.4178 \%$ & $2.6140 \%$ \\
\hline 11 & $2.7181 \%$ & $2.6640 \%$ & $2.3041 \%$ & $2.0697 \%$ & $4.2153 \%$ & $6.1107 \%$ & $2.2561 \%$ & $3.9401 \%$ & $8.2364 \%$ \\
\hline 12 & $3.2637 \%$ & $2.8801 \%$ & $2.6060 \%$ & $2.0367 \%$ & $3.8353 \%$ & $5.7709 \%$ & $3.8882 \%$ & $4.5028 \%$ & $11.5365 \%$ \\
\hline 13 & $7.9898 \%$ & $8.6338 \%$ & $9.2439 \%$ & $7.5091 \%$ & $5.9379 \%$ & $10.8231 \%$ & $8.4801 \%$ & $8.6239 \%$ & $12.5583 \%$ \\
\hline 14 & $7.0039 \%$ & $8.4351 \%$ & $4.7674 \%$ & $2.8099 \%$ & $6.6998 \%$ & $8.9735 \%$ & $7.5532 \%$ & $7.3956 \%$ & $3.7810 \%$ \\
\hline 15 & $5.8007 \%$ & $6.9195 \%$ & $5.4257 \%$ & $4.8649 \%$ & $5.8841 \%$ & $6.0714 \%$ & $5.6052 \%$ & $4.2967 \%$ & $3.5145 \%$ \\
\hline 16 & $5.4728 \%$ & $5.4108 \%$ & $5.3075 \%$ & $4.4898 \%$ & $4.6002 \%$ & $5.4515 \%$ & $5.4583 \%$ & $4.4602 \%$ & $7.4224 \%$ \\
\hline 17 & $6.5138 \%$ & $6.6733 \%$ & $5.9333 \%$ & $5.6390 \%$ & $4.8447 \%$ & $6.6754 \%$ & $6.4543 \%$ & $5.5396 \%$ & $12.4412 \%$ \\
\hline 18 & $7.2474 \%$ & $6.1522 \%$ & $6.7785 \%$ & $5.2820 \%$ & $3.6605 \%$ & $7.6003 \%$ & $7.3551 \%$ & $6.1803 \%$ & $11.6435 \%$ \\
\hline Average & $4.0275 \%$ & $4.2163 \%$ & $3.7501 \%$ & $3.5605 \%$ & $3.4369 \%$ & $5.1714 \%$ & $4.2711 \%$ & $4.1181 \%$ & $6.6835 \%$ \\
\hline
\end{tabular}


Table 13 This table shows the accuracy of monthly forecasts of US tourist arrivals from France. The Absolute Percentage Error (APE) is calculated at forecasting horizon 1 to 18 months (1.5 years). The ARFIMA, ARIMA, and SARIMA models are all estimated by the data from Jan 1996 to Jun 2013 (210 months).

\begin{tabular}{rrrr}
\hline horizon & ARFIMA & \multicolumn{1}{c}{ ARIMA } & SARIMA \\
\hline 1 & $0.0563 \%$ & $0.2287 \%$ & $4.1152 \%$ \\
2 & $4.3767 \%$ & $3.0465 \%$ & $2.1769 \%$ \\
3 & $3.8329 \%$ & $3.1798 \%$ & $2.1056 \%$ \\
4 & $3.2855 \%$ & $4.3094 \%$ & $1.6945 \%$ \\
5 & $7.9836 \%$ & $7.8926 \%$ & $3.8532 \%$ \\
6 & $9.4953 \%$ & $8.1076 \%$ & $3.7923 \%$ \\
7 & $5.7207 \%$ & $10.1122 \%$ & $4.4519 \%$ \\
8 & $14.1408 \%$ & $16.0817 \%$ & $5.5921 \%$ \\
9 & $17.0210 \%$ & $17.5074 \%$ & $6.4881 \%$ \\
10 & $16.4819 \%$ & $18.4163 \%$ & $6.3110 \%$ \\
11 & $24.3493 \%$ & $19.2968 \%$ & $7.1055 \%$ \\
12 & $22.8291 \%$ & $20.0931 \%$ & $7.5675 \%$ \\
13 & $22.7435 \%$ & $22.7652 \%$ & $11.3917 \%$ \\
14 & $27.0123 \%$ & $27.0609 \%$ & $10.1357 \%$ \\
15 & $28.3679 \%$ & $27.4863 \%$ & $9.4761 \%$ \\
16 & $30.4069 \%$ & $29.7150 \%$ & $9.8359 \%$ \\
17 & $65.1216 \%$ & $68.4182 \%$ & $17.6595 \%$ \\
18 & $54.7041 \%$ & $79.3608 \%$ & $17.8150 \%$ \\
\hline Average & $19.8850 \%$ & $21.2821 \%$ & $7.3093 \%$ \\
\hline & & &
\end{tabular}

Table 14 This table shows the accuracy of monthly forecasts of US tourist arrivals from UK. The Absolute Percentage Error (APE) is calculated at forecasting horizon 1 to 18 months (1.5 years). The $p \mathrm{NN}-\mathrm{HP}, p \mathrm{NN}-\mathrm{WT}$ and $p \mathrm{NN}-\mathrm{MA}$ models are all estimated by the data from Jan 1996 to Jun 2013 (210 months).

\begin{tabular}{rrrrr|rrrr|r}
\hline \multicolumn{7}{c}{$p$} & \multicolumn{4}{c|}{$p$ NN WT } & \multirow{2}{*}{$p$ NN MA } \\
\cline { 1 - 7 } horizon & 100 & 1600 & 3600 & 129600 & 3 & 4 & 5 & 6 & \\
\hline 1 & $0.9547 \%$ & $0.9703 \%$ & $1.2032 \%$ & $2.5245 \%$ & $3.1530 \%$ & $1.9045 \%$ & $0.5826 \%$ & $1.3244 \%$ & $5.9264 \%$ \\
2 & $2.2935 \%$ & $2.6239 \%$ & $0.1954 \%$ & $2.7551 \%$ & $2.9137 \%$ & $2.3669 \%$ & $0.3438 \%$ & $0.0441 \%$ & $6.1367 \%$ \\
3 & $1.4011 \%$ & $1.3260 \%$ & $1.2719 \%$ & $1.4380 \%$ & $2.3437 \%$ & $1.8266 \%$ & $1.0823 \%$ & $1.7709 \%$ & $3.8952 \%$ \\
4 & $2.0866 \%$ & $1.9839 \%$ & $1.9607 \%$ & $2.0994 \%$ & $1.8423 \%$ & $2.0276 \%$ & $2.0479 \%$ & $2.0634 \%$ & $0.7739 \%$ \\
5 & $2.3143 \%$ & $1.8793 \%$ & $1.9107 \%$ & $2.0739 \%$ & $2.2916 \%$ & $2.2884 \%$ & $1.8675 \%$ & $2.0137 \%$ & $5.9894 \%$ \\
6 & $2.1185 \%$ & $2.0289 \%$ & $1.9863 \%$ & $2.0821 \%$ & $2.4278 \%$ & $2.0422 \%$ & $2.9585 \%$ & $2.1163 \%$ & $1.9572 \%$ \\
7 & $2.8650 \%$ & $2.2785 \%$ & $3.0442 \%$ & $2.8512 \%$ & $3.3154 \%$ & $2.1144 \%$ & $3.0528 \%$ & $2.2176 \%$ & $3.4049 \%$ \\
8 & $1.9981 \%$ & $1.8580 \%$ & $2.1376 \%$ & $2.2103 \%$ & $3.0231 \%$ & $2.3600 \%$ & $3.1660 \%$ & $3.4752 \%$ & $6.6162 \%$ \\
9 & $2.7481 \%$ & $2.7443 \%$ & $3.8459 \%$ & $3.9382 \%$ & $3.0014 \%$ & $2.5530 \%$ & $3.6269 \%$ & $3.7656 \%$ & $5.1520 \%$ \\
10 & $4.4985 \%$ & $4.5747 \%$ & $4.4488 \%$ & $4.3688 \%$ & $4.4276 \%$ & $4.5775 \%$ & $4.5666 \%$ & $4.3854 \%$ & $5.4701 \%$ \\
11 & $3.2113 \%$ & $3.1344 \%$ & $4.5367 \%$ & $2.7828 \%$ & $4.8426 \%$ & $4.8023 \%$ & $4.4725 \%$ & $5.7785 \%$ & $2.8929 \%$ \\
12 & $3.6058 \%$ & $3.6662 \%$ & $3.6658 \%$ & $3.5160 \%$ & $3.1510 \%$ & $4.5092 \%$ & $3.5953 \%$ & $5.2182 \%$ & $9.6593 \%$ \\
13 & $8.4892 \%$ & $7.8067 \%$ & $8.4763 \%$ & $8.4566 \%$ & $4.4954 \%$ & $6.9303 \%$ & $7.8755 \%$ & $6.4571 \%$ & $9.0916 \%$ \\
14 & $7.4250 \%$ & $7.3572 \%$ & $7.3968 \%$ & $8.4128 \%$ & $8.4563 \%$ & $7.2718 \%$ & $7.8028 \%$ & $7.9026 \%$ & $12.6569 \%$ \\
15 & $5.9657 \%$ & $5.9083 \%$ & $5.8144 \%$ & $6.0798 \%$ & $5.1101 \%$ & $7.9106 \%$ & $7.9521 \%$ & $7.0552 \%$ & $6.6408 \%$
\end{tabular}




\begin{tabular}{rrrrr|rrrr|r}
16 & $6.5030 \%$ & $6.4474 \%$ & $6.4590 \%$ & $6.5717 \%$ & $5.8122 \%$ & $7.2904 \%$ & $7.3641 \%$ & $7.4789 \%$ & $4.0742 \%$ \\
17 & $6.7170 \%$ & $6.1681 \%$ & $6.2723 \%$ & $6.3987 \%$ & $6.6048 \%$ & $7.0164 \%$ & $7.3215 \%$ & $7.3525 \%$ & $11.3040 \%$ \\
18 & $6.8986 \%$ & $6.9548 \%$ & $6.9443 \%$ & $6.8136 \%$ & $6.6704 \%$ & $7.7903 \%$ & $7.8878 \%$ & $7.8924 \%$ & $6.1590 \%$ \\
\hline Average & $4.0052 \%$ & $3.8728 \%$ & $3.9761 \%$ & $4.1874 \%$ & $4.1046 \%$ & $4.3101 \%$ & $4.3092 \%$ & $4.3507 \%$ & $5.9889 \%$ \\
\hline
\end{tabular}

Table 15 This table shows the accuracy of monthly forecasts of US tourist arrivals from UK. The Absolute Percentage Error (APE) is calculated at forecasting horizon 1 to 18 months (1.5 years). The ARFIMA, ARIMA, and SARIMA models are all estimated by the data from Jan 1996 to Jun 2013 (210 months).

\begin{tabular}{rlll}
\hline horizon & ARFIMA & ARIMA & SARIMA \\
\hline 1 & $0.8388 \%$ & $3.0939 \%$ & $2.0433 \%$ \\
2 & $1.7065 \%$ & $0.7434 \%$ & $2.0112 \%$ \\
3 & $2.1569 \%$ & $0.5093 \%$ & $1.7293 \%$ \\
4 & $2.3598 \%$ & $3.2640 \%$ & $2.0463 \%$ \\
5 & $2.7858 \%$ & $2.2572 \%$ & $2.5156 \%$ \\
6 & $3.0822 \%$ & $4.0884 \%$ & $2.4444 \%$ \\
7 & $3.6274 \%$ & $6.3438 \%$ & $3.1923 \%$ \\
8 & $4.5189 \%$ & $15.6542 \%$ & $4.2743 \%$ \\
9 & $7.3903 \%$ & $10.0052 \%$ & $4.4337 \%$ \\
10 & $7.5344 \%$ & $4.9507 \%$ & $4.8912 \%$ \\
11 & $8.6988 \%$ & $7.2183 \%$ & $4.7610 \%$ \\
12 & $9.5324 \%$ & $5.9929 \%$ & $5.1011 \%$ \\
13 & $16.0177 \%$ & $19.1206 \%$ & $9.3834 \%$ \\
14 & $22.4998 \%$ & $27.2351 \%$ & $11.3106 \%$ \\
15 & $26.7107 \%$ & $23.2183 \%$ & $9.8515 \%$ \\
16 & $36.8453 \%$ & $41.1896 \%$ & $12.3669 \%$ \\
17 & $51.7933 \%$ & $74.1898 \%$ & $17.3762 \%$ \\
18 & $64.7718 \%$ & $69.1361 \%$ & $17.9927 \%$ \\
\hline Average & $15.1595 \%$ & $17.6784 \%$ & $6.5403 \%$ \\
\hline & & &
\end{tabular}

\section{$\underline{\text { Part } 2 \text { results }}$}

As discussed before, the second part evaluation is to show an average performance based on sliding window. As the experimental evaluation in Part 1 shows that the $p$ NN-HP model is slightly better than $p$ NN-WT and SARIMA outperforms the other two traditional models, in this part, we select $p \mathrm{NN}-\mathrm{HP}, p \mathrm{NN}-\mathrm{MA}$ and SARIMA models for the further evaluation. The Figure 11 shows the average MAPE and the RMSE error measures of the 12 tourist arrival series across all forecasting horizons. It is obviously and consistently that the $p \mathrm{NN}$ type models achieve the highest performance throughout all horizons from $h=1$ to 18 ( 1 month to 1.5 years). The forecasting performance of SARIMA model is significantly lower than either the $p$ NN-HP or the $p$ NN-MA model. 
Usually, in the seasonal data forecasting, the data from the same season of previous cycles (i.e., years) is more informative than that from the most recent ones. Due to the study by (Song, Smeral, G. Li, \& Chen, 2013), a 12-month lag of seasonal model in equation (2) can capture seasonal patterns well in forecasting the seasonal component of the monthly tourist arrival series. In addition, the strong capability of ARNN in capturing the non-linearity offers a significantly lower error in forecasting the trend components as the examples in Figure 5, where the Absolute Percentage Errors (APE) is $2 \%$ in (b) at the most. It is obviously that the ARNN is the key in achieving forecasting precision and underlines our contribution to the literature.

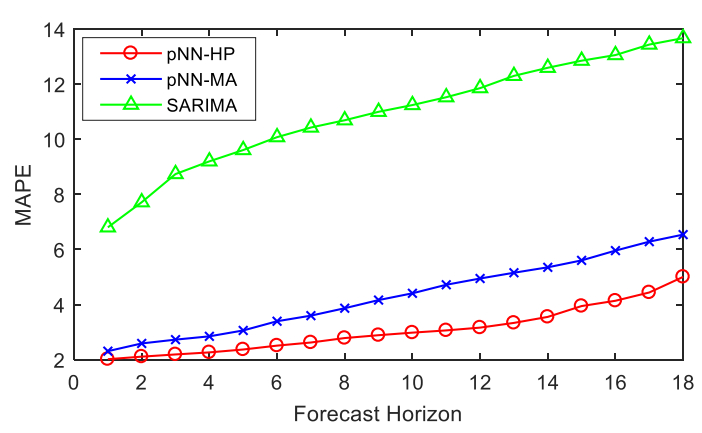

(a)

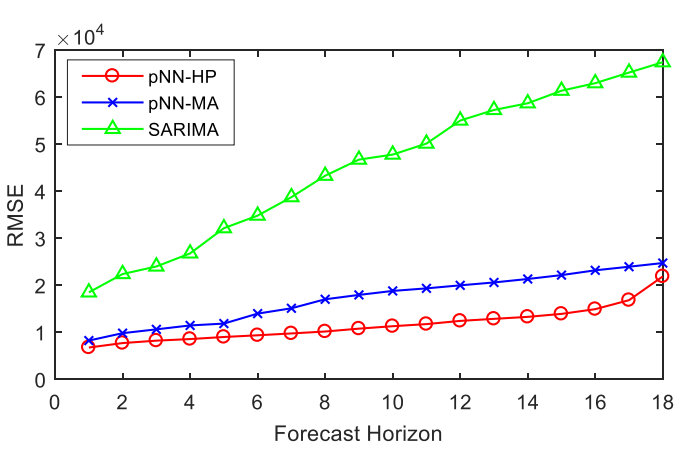

(b)

Figure 11 This figure shows the Mean Average Absolute Percentage Error (MAPE) and Root Mean Square Error (RMSE) by different horizon. The MAPE and RMSE measures are averaged across 12 market cases. The data of each single market is shown in Table 16 to Table 21. $p$ NN-HP is the paired Neural Network model with HP filter; $p$ NN-MA is the paired Neural Network with Moving Average as the trend filter; SARIMA is the Seasonal Autoregressive Integrated Moving Average.

Figure 12 provides a detailed insight into the forecast accuracy. The forecast results of horizon $h=1$ to 18 (1 month to 1.5 years) with six selected source market data including Japan, China Mainland, Canada, Mexico, France and UK are presented. As shown in Figure 12, the SARIMA model performs the worst with all data except China Mainland, where the forecast of SARIMA is the closest to the one of pNN models. We can also observe that the $p$ NN-HP model usually outperforms the $p$ NN-MA model in some examples, although in most cases they perform quite similarly. This follows our two expectations: 1) the ARNN enhances the overall performance of $p \mathrm{NN}$ type models; 2) the slightly more non-linearity of the trend by moving average than that of the trend by HP filter (as the illustration in Figure 5(b)) brings the slightly higher error rate of $p$ NN-MA than that of $p$ NN-HP, though they both achieve high accuracy compared to SARIMA model.

One of the motivation to propose $p \mathrm{NN}$ type model is to explicitly decompose the trend and the seasonal component and model and forecast them by the models that can capture the corresponding 
feature the best. ARNN is strong in capturing trend in time series (Zhang \& Qi, 2005) (Patil, Tantau, $\&$ Salokhe, 2008) as well as the cyclical patterns. Therefore, our $p$ NN type model takes advantage of the strong capability of the ARNN on the components separately. Unlike the traditional application of neural network on tourism area, which usually simply feeds the original data to the model and generates the result, our proposed $p \mathrm{NN}$ enhances the overall performance by the specifically modelling different features, which are explicitly decomposed by HP filter or moving average.

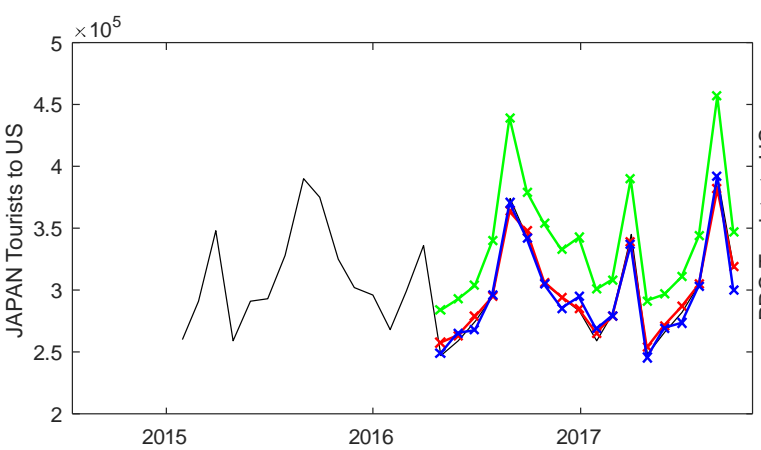

(a)

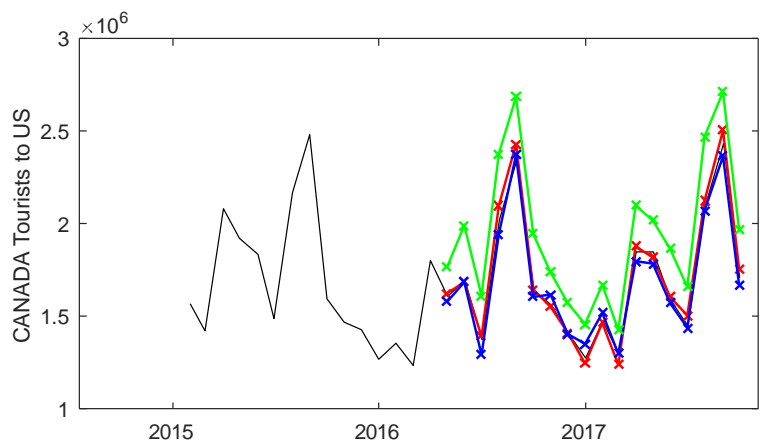

(c)

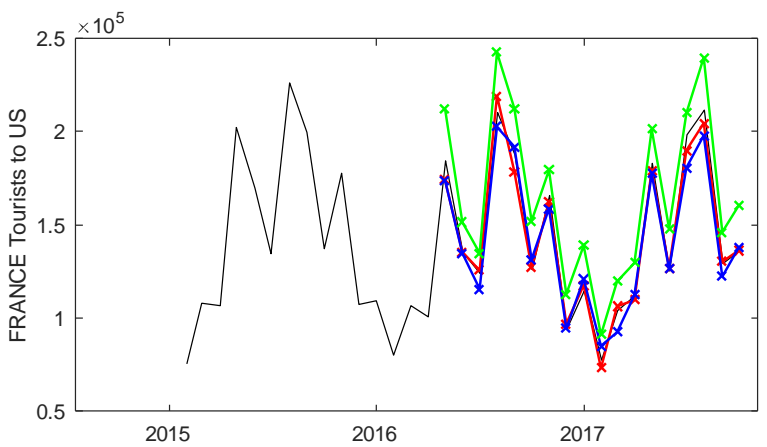

(e)

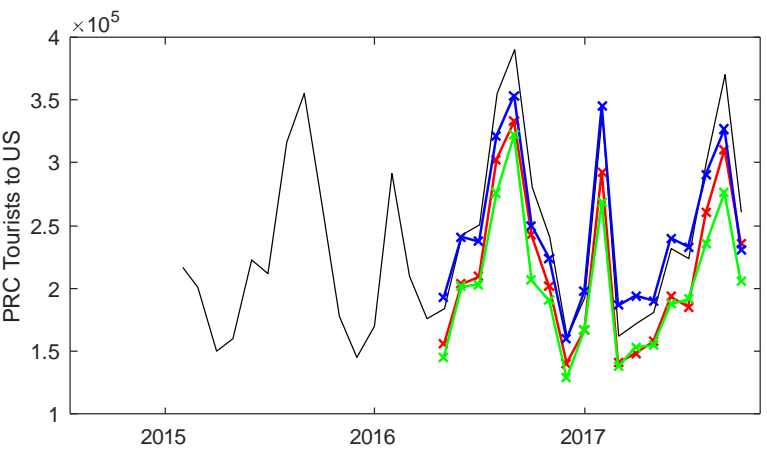

(b)

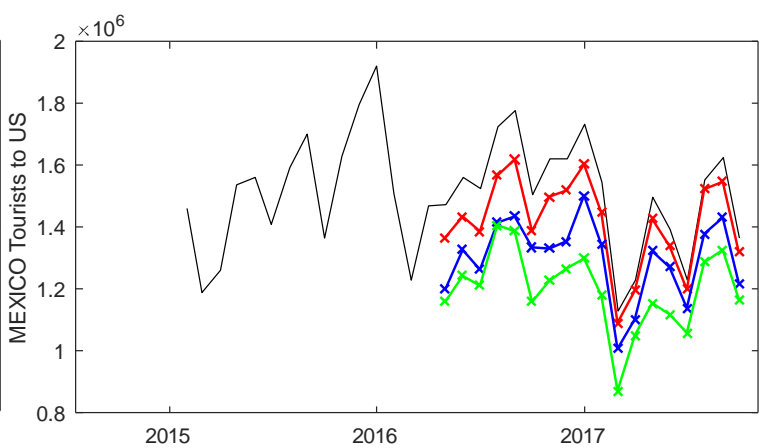

(d)

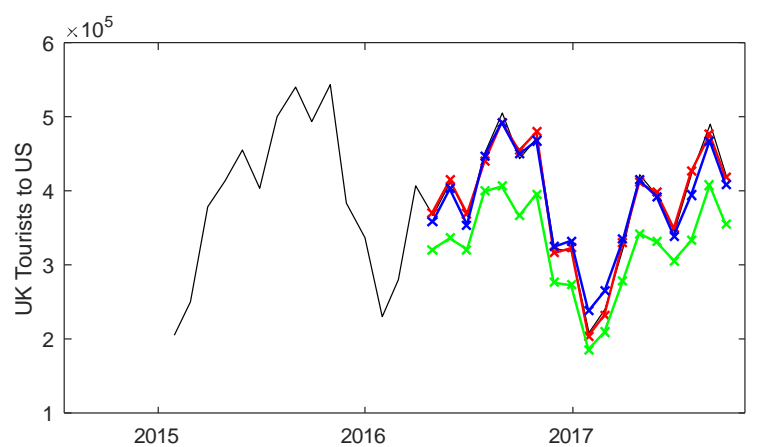

(f)

Actual Series

$*$ pNN-HP

$\longrightarrow$ pNN-MA

$\longrightarrow$ SARIMA

Figure 12 This figure shows the forecasting results of tourist arrivals from six source markets (Japan, China Mainland, Canada, Mexico, France and UK) by three models: $p$ NN-HP, $p$ NN-MA, and SARIMA. The models are estimated by the data from Jan 1996 up to Mar 2016, which is partially illustrated for the clarity of the figures. The forecasting results are up to 18 months from Apr 2016 to Sep 2017. Note: pNN-HP is the paired Neural Network with HP filter; $p$ NN-MA is the paired Neural Network model with Moving Average as the trend filter; SARIMA is the Seasonal Autoregressive Integrated Moving Average. 
In additional to the original results in Figure 12, the monthly forecast error measures of tourist arrivals data across all horizons from six source markets are shown as selected examples in Table 16 to Table 21. Even without any statistical comparison, it is clear that the $p \mathrm{NN}$ type models significantly outperform the competing model SARIMA. The performance of $p \mathrm{NN}$ type models is consistent across all horizons. However, $p \mathrm{NN}$ type models perform differently across all source market data. For example, the highest MAPE in Table 16 to Table 21 for $p$ NN-HP model is $6.2882 \%$ in tourist arrival data from China mainland. However, the lowest MAPE for $p$ NN-HP model is merely $2.6644 \%$ in Japan tourist data. We observe dramatically different patterns of the two series of Japan and China mainland in Figure 13. The tourist arrival from Japan is relatively stable except a big drop in 2001 due to the '911' event. From 2010 to 2017, the tourist arrival from Japan shows relatively similar repeated patterns. However, the tourist arrival from China mainland shows a roughly $800 \%$ increase from $0.5 \times 10^{5}$ in 1996 to $4 \times 10^{5} 2017$. From 1996 to 2008 , both the trend and seasonal components are stable. After 2008, however, the trend component shows a quick increase while the seasonal component shows an annual oscillation with divergent magnitude. Such patterns kill the precision of the autoregressive models in equation (2) and contribute to the relatively large forecasting error. We found that the average absolute percentage error (APE) of the forecast trend and seasonal components of China mainland tourist arrivals in Figure 13(a) and (b) are 2.94\% and 9.54\% respectively while the ones of Japan tourist arrivals in Figure 13(c) and (d) are 1.73\% and $4.21 \%$ respectively. Therefore, the divergent changes in seasonal component of China mainland data is the key reason for a relatively low precision in $\mathrm{pNN}$ model. However, we also notice that the tourist arrival data from China mainland to US is a special case due to the "opening up policy" in China mainland especially after 2008, when Beijing successfully hosted the Games of the XXIX Olympiad. Even though, the pNN type model still outperforms the traditional SARIMA model, which also achieves the highest MAPE of $19.7615 \%$ with China mainland tourist data.

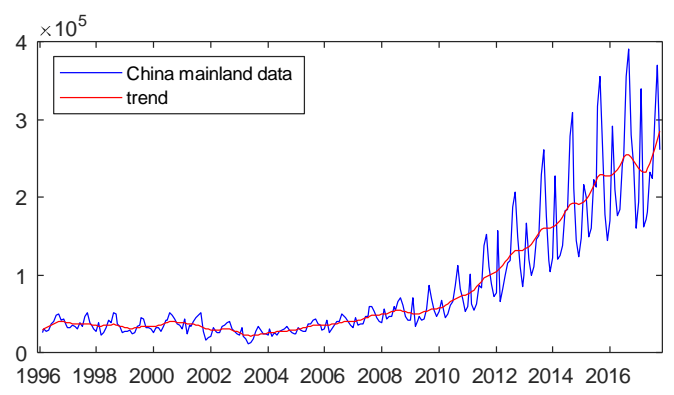

(a)

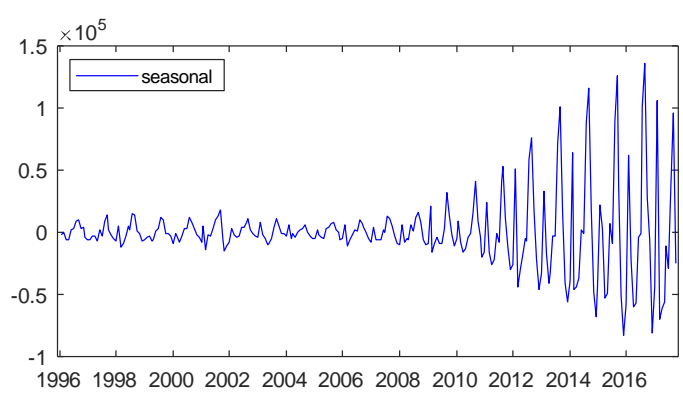

(b) 


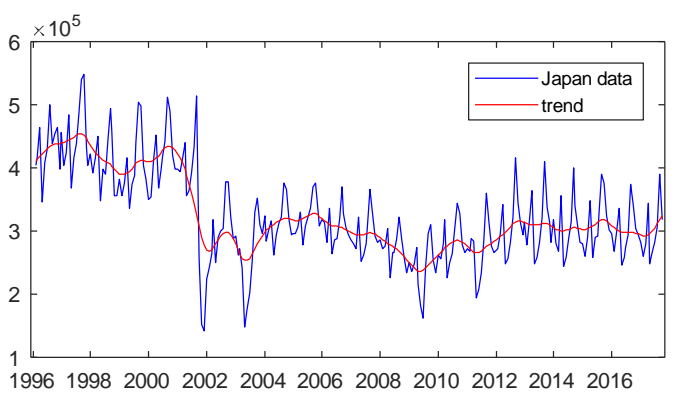

(c)

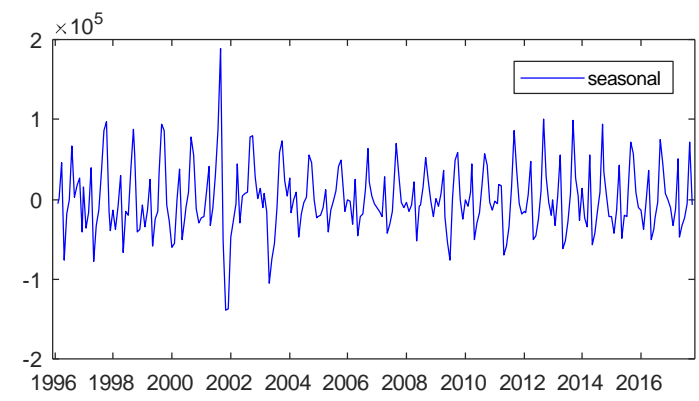

(d)

Figure 13 This figure shows a comparison of the US tourist arrival data; from Japan and China mainland. (a) the tourist arrival data from China mainland and the trend component is extracted by HP filter; (b) the seasonal component of China mainland tourist to US; (c) the tourist arrival data from Japan and the trend component is extracted by HP filter; (d) the seasonal component of Japan tourist to US.

Table 16 This table shows the average accuracy of monthly forecasts of US tourist arrivals from Japan. The MAPE and RMSE are average values across all forecasted data from Aug 2013 to Sep 2017. The models are all estimated by the data from Jan 1996 to Jul 2013.

\begin{tabular}{|c|c|c|c|c|c|c|}
\hline JAPAN & \multicolumn{3}{|c|}{ MAPE (\%) } & \multicolumn{3}{|c|}{ RMSE } \\
\hline Horizon & pNN-HP & pNN-MA & $\begin{array}{c}\text { SARIM } \\
\text { A }\end{array}$ & pNN-HP & pNN-MA & SARIMA \\
\hline 1 & 1.4181 & 2.0708 & 5.7228 & $4,054.2216$ & $5,947.8787$ & 62.8926 \\
\hline 2 & 1.4251 & 2.1153 & 6.3176 & $4,116.4230$ & $6,020.8353$ & 810.7105 \\
\hline 3 & 1.4387 & 2.2448 & 6.3184 & $4,940.8339$ & $6,933.7099$ & $2,194.7941$ \\
\hline 4 & 1.4761 & 2.2996 & 6.8054 & $4,970.5450$ & $7,182.1324$ & $2,937.8717$ \\
\hline 5 & 1.4971 & 2.4668 & 7.1076 & 4,999.1695 & $7,203.4253$ & $3,151.1202$ \\
\hline 6 & 1.6709 & 2.5655 & 7.4185 & $5,221.7649$ & $7,458.9450$ & $4,072.9544$ \\
\hline 7 & 1.6711 & 2.5902 & 7.4944 & $5,274.6463$ & $7,632.7029$ & $5,474.6889$ \\
\hline 8 & 1.9163 & 2.6175 & 7.7514 & $5,350.2330$ & $7,829.8352$ & $6,815.6600$ \\
\hline 9 & 1.9649 & 2.7587 & 7.7540 & $5,353.7903$ & $7,986.5011$ & $8,266.6955$ \\
\hline 10 & 1.9809 & 2.8478 & 8.0971 & $5,713.3339$ & $8,051.9947$ & $8,270.2917$ \\
\hline 11 & 1.9975 & 2.8738 & 8.2193 & $5,758.4859$ & $8,114.1258$ & $8,355.9751$ \\
\hline 12 & 2.0234 & 2.8808 & 8.5103 & $6,248.8423$ & $8,454.1336$ & $9,085.0629$ \\
\hline 13 & 2.2615 & 3.0187 & 8.5690 & $6,691.2576$ & $8,626.6082$ & $9,227.3149$ \\
\hline 14 & 2.2837 & 3.0473 & 8.8332 & $6,799.8298$ & $9,686.8360$ & $10,743.7732$ \\
\hline 15 & 2.3124 & 3.1867 & 8.8431 & $6,936.5144$ & $9,788.7525$ & $11,743.7681$ \\
\hline 16 & 2.3790 & 3.3717 & 8.9026 & $6,949.0603$ & $9,842.7553$ & $12,381.1422$ \\
\hline 17 & 2.6283 & 3.6827 & 9.1883 & $7,808.9009$ & $10,981.2925$ & $12,508.8093$ \\
\hline 18 & 2.6644 & 3.8045 & 9.2174 & $8,826.8160$ & $11,741.7673$ & $16,898.2777$ \\
\hline
\end{tabular}

Note: MAPE is Mean Average Absolute Percentage Error; RMSE is Root Mean Square Error; pNN-HP is the paired Neural Network model with HP filter; pNN-MA is the paired Neural Network model with Moving Average as the trend filter; SARIMA is the Seasonal Autoregressive Integrated Moving Average.

Table 17 This table shows the average accuracy of monthly forecasts of US tourist arrivals from P.R.China. The MAPE and RMSE are average values across all forecasted data from Aug 2013 to Sep 2017. The models are all estimated by the data from Jan 1996 to Jul 2013.

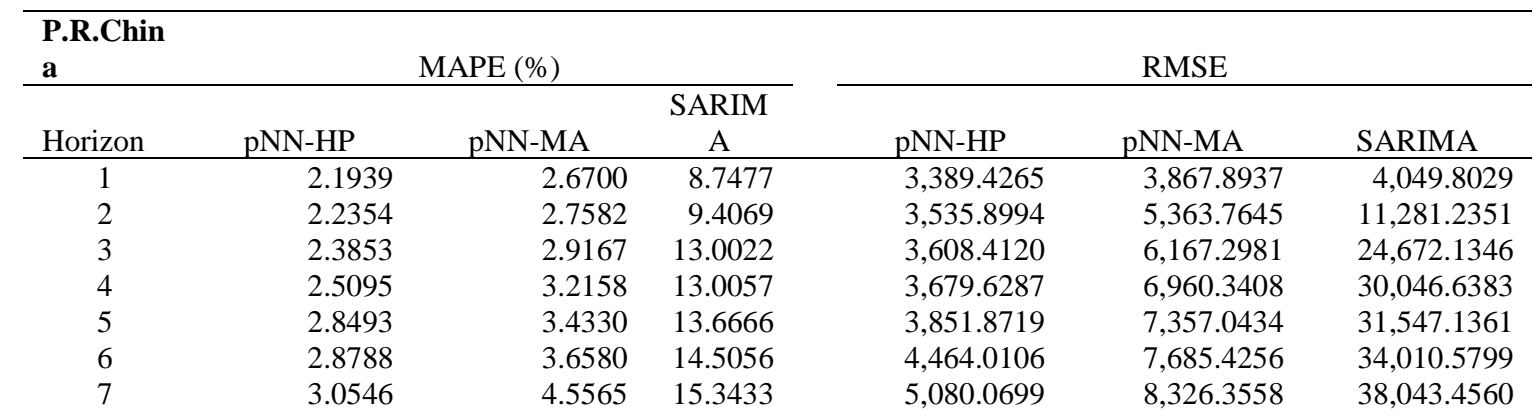




\begin{tabular}{lrrrrrr}
8 & 3.6452 & 4.9174 & 15.4939 & $5,406.6520$ & $8,777.0933$ & $41,296.2372$ \\
9 & 4.0254 & 4.9781 & 16.5549 & $5,492.1599$ & $9,142.2132$ & $44,048.4914$ \\
10 & 4.3749 & 5.0052 & 16.5588 & $5,556.7633$ & $10,562.9857$ & $44,741.0216$ \\
11 & 4.5822 & 5.5689 & 16.7300 & $6,458.7721$ & $12,049.3078$ & $47,762.3227$ \\
12 & 4.6617 & 5.6944 & 16.9114 & $9,245.5575$ & $12,343.0974$ & $52,517.3111$ \\
13 & 4.8025 & 6.1542 & 17.2186 & $9,719.7594$ & $12,744.8141$ & $55,204.0855$ \\
14 & 4.8565 & 6.8230 & 17.3124 & $10,366.2691$ & $14,315.0753$ & $62,205.1557$ \\
15 & 5.8348 & 7.6449 & 17.5534 & $12,210.8285$ & $16,494.3540$ & $62,622.7461$ \\
16 & 6.0910 & 8.7256 & 18.1220 & $13,664.4354$ & $17,081.6246$ & $63,741.2330$ \\
17 & 6.1872 & 9.1358 & 19.3662 & $15,102.8313$ & $20,231.3794$ & $73,226.0433$ \\
18 & 6.2882 & 10.2183 & 19.7615 & $15,812.8968$ & $20,998.1905$ & $77,462.0883$ \\
\hline
\end{tabular}

Note: MAPE is Mean Average Absolute Percentage Error; RMSE is Root Mean Square Error; pNN-HP is the paired Neural Network model with HP filter; pNN-MA is the paired Neural Network model with Moving Average as the trend filter; SARIMA is the Seasonal Autoregressive Integrated Moving Average.

Table 18 This table shows the average accuracy of monthly forecasts of US tourist arrivals from Canada. The MAPE and RMSE are average values across all forecasted data from Aug 2013 to Sep 2017. The models are all estimated by the data from Jan 1996 to Jul 2013.

\begin{tabular}{|c|c|c|c|c|c|c|}
\hline \multicolumn{7}{|l|}{ CANAD } \\
\hline Horizon & pNN-HP & pNN-MA & $\begin{array}{c}\text { SARIM } \\
\text { A }\end{array}$ & pNN-HP & pNN-MA & SARIMA \\
\hline 1 & 1.9035 & 2.0288 & 6.3349 & $29,574.0551$ & $29,115.2496$ & $23,612.7614$ \\
\hline 2 & 1.9213 & 2.0366 & 7.2566 & $31,639.8449$ & $32,423.3141$ & $24,007.5529$ \\
\hline 3 & 1.9998 & 2.0508 & 7.6164 & $32,997.5614$ & $33,268.1662$ & $25,359.8193$ \\
\hline 4 & 2.0176 & 2.0985 & 7.8078 & $34,349.9232$ & $37,737.8413$ & $35,513.7028$ \\
\hline 5 & 2.1590 & 2.2578 & 8.3842 & $34,652.0709$ & $38,551.6398$ & $40,877.8516$ \\
\hline 6 & 2.1766 & 2.4782 & 8.7882 & $34,838.0449$ & $40,927.5715$ & $46,406.3002$ \\
\hline 7 & 2.1797 & 2.5653 & 8.8957 & $36,389.6919$ & $46,293.5134$ & $56,914.8491$ \\
\hline 8 & 2.2958 & 2.8336 & 9.1857 & $38,467.9057$ & $53,846.6558$ & $57,658.1449$ \\
\hline 9 & 2.4023 & 2.9751 & 9.2372 & $42,776.4385$ & $54,351.7902$ & $67,248.5746$ \\
\hline 10 & 2.5649 & 3.2464 & 9.3832 & $44,424.6116$ & $55,052.8761$ & $70,715.0143$ \\
\hline 11 & 2.6107 & 3.4492 & 9.3946 & $44,483.5258$ & $56,376.6186$ & $73,552.7092$ \\
\hline 12 & 2.7096 & 3.4499 & 9.5989 & $46,719.3535$ & $59,543.0840$ & $81,403.3225$ \\
\hline 13 & 2.7706 & 3.6082 & 9.6609 & $46,869.1387$ & $59,832.5781$ & $92,093.2720$ \\
\hline 14 & 2.9847 & 3.8336 & 10.5681 & $48,633.3919$ & $61,402.9161$ & $92,634.6652$ \\
\hline 15 & 3.2304 & 4.1664 & 11.3036 & $50,546.9690$ & $62,518.2238$ & $107,550.8504$ \\
\hline 16 & 3.4763 & 4.2384 & 11.3063 & $55,964.3055$ & $66,041.3895$ & $109,282.2452$ \\
\hline 17 & 3.9160 & 4.3333 & 11.3895 & $69,943.1255$ & $66,111.7145$ & $113,239.4456$ \\
\hline 18 & 4.7323 & 4.5588 & 11.4530 & $117,251.7355$ & $70,288.0321$ & $117,940.9077$ \\
\hline
\end{tabular}

Note: MAPE is Mean Average Absolute Percentage Error; RMSE is Root Mean Square Error; pNN-HP is the paired Neural Network model with HP filter; pNN-MA is the paired Neural Network model with Moving Average as the trend filter; SARIMA is the Seasonal Autoregressive Integrated Moving Average.

Table 19 This table shows the average accuracy of monthly forecasts of US tourist arrivals from Mexico. The MAPE and RMSE are average values across all forecasted data from Aug 2013 to Sep 2017. The models are all estimated by the data from Jan 1996 to Jul 2013.

\begin{tabular}{|c|c|c|c|c|c|c|}
\hline MEXICO & \multicolumn{3}{|c|}{ MAPE $(\%)$} & \multicolumn{3}{|c|}{ RMSE } \\
\hline Horizon & pNN-HP & pNN-MA & SARIMA & pNN-HP & pNN-MA & SARIMA \\
\hline 1 & 2.0551 & 3.1615 & 8.4908 & $23,008.9286$ & $35,896.4678$ & $76,226.3350$ \\
\hline 2 & 2.1493 & 3.1794 & 11.0394 & $30,034.8306$ & $45,076.1205$ & $92,581.4979$ \\
\hline 3 & 2.2997 & 3.6235 & 12.2315 & $32,120.5691$ & $49,137.0452$ & $124,978.4799$ \\
\hline 4 & 2.3816 & 3.6724 & 13.2957 & $32,689.2784$ & $51,805.6269$ & $206,210.6986$ \\
\hline 5 & 2.3954 & 3.7165 & 13.5651 & $35,360.0412$ & $54,152.3174$ & $255,924.4047$ \\
\hline 6 & 2.4068 & 6.1008 & 14.3385 & $37,918.8539$ & $74,973.5001$ & $273,460.8150$ \\
\hline 7 & 2.5992 & 6.5296 & 14.7181 & $38,737.2888$ & $80,005.6105$ & $297,826.1855$ \\
\hline 8 & 2.6431 & 6.9635 & 14.7415 & $39,970.9958$ & $93,468.5149$ & $334,180.7186$ \\
\hline 9 & 2.6603 & 7.1458 & 14.8651 & $41,492.9709$ & $98,001.1533$ & $350,517.8578$ \\
\hline 10 & 2.7547 & 7.1770 & 15.1530 & $43,861.5262$ & $102,553.8870$ & $352,985.4123$ \\
\hline 11 & 2.9492 & 7.2857 & 15.2746 & $45,512.4265$ & $103,310.2356$ & $357,304.6869$ \\
\hline
\end{tabular}




$\begin{array}{lllllll}12 & 2.9575 & 7.3130 & 15.6239 & 46,122.7767 & 105,057.1264 & 392,696.8724 \\ 13 & 3.0117 & 7.7906 & 15.8549 & 46,799.1201 & 105,735.4548 & 402,176.7511 \\ 14 & 3.1128 & 7.8629 & 16.3022 & 47,804.7792 & 107,459.8577 & 406,559.9869 \\ 15 & 3.2467 & 7.8649 & 16.5410 & 48,535.5247 & 110,370.2890 & 415,238.6482 \\ 16 & 3.3036 & 8.1077 & 16.7006 & 49,214.1087 & 114,738.5518 & 419,592.4298 \\ 17 & 3.5934 & 8.1079 & 16.7132 & 50,539.1630 & 116,850.5917 & 424,200.9279 \\ 18 & 3.6229 & 8.1335 & 16.9801 & 54,686.8468 & 119,028.1019 & 430,970.4110\end{array}$

Note: MAPE is Mean Average Absolute Percentage Error; RMSE is Root Mean Square Error; pNN-HP is the paired Neural Network model with HP filter; pNN-MA is the paired Neural Network model with Moving Average as the trend filter; SARIMA is the Seasonal Autoregressive Integrated Moving Average.

Table 20 This table shows the average accuracy of monthly forecasts of US tourist arrivals from FRANCE. The MAPE and RMSE are average values across all forecasted data from Aug 2013 to Sep 2017. The models are all estimated by the data from Jan 1996 to Jul 2013.

\begin{tabular}{|c|c|c|c|c|c|c|}
\hline FRANCE & \multicolumn{3}{|c|}{ MAPE (\%) } & \multicolumn{3}{|c|}{ RMSE } \\
\hline Horizon & pNN-HP & pNN-MA & $\begin{array}{c}\text { SARIM } \\
\text { A }\end{array}$ & pNN-HP & pNN-MA & SARIMA \\
\hline 1 & 2.0968 & 2.6193 & 8.1461 & $2,204.1445$ & $2,583.0345$ & $2,787.1725$ \\
\hline 2 & 2.1712 & 3.3467 & 9.1114 & $2,321.2121$ & $3,559.6996$ & $5,871.9584$ \\
\hline 3 & 2.2151 & 3.6270 & 10.7600 & $2,441.6981$ & $4,545.7766$ & $14,876.8553$ \\
\hline 4 & 2.2767 & 3.7776 & 11.3432 & $2,478.8577$ & $4,770.9112$ & $16,543.2730$ \\
\hline 5 & 2.3220 & 5.2181 & 11.4788 & $3,017.5703$ & $4,847.1334$ & $17,364.3711$ \\
\hline 6 & 2.5546 & 5.3940 & 12.2371 & $3,054.1489$ & $5,440.8341$ & $18,519.9672$ \\
\hline 7 & 2.6189 & 5.4629 & 12.3979 & $3,187.0396$ & $5,883.8394$ & $20,226.9403$ \\
\hline 8 & 2.7427 & 5.6700 & 12.6864 & $3,240.0692$ & $6,090.0635$ & $22,121.0034$ \\
\hline 9 & 2.8423 & 5.7421 & 13.1152 & $3,460.5369$ & $6,735.7209$ & $24,832.6736$ \\
\hline 10 & 2.8521 & 5.7862 & 13.5528 & $3,502.2232$ & $7,284.6118$ & $25,671.8729$ \\
\hline 11 & 3.0307 & 6.2567 & 14.0838 & $3,546.2541$ & $8,493.6410$ & $27,960.8082$ \\
\hline 12 & 3.2129 & 6.3154 & 14.6017 & $4,547.2265$ & $8,643.9093$ & $28,581.9237$ \\
\hline 13 & 3.2134 & 6.3256 & 15.7236 & $4,788.6029$ & $9,078.5282$ & $28,688.1238$ \\
\hline 14 & 3.4172 & 6.9927 & 15.7812 & $4,809.2516$ & $10,274.5840$ & $29,176.0713$ \\
\hline 15 & 3.5129 & 7.0585 & 15.8486 & $5,351.4803$ & $10,315.5725$ & $30,310.6195$ \\
\hline 16 & 3.6808 & 8.1430 & 15.8984 & $5,949.9461$ & $10,368.8115$ & $31,245.8847$ \\
\hline 17 & 3.8886 & 8.2036 & 16.0508 & $6,418.1043$ & $10,462.3922$ & $32,360.6636$ \\
\hline 18 & 4.0212 & 8.2543 & 16.1345 & $6,530.2179$ & $10,671.9451$ & $32,633.9539$ \\
\hline
\end{tabular}

Note: MAPE is Mean Average Absolute Percentage Error; RMSE is Root Mean Square Error; pNN-HP is the paired Neural Network model with HP filter; pNN-MA is the paired Neural Network model with Moving Average as the trend filter; SARIMA is the Seasonal Autoregressive Integrated Moving Average.

Table 21 This table shows the average accuracy of monthly forecasts of US tourist arrivals from UK. The MAPE and RMSE are average values across all forecasted data from Aug 2013 to Sep 2017. The models are all estimated by the data from Jan 1996 to Jul 2013.

\begin{tabular}{|c|c|c|c|c|c|c|}
\hline UK & \multicolumn{3}{|c|}{ MAPE (\%) } & \multicolumn{3}{|c|}{ RMSE } \\
\hline Horizon & pNN-HP & pNN-MA & $\begin{array}{c}\text { SARIM } \\
\text { A }\end{array}$ & pNN-HP & pNN-MA & SARIMA \\
\hline 1 & 2.0125 & 2.0136 & 6.0649 & $5,905.0835$ & $6,811.1439$ & 365.4765 \\
\hline 2 & 2.0292 & 2.0192 & 6.2400 & $6,394.6941$ & $7,184.9834$ & 459.0899 \\
\hline 3 & 2.1592 & 2.0834 & 6.8939 & $7,055.4306$ & $8,102.3625$ & $2,735.7885$ \\
\hline 4 & 2.1748 & 2.1805 & 7.6881 & $7,914.8472$ & $8,925.2220$ & $4,089.6066$ \\
\hline 5 & 2.3482 & 2.2493 & 8.0631 & $8,553.7269$ & $9,114.8483$ & $4,516.5265$ \\
\hline 6 & 2.6110 & 2.4806 & 8.2551 & $8,553.8564$ & $9,160.5909$ & $4,824.4235$ \\
\hline 7 & 2.6127 & 2.6586 & 8.7022 & $9,470.1553$ & $9,992.9597$ & $6,131.5844$ \\
\hline 8 & 2.7743 & 2.9626 & 8.8264 & $9,484.3761$ & $10,021.0201$ & $8,507.2936$ \\
\hline 9 & 2.8075 & 3.0014 & 8.9665 & $9,522.9954$ & $10,109.8395$ & $10,287.9342$ \\
\hline 10 & 2.8297 & 3.7035 & 9.0428 & $10,405.7139$ & $11,812.6566$ & $11,472.7231$ \\
\hline 11 & 2.8436 & 3.8187 & 9.1872 & $10,583.7699$ & $11,866.8348$ & $15,061.4749$ \\
\hline 12 & 3.1550 & 3.9078 & 9.2531 & $10,797.7889$ & $12,275.2218$ & $15,151.2231$ \\
\hline 13 & 3.5197 & 4.0512 & 9.8970 & $12,552.6999$ & $12,801.4114$ & $16,132.8664$ \\
\hline 14 & 3.7874 & 4.1160 & 9.9473 & $12,601.5293$ & $13,098.4995$ & $16,573.6554$ \\
\hline 15 & 3.9738 & 4.2072 & 10.2926 & $13,862.9409$ & $14,147.0197$ & $16,864.2814$ \\
\hline
\end{tabular}




\begin{tabular}{lllllll}
16 & 4.0694 & 4.2164 & 10.2984 & $14,115.8330$ & $15,423.6550$ & $22,046.4316$ \\
17 & 4.3471 & 5.1342 & 10.6998 & $16,254.6417$ & $16,217.2473$ & $22,457.6025$ \\
18 & 5.2968 & 5.6525 & 10.7351 & $16,629.8387$ & $16,722.6244$ & $23,874.5622$ \\
\hline
\end{tabular}

Note: MAPE is Mean Average Absolute Percentage Error; RMSE is Root Mean Square Error; pNN-HP is the paired Neural Network model with HP filter; pNN-MA is the paired Neural Network model with Moving Average as the trend filter; SARIMA is the Seasonal Autoregressive Integrated Moving Average.

\section{Conclusion}

The fact that tourism data comprises both a long-term trend component and a strong repeated seasonal component has crucial implications for modelling and forecasting tourism data over both short and long horizons. In this paper, we combine the ideas from the work in (Harvey \& Todd, 1983), (Chen, $\mathrm{Li}, \mathrm{Wu}, \&$ Shen, 2017) and (Yao, et al., 2017) to propose a novel paired computational model. The original tourist arrival data is explicitly decomposed by the low-pass Hodrick-Prescott filter, wavelet transformation and a simple moving average method subject to the stationarity and seasonality of the seasonal component, which is therefore guaranteed to be a stationary seasonal process. The trend component is then obtained by subtracting the seasonal process from the original tourism series. Two separated three-layer autoregressive neural network are then estimated by the trend and seasonal component data respectively. The outputs of the two autoregressive neural networks are then aggregated as the final forecasting result of the tourism series. The three models are termed as paired Neural Network with HP filter (pNN-HP), paired Neural network with Wavelet Transformation (pNN-WT) and with Moving Average (pNN-MA). The models were thoroughly evaluated using the tourist arrival data to US from top 12 source markets up to 18 forecasting horizons. The out-ofsample forecasting results of pNN type models consistently and significantly outperform the traditional ARIMA, ARFIMA, and SARIMA model.

Previous studies of tourism arrival forecasting show that incorporating macro-economic data, i.e., FX rate, GDP growth rate, consumer price index (CPI), and others, into the model may increase the forecasting accuracy. This has not been thoroughly studied in computational models. One of the future directions may investigate the impact of incorporating such macro-economic factors into our proposed method as an exogenous feature for either trend or seasonal component modelling. Another future direction may fall in the optimal public resource allocation according to the forecasted tourist arrival. Further investigation may also consider using the pNN model in other areas which can also be observed with trend and oscillation components. 


\section{Acknowledgements}

This research is partially supported by the National Social Science Fund of China (Grant No. 17BJY194), Scientific Research Funds for the Returned Overseas Chinese Scholars from State Education Ministry, the Funds for Young Key Program of Education Department from Fujian Province, China (Grant No. JZ160425 ), Program of Education Department of Fujian Province, China (Grant No. I201501005 ), and Nature and Science Funds from Fujian Province, China (Grant No. 2015J01236 ).

\section{Appendix}

Appendix Table 1 This table shows the accuracy of monthly forecasts of US tourist arrivals from Italy. The Absolute Percentage Error (APE) is calculated at forecasting horizon 1 to 18 months (1.5 years). The $p$ NN-HP, $p$ NN-WT and $p$ NNMA models are all estimated by the data from Jan 1996 to Jun 2013 (210 months).

\begin{tabular}{|c|c|c|c|c|c|c|c|c|c|}
\hline \multirow[b]{2}{*}{ horizon } & \multicolumn{4}{|c|}{$p$ NN HP } & \multicolumn{4}{|c|}{$p$ NN WT } & \multirow{2}{*}{$p$ NN MA } \\
\hline & 100 & 1600 & 3600 & 129600 & 3 & 4 & 5 & 6 & \\
\hline 1 & $1.8834 \%$ & $3.6798 \%$ & $3.2606 \%$ & $3.2447 \%$ & $1.9165 \%$ & $3.8966 \%$ & $3.6859 \%$ & $3.8178 \%$ & $7.6585 \%$ \\
\hline 2 & $3.2969 \%$ & $3.7767 \%$ & $3.2925 \%$ & $4.4096 \%$ & $2.9805 \%$ & $5.6386 \%$ & $4.9472 \%$ & $4.3655 \%$ & $3.1673 \%$ \\
\hline 3 & $2.3359 \%$ & $3.3024 \%$ & $2.7045 \%$ & $3.9159 \%$ & $1.1998 \%$ & $3.4319 \%$ & $1.5650 \%$ & $2.5394 \%$ & $4.2601 \%$ \\
\hline 4 & $2.6379 \%$ & $3.9657 \%$ & $2.6882 \%$ & $3.5342 \%$ & $1.7236 \%$ & $2.0981 \%$ & $2.7083 \%$ & $2.3921 \%$ & $8.2053 \%$ \\
\hline 5 & $3.6337 \%$ & $3.7852 \%$ & $2.3624 \%$ & $2.9708 \%$ & $4.1219 \%$ & $1.4097 \%$ & $2.7324 \%$ & $2.1635 \%$ & $8.5099 \%$ \\
\hline 6 & $2.3655 \%$ & $3.0335 \%$ & $2.0080 \%$ & $3.0083 \%$ & $0.2408 \%$ & $1.8884 \%$ & $1.9412 \%$ & $2.2997 \%$ & $0.6997 \%$ \\
\hline 7 & $4.0520 \%$ & $3.6339 \%$ & $2.1511 \%$ & $3.7926 \%$ & $2.1551 \%$ & $1.3079 \%$ & $3.0249 \%$ & $2.4192 \%$ & $1.2377 \%$ \\
\hline 8 & $4.9458 \%$ & $3.0138 \%$ & $2.2946 \%$ & $3.1112 \%$ & $2.7041 \%$ & $1.9558 \%$ & $0.4935 \%$ & $2.6845 \%$ & $4.2999 \%$ \\
\hline 9 & $3.6619 \%$ & $3.7592 \%$ & $3.8218 \%$ & $3.6830 \%$ & $3.2888 \%$ & $3.7332 \%$ & $3.8716 \%$ & $3.1956 \%$ & $5.2757 \%$ \\
\hline 10 & $4.6232 \%$ & $4.5893 \%$ & $4.4245 \%$ & $4.4238 \%$ & $2.4133 \%$ & $4.8403 \%$ & $5.0526 \%$ & $4.0637 \%$ & $2.4378 \%$ \\
\hline 11 & $4.8412 \%$ & $4.8339 \%$ & $4.9444 \%$ & $4.0133 \%$ & $3.9004 \%$ & $4.7999 \%$ & $2.8913 \%$ & $4.0298 \%$ & $0.7851 \%$ \\
\hline 12 & $4.5472 \%$ & $4.3647 \%$ & $4.2717 \%$ & $4.0503 \%$ & $4.5972 \%$ & $4.1568 \%$ & $4.1227 \%$ & $4.9660 \%$ & $3.8852 \%$ \\
\hline 13 & $6.7218 \%$ & $7.6744 \%$ & $8.1897 \%$ & $6.9092 \%$ & $5.5422 \%$ & $7.2086 \%$ & $8.0285 \%$ & $7.0527 \%$ & $9.1671 \%$ \\
\hline 14 & $6.7769 \%$ & $8.4618 \%$ & $9.0115 \%$ & $8.7351 \%$ & $7.4677 \%$ & $8.2516 \%$ & $8.6577 \%$ & $7.5335 \%$ & $3.9136 \%$ \\
\hline 15 & $5.8563 \%$ & $8.9223 \%$ & $7.2624 \%$ & $8.3574 \%$ & $5.4409 \%$ & $6.8612 \%$ & $5.0276 \%$ & $7.4879 \%$ & $5.5098 \%$ \\
\hline 16 & $6.5142 \%$ & $8.2216 \%$ & $7.0357 \%$ & $8.8114 \%$ & $6.6759 \%$ & $5.9738 \%$ & $5.4662 \%$ & $7.5408 \%$ & $10.7518 \%$ \\
\hline 17 & $6.1728 \%$ & $8.6735 \%$ & $7.4877 \%$ & $8.0234 \%$ & $8.7274 \%$ & $5.4380 \%$ & $5.1129 \%$ & $7.9598 \%$ & $13.5064 \%$ \\
\hline 18 & $6.4737 \%$ & $8.7315 \%$ & $7.7047 \%$ & $8.6907 \%$ & $5.5002 \%$ & $6.4083 \%$ & $6.1162 \%$ & $7.8750 \%$ & $4.4339 \%$ \\
\hline Average & $4.5189 \%$ & $5.3568 \%$ & $4.7176 \%$ & $5.2047 \%$ & $3.9220 \%$ & $4.4055 \%$ & $4.1914 \%$ & $4.6881 \%$ & $5.4280 \%$ \\
\hline
\end{tabular}

Appendix Table 2 This table shows the accuracy of monthly forecasts of US tourist arrivals from Italy. The Absolute Percentage Error (APE) is calculated at forecasting horizon 1 to 18 months (1.5 years). The ARFIMA, ARIMA, and SARIMA models are all estimated by the data from Jan 1996 to Jun 2013 (210 months).

\begin{tabular}{rrrr}
\hline horizon & ARFIMA & ARIMA & SARIMA \\
\hline 1 & $4.8337 \%$ & $2.9845 \%$ & $3.7147 \%$ \\
2 & $7.3305 \%$ & $4.0975 \%$ & $4.3003 \%$ \\
3 & $9.3149 \%$ & $4.8629 \%$ & $3.5848 \%$
\end{tabular}




\begin{tabular}{rrrr}
4 & $10.5899 \%$ & $6.2805 \%$ & $4.2567 \%$ \\
5 & $11.2942 \%$ & $7.3904 \%$ & $4.5794 \%$ \\
6 & $12.9230 \%$ & $9.8283 \%$ & $3.6578 \%$ \\
7 & $13.1310 \%$ & $11.5981 \%$ & $4.4094 \%$ \\
8 & $17.1081 \%$ & $21.0395 \%$ & $5.7864 \%$ \\
9 & $20.3993 \%$ & $23.2992 \%$ & $7.0899 \%$ \\
10 & $21.5531 \%$ & $25.4982 \%$ & $7.6291 \%$ \\
11 & $24.7724 \%$ & $25.8667 \%$ & $7.7889 \%$ \\
12 & $30.4643 \%$ & $28.6646 \%$ & $8.9173 \%$ \\
13 & $30.5109 \%$ & $31.4510 \%$ & $11.6778 \%$ \\
14 & $30.6875 \%$ & $35.5084 \%$ & $12.2732 \%$ \\
15 & $71.7278 \%$ & $70.0575 \%$ & $18.4101 \%$ \\
16 & $73.4550 \%$ & $76.5076 \%$ & $19.7231 \%$ \\
17 & $76.6729 \%$ & $87.5999 \%$ & $21.3977 \%$ \\
18 & $78.1498 \%$ & $105.2109 \%$ & $22.2995 \%$ \\
\hline Average & $30.2732 \%$ & $32.0970 \%$ & $9.5276 \%$ \\
\hline
\end{tabular}

Appendix Table 3 This table shows the accuracy of monthly forecasts of US tourist arrivals from Spain. The Absolute Percentage Error (APE) is calculated at forecasting horizon 1 to 18 months (1.5 years). The $p$ NN-HP, $p$ NN-WT and $p$ NNMA models are all estimated by the data from Jan 1996 to Jun 2013 (210 months).

\begin{tabular}{|c|c|c|c|c|c|c|c|c|c|}
\hline \multirow[b]{2}{*}{ horizon } & \multicolumn{4}{|c|}{$p$ NN HP } & \multicolumn{4}{|c|}{$p$ NN WT } & \multirow{2}{*}{$p$ NN MA } \\
\hline & 100 & 1600 & 3600 & 129600 & 3 & 4 & 5 & 6 & \\
\hline 1 & $3.9404 \%$ & $1.8678 \%$ & $3.4009 \%$ & $3.2953 \%$ & $0.3384 \%$ & $2.9523 \%$ & $5.3060 \%$ & $3.2152 \%$ & $11.4071 \%$ \\
\hline 2 & $0.4436 \%$ & $3.5890 \%$ & $3.7500 \%$ & $4.8361 \%$ & $2.1817 \%$ & $4.0248 \%$ & $4.6438 \%$ & $4.1263 \%$ & $3.9653 \%$ \\
\hline 3 & $2.2396 \%$ & $1.5545 \%$ & $1.4217 \%$ & $1.7788 \%$ & $2.3409 \%$ & $2.4067 \%$ & $1.5814 \%$ & $1.6889 \%$ & $2.0364 \%$ \\
\hline 4 & $2.6049 \%$ & $1.9242 \%$ & $1.8057 \%$ & $2.2602 \%$ & $2.2467 \%$ & $2.9203 \%$ & $2.0785 \%$ & $1.5532 \%$ & $6.0988 \%$ \\
\hline 5 & $1.6320 \%$ & $1.6675 \%$ & $1.0553 \%$ & $1.2478 \%$ & $3.4057 \%$ & $2.7812 \%$ & $2.7180 \%$ & $2.3709 \%$ & $7.3967 \%$ \\
\hline 6 & $2.5783 \%$ & $2.5089 \%$ & $2.4942 \%$ & $2.2306 \%$ & $2.8332 \%$ & $2.5722 \%$ & $2.6292 \%$ & $3.6606 \%$ & $1.2601 \%$ \\
\hline 7 & $2.2083 \%$ & $1.8717 \%$ & $1.6733 \%$ & $3.4554 \%$ & $3.3297 \%$ & $2.2537 \%$ & $1.7293 \%$ & $3.2302 \%$ & $1.0903 \%$ \\
\hline 8 & $4.1944 \%$ & $3.9225 \%$ & $3.8820 \%$ & $3.8851 \%$ & $4.3880 \%$ & $2.0576 \%$ & $3.9993 \%$ & $3.4900 \%$ & $3.4002 \%$ \\
\hline 9 & $3.5175 \%$ & $3.3679 \%$ & $3.3633 \%$ & $3.1606 \%$ & $2.9070 \%$ & $3.2828 \%$ & $3.5249 \%$ & $3.6301 \%$ & $6.6141 \%$ \\
\hline 10 & $3.8610 \%$ & $4.2484 \%$ & $4.1241 \%$ & $4.5263 \%$ & $4.8015 \%$ & $4.1200 \%$ & $4.3826 \%$ & $3.8609 \%$ & $5.4475 \%$ \\
\hline 11 & $3.3202 \%$ & $3.4834 \%$ & $3.1153 \%$ & $3.2486 \%$ & $4.1161 \%$ & $3.4901 \%$ & $3.3317 \%$ & $3.8897 \%$ & $2.4664 \%$ \\
\hline 12 & $3.8241 \%$ & $3.8620 \%$ & $3.7845 \%$ & $4.6260 \%$ & $4.4709 \%$ & $3.9162 \%$ & $4.4794 \%$ & $3.8702 \%$ & $6.9459 \%$ \\
\hline 13 & $7.8125 \%$ & $7.5381 \%$ & $8.0174 \%$ & $9.2755 \%$ & $5.5975 \%$ & $7.1380 \%$ & $8.2116 \%$ & $6.6697 \%$ & $14.2789 \%$ \\
\hline 14 & $7.7191 \%$ & $9.1000 \%$ & $7.9996 \%$ & $9.8795 \%$ & $7.2804 \%$ & $8.9833 \%$ & $9.0668 \%$ & $6.9508 \%$ & $3.0170 \%$ \\
\hline 15 & $5.8960 \%$ & $5.9729 \%$ & $5.9810 \%$ & $6.2228 \%$ & $5.3190 \%$ & $5.9420 \%$ & $6.1545 \%$ & $6.1454 \%$ & $6.6676 \%$ \\
\hline 16 & $6.2777 \%$ & $5.8600 \%$ & $5.7269 \%$ & $6.9562 \%$ & $6.5634 \%$ & $5.9733 \%$ & $6.8173 \%$ & $6.4820 \%$ & $6.1808 \%$ \\
\hline 17 & $5.9285 \%$ & $5.7847 \%$ & $5.7023 \%$ & $7.2565 \%$ & $6.4551 \%$ & $7.1493 \%$ & $6.6517 \%$ & $6.3988 \%$ & $9.4911 \%$ \\
\hline 18 & $6.7653 \%$ & $6.4748 \%$ & $6.6301 \%$ & $6.7869 \%$ & $7.5596 \%$ & $6.4853 \%$ & $6.6768 \%$ & $6.1579 \%$ & $5.5296 \%$ \\
\hline Average & $4.1535 \%$ & $4.1444 \%$ & $4.1071 \%$ & $4.7182 \%$ & $4.2297 \%$ & $4.3583 \%$ & $4.6657 \%$ & $4.2995 \%$ & $5.7385 \%$ \\
\hline
\end{tabular}


Appendix Table 4 This table shows the accuracy of monthly forecasts of US tourist arrivals from Spain. The Absolute Percentage Error (APE) is calculated at forecasting horizon 1 to 18 months (1.5 years). The ARFIMA, ARIMA, and SARIMA models are all estimated by the data from Jan 1996 to Jun 2013 (210 months).

\begin{tabular}{rrrr}
\hline horizon & ARFIMA & ARIMA & SARIMA \\
\hline 1 & $4.8337 \%$ & $2.9845 \%$ & $3.9583 \%$ \\
2 & $7.3305 \%$ & $4.0975 \%$ & $3.9080 \%$ \\
3 & $9.3149 \%$ & $4.8629 \%$ & $2.8388 \%$ \\
4 & $10.5899 \%$ & $6.2805 \%$ & $3.6694 \%$ \\
5 & $11.2942 \%$ & $7.3904 \%$ & $3.9054 \%$ \\
6 & $12.9230 \%$ & $9.8283 \%$ & $4.1381 \%$ \\
7 & $13.1310 \%$ & $11.5981 \%$ & $4.1428 \%$ \\
8 & $17.1081 \%$ & $21.0395 \%$ & $6.4879 \%$ \\
9 & $20.3993 \%$ & $23.2992 \%$ & $7.0061 \%$ \\
10 & $21.5531 \%$ & $25.4982 \%$ & $7.8567 \%$ \\
11 & $24.7724 \%$ & $25.8667 \%$ & $7.3728 \%$ \\
12 & $30.4643 \%$ & $28.6646 \%$ & $8.9916 \%$ \\
13 & $30.5109 \%$ & $31.4510 \%$ & $12.4092 \%$ \\
14 & $30.6875 \%$ & $35.5084 \%$ & $12.3811 \%$ \\
15 & $71.7278 \%$ & $70.0575 \%$ & $17.8260 \%$ \\
16 & $73.4550 \%$ & $76.5076 \%$ & $18.8000 \%$ \\
17 & $76.6729 \%$ & $87.5999 \%$ & $20.4628 \%$ \\
18 & $78.1498 \%$ & $105.2109 \%$ & $22.0388 \%$ \\
\hline Average & $30.2732 \%$ & $18.7821 \%$ & $8.1337 \%$ \\
\hline & & &
\end{tabular}

Appendix Table 5 This table shows the accuracy of monthly forecasts of US tourist arrivals from Korea. The Absolute Percentage Error (APE) is calculated at forecasting horizon 1 to 18 months (1.5 years). The $p$ NN-HP, $p$ NN-WT and $p$ NNMA models are all estimated by the data from Jan 1996 to Jun 2013 (210 months).

\begin{tabular}{rrrrr|rrrr|c}
\hline \multicolumn{7}{c}{$p$} & \multicolumn{4}{c|}{$p$ NN WT } & \multirow{2}{*}{$p$ NN } \\
\cline { 1 - 7 } horizon & 100 & 1600 & 3600 & 129600 & 3 & 4 & 5 & 6 & MA \\
\hline 1 & $1.4833 \%$ & $1.6130 \%$ & $1.1895 \%$ & $1.6361 \%$ & $2.3102 \%$ & $1.4467 \%$ & $1.1165 \%$ & $1.6372 \%$ & $0.3993 \%$ \\
2 & $0.5696 \%$ & $0.9445 \%$ & $1.7017 \%$ & $0.8949 \%$ & $1.6163 \%$ & $1.7240 \%$ & $1.0454 \%$ & $0.9446 \%$ & $0.4773 \%$ \\
3 & $1.4650 \%$ & $2.0983 \%$ & $2.5702 \%$ & $1.9674 \%$ & $1.5572 \%$ & $2.2340 \%$ & $1.5369 \%$ & $1.9706 \%$ & $0.8352 \%$ \\
4 & $0.5609 \%$ & $2.5090 \%$ & $2.6373 \%$ & $2.2324 \%$ & $1.5117 \%$ & $2.3183 \%$ & $1.9413 \%$ & $1.7774 \%$ & $0.0423 \%$ \\
5 & $1.3050 \%$ & $1.9494 \%$ & $1.7271 \%$ & $1.7468 \%$ & $2.9070 \%$ & $1.8633 \%$ & $1.1735 \%$ & $1.6621 \%$ & $0.7927 \%$ \\
6 & $0.8244 \%$ & $1.6610 \%$ & $1.5431 \%$ & $1.3776 \%$ & $1.0674 \%$ & $1.5622 \%$ & $2.8020 \%$ & $1.0367 \%$ & $0.6124 \%$ \\
7 & $2.3906 \%$ & $3.1946 \%$ & $2.7689 \%$ & $2.9404 \%$ & $2.2155 \%$ & $3.2558 \%$ & $2.5712 \%$ & $3.1541 \%$ & $1.9329 \%$ \\
8 & $1.1565 \%$ & $1.6484 \%$ & $2.6487 \%$ & $2.0991 \%$ & $1.9205 \%$ & $2.0579 \%$ & $2.5291 \%$ & $3.7531 \%$ & $1.3706 \%$ \\
9 & $0.8663 \%$ & $2.7252 \%$ & $2.7978 \%$ & $1.9899 \%$ & $2.8930 \%$ & $2.6903 \%$ & $2.1904 \%$ & $3.2426 \%$ & $0.5273 \%$ \\
10 & $1.2431 \%$ & $3.2697 \%$ & $2.9075 \%$ & $2.2776 \%$ & $2.9132 \%$ & $3.1042 \%$ & $3.6356 \%$ & $3.1967 \%$ & $1.1658 \%$ \\
11 & $0.8030 \%$ & $2.9149 \%$ & $2.5571 \%$ & $1.7552 \%$ & $3.1790 \%$ & $3.7770 \%$ & $4.2265 \%$ & $4.9459 \%$ & $0.6421 \%$ \\
12 & $1.2915 \%$ & $3.4126 \%$ & $2.9361 \%$ & $2.0615 \%$ & $3.1543 \%$ & $3.8737 \%$ & $4.9804 \%$ & $4.0803 \%$ & $1.9029 \%$ \\
13 & $3.3155 \%$ & $5.2322 \%$ & $3.6473 \%$ & $3.7505 \%$ & $5.2981 \%$ & $5.9454 \%$ & $5.5485 \%$ & $5.8815 \%$ & $3.0084 \%$ \\
14 & $3.0537 \%$ & $6.3055 \%$ & $5.0109 \%$ & $3.4733 \%$ & $5.9787 \%$ & $6.2012 \%$ & $5.2038 \%$ & $5.8713 \%$ & $3.8510 \%$ \\
15 & $3.2099 \%$ & $6.3286 \%$ & $5.4047 \%$ & $3.2177 \%$ & $5.7342 \%$ & $6.2115 \%$ & $6.2974 \%$ & $6.3826 \%$ & $2.3703 \%$
\end{tabular}




\begin{tabular}{rrrrr|rrrr|r}
16 & $3.1784 \%$ & $5.9957 \%$ & $5.1723 \%$ & $2.9024 \%$ & $5.9518 \%$ & $6.0867 \%$ & $6.1833 \%$ & $6.5323 \%$ & $3.5323 \%$ \\
17 & $2.9150 \%$ & $5.7295 \%$ & $4.4685 \%$ & $2.4927 \%$ & $5.4641 \%$ & $6.3350 \%$ & $6.6424 \%$ & $6.6361 \%$ & $3.2957 \%$ \\
18 & $3.5242 \%$ & $6.3926 \%$ & $4.5046 \%$ & $2.4698 \%$ & $5.7973 \%$ & $6.2832 \%$ & $6.3449 \%$ & $6.3185 \%$ & $5.3781 \%$ \\
\hline Average & $1.8420 \%$ & $3.5514 \%$ & $3.1219 \%$ & $2.2936 \%$ & $3.4150 \%$ & $3.7206 \%$ & $3.6650 \%$ & $3.8346 \%$ & $1.7854 \%$ \\
\hline
\end{tabular}

Appendix Table 6 This table shows the accuracy of monthly forecasts of US tourist arrivals from Korea. The Absolute Percentage Error (APE) is calculated at forecasting horizon 1 to 18 months (1.5 years). The ARFIMA, ARIMA, and SARIMA models are all estimated by the data from Jan 1996 to Jun 2013 (210 months).

\begin{tabular}{rrrr}
\hline horizon & ARFIMA & \multicolumn{1}{l}{ ARIMA } & SARIMA \\
\hline 1 & $1.0212 \%$ & $40.2943 \%$ & $4.9225 \%$ \\
2 & $1.1793 \%$ & $45.6519 \%$ & $5.1590 \%$ \\
3 & $2.7185 \%$ & $48.2972 \%$ & $6.1137 \%$ \\
4 & $3.6141 \%$ & $49.8343 \%$ & $6.2708 \%$ \\
5 & $7.3728 \%$ & $59.0620 \%$ & $7.4147 \%$ \\
6 & $9.1359 \%$ & $59.9481 \%$ & $7.4155 \%$ \\
7 & $9.6288 \%$ & $60.0256 \%$ & $8.5526 \%$ \\
8 & $10.2235 \%$ & $60.9766 \%$ & $8.2167 \%$ \\
9 & $10.3071 \%$ & $72.7159 \%$ & $9.3587 \%$ \\
10 & $10.6437 \%$ & $75.5424 \%$ & $9.9909 \%$ \\
11 & $10.7144 \%$ & $76.4881 \%$ & $10.1821 \%$ \\
12 & $11.2069 \%$ & $82.4094 \%$ & $11.0282 \%$ \\
13 & $11.3557 \%$ & $95.9604 \%$ & $13.5403 \%$ \\
14 & $15.5508 \%$ & $99.8409 \%$ & $14.5765 \%$ \\
15 & $16.5127 \%$ & $101.0149 \%$ & $14.7895 \%$ \\
16 & $17.0306 \%$ & $102.7707 \%$ & $15.0306 \%$ \\
17 & $17.2215 \%$ & $103.1700 \%$ & $14.9428 \%$ \\
18 & $18.9664 \%$ & $106.2999 \%$ & $15.6618 \%$ \\
\hline Average & $10.2447 \%$ & $74.4613 \%$ & $10.1759 \%$ \\
\hline & & &
\end{tabular}

Appendix Table 7 This table shows the accuracy of monthly forecasts of US tourist arrivals from Brazil. The Absolute Percentage Error (APE) is calculated at forecasting horizon 1 to 18 months (1.5 years). The $p$ NN-HP, $p$ NN-WT and $p$ NNMA models are all estimated by the data from Jan 1996 to Jun 2013 (210 months).

\begin{tabular}{rrrrr|rrrr|r}
\hline \multicolumn{5}{c|}{$p$} & \multicolumn{4}{c|}{$p$ NN WT } & \multirow{2}{*}{$p$ NN MA } \\
\cline { 1 - 7 } horizon & 100 & 1600 & 3600 & 129600 & 3 & 4 & 5 & 6 & \\
\hline 1 & $1.3148 \%$ & $1.3157 \%$ & $1.2996 \%$ & $1.6014 \%$ & $1.1027 \%$ & $1.6325 \%$ & $0.4105 \%$ & $1.5342 \%$ & $6.4911 \%$ \\
2 & $1.1854 \%$ & $1.4568 \%$ & $1.2024 \%$ & $1.7146 \%$ & $2.3684 \%$ & $1.7444 \%$ & $0.9321 \%$ & $0.6227 \%$ & $6.2555 \%$ \\
3 & $1.5759 \%$ & $1.5058 \%$ & $0.9284 \%$ & $2.2031 \%$ & $3.1255 \%$ & $2.0195 \%$ & $0.5687 \%$ & $2.1947 \%$ & $7.1236 \%$ \\
4 & $1.3133 \%$ & $1.8015 \%$ & $0.6091 \%$ & $1.5763 \%$ & $3.1031 \%$ & $1.8940 \%$ & $0.3857 \%$ & $2.4193 \%$ & $7.8627 \%$ \\
5 & $1.7709 \%$ & $1.1799 \%$ & $0.1296 \%$ & $2.0603 \%$ & $2.9586 \%$ & $2.1028 \%$ & $0.6385 \%$ & $1.3198 \%$ & $5.8389 \%$ \\
6 & $1.7642 \%$ & $1.2354 \%$ & $0.1145 \%$ & $2.4207 \%$ & $3.6302 \%$ & $2.3235 \%$ & $1.2144 \%$ & $2.9056 \%$ & $8.2058 \%$ \\
7 & $1.8849 \%$ & $1.7822 \%$ & $0.4559 \%$ & $2.6932 \%$ & $3.0910 \%$ & $2.6384 \%$ & $1.4932 \%$ & $2.5594 \%$ & $6.6710 \%$ \\
8 & $1.8398 \%$ & $1.3467 \%$ & $2.3833 \%$ & $2.6229 \%$ & $4.5511 \%$ & $2.5843 \%$ & $1.6140 \%$ & $1.7579 \%$ & $7.9699 \%$ \\
9 & $1.1596 \%$ & $1.4412 \%$ & $0.8936 \%$ & $2.5452 \%$ & $4.9128 \%$ & $2.6003 \%$ & $1.3346 \%$ & $2.9001 \%$ & $7.5681 \%$ \\
10 & $1.0097 \%$ & $1.1508 \%$ & $0.6286 \%$ & $2.8015 \%$ & $2.6906 \%$ & $2.7733 \%$ & $1.7148 \%$ & $3.5923 \%$ & $8.6463 \%$ \\
11 & $1.2467 \%$ & $1.9779 \%$ & $1.2006 \%$ & $2.8034 \%$ & $1.9696 \%$ & $2.7593 \%$ & $2.6644 \%$ & $3.5457 \%$ & $5.9343 \%$
\end{tabular}




\begin{tabular}{rrrrr|rrrr|r}
12 & $1.9950 \%$ & $1.2839 \%$ & $2.5097 \%$ & $1.8182 \%$ & $2.3548 \%$ & $2.0013 \%$ & $1.8294 \%$ & $2.6868 \%$ & $7.0344 \%$ \\
13 & $1.9632 \%$ & $3.8194 \%$ & $3.3020 \%$ & $4.9486 \%$ & $3.0081 \%$ & $4.9490 \%$ & $3.7304 \%$ & $5.6887 \%$ & $8.1576 \%$ \\
14 & $1.6685 \%$ & $2.6262 \%$ & $2.5150 \%$ & $4.6405 \%$ & $4.5543 \%$ & $4.3311 \%$ & $4.8336 \%$ & $6.0538 \%$ & $10.2366 \%$ \\
15 & $1.4854 \%$ & $2.4558 \%$ & $2.1180 \%$ & $4.4165 \%$ & $4.5722 \%$ & $3.9915 \%$ & $4.6007 \%$ & $6.0185 \%$ & $11.2818 \%$ \\
16 & $2.0924 \%$ & $2.1158 \%$ & $1.7462 \%$ & $3.9444 \%$ & $4.7134 \%$ & $5.1480 \%$ & $4.6074 \%$ & $5.4903 \%$ & $10.2558 \%$ \\
17 & $2.2263 \%$ & $2.1411 \%$ & $1.8476 \%$ & $3.9080 \%$ & $4.1987 \%$ & $4.9432 \%$ & $4.4827 \%$ & $5.8068 \%$ & $11.3818 \%$ \\
18 & $2.3177 \%$ & $2.1855 \%$ & $1.4543 \%$ & $4.4550 \%$ & $5.1995 \%$ & $5.4667 \%$ & $5.1222 \%$ & $6.7383 \%$ & $11.6358 \%$ \\
\hline Average & $1.6563 \%$ & $1.8234 \%$ & $1.4077 \%$ & $2.9541 \%$ & $3.4503 \%$ & $3.1057 \%$ & $2.3432 \%$ & $3.5464 \%$ & $8.2528 \%$ \\
\hline
\end{tabular}

Appendix Table 8 This table shows the accuracy of monthly forecasts of US tourist arrivals from Brazil. The Absolute Percentage Error (APE) is calculated at forecasting horizon 1 to 18 months (1.5 years). The ARFIMA, ARIMA, and SARIMA models are all estimated by the data from Jan 1996 to Jun 2013 (210 months).

\begin{tabular}{rrrr}
\hline horizon & ARFIMA & ARIMA & SARIMA \\
\hline 1 & $1.2278 \%$ & $1.8161 \%$ & $1.7951 \%$ \\
2 & $1.6294 \%$ & $2.5810 \%$ & $1.9721 \%$ \\
3 & $1.7329 \%$ & $3.6132 \%$ & $2.4174 \%$ \\
4 & $4.3379 \%$ & $6.7773 \%$ & $2.9164 \%$ \\
5 & $5.0071 \%$ & $10.1477 \%$ & $3.0140 \%$ \\
6 & $6.0240 \%$ & $12.0452 \%$ & $3.8076 \%$ \\
7 & $6.2887 \%$ & $13.8791 \%$ & $3.9488 \%$ \\
8 & $6.4496 \%$ & $14.2311 \%$ & $4.3046 \%$ \\
9 & $7.3459 \%$ & $15.3354 \%$ & $4.3670 \%$ \\
10 & $8.4452 \%$ & $15.5091 \%$ & $4.4511 \%$ \\
11 & $9.7125 \%$ & $15.8001 \%$ & $4.5104 \%$ \\
12 & $11.2433 \%$ & $16.0689 \%$ & $4.6205 \%$ \\
13 & $13.5170 \%$ & $16.2644 \%$ & $6.3044 \%$ \\
14 & $14.2496 \%$ & $16.4207 \%$ & $6.5573 \%$ \\
15 & $16.0443 \%$ & $23.3100 \%$ & $7.2995 \%$ \\
16 & $17.1511 \%$ & $26.7056 \%$ & $7.6337 \%$ \\
17 & $24.2760 \%$ & $29.8989 \%$ & $8.6465 \%$ \\
18 & $25.7695 \%$ & $32.4431 \%$ & $9.3443 \%$ \\
\hline Average & $10.0251 \%$ & $15.1582 \%$ & $4.8839 \%$ \\
\hline & & &
\end{tabular}

Appendix Table 9 This table shows the accuracy of monthly forecasts of US tourist arrivals from Germany. The Absolute Percentage Error (APE) is calculated at forecasting horizon 1 to 18 months (1.5 years). The $p$ NN-HP, $p$ NN-WT and $p$ NNMA models are all estimated by the data from Jan 1996 to Jun 2013 (210 months).

\begin{tabular}{rrrrr|rrrr|r}
\hline \multicolumn{7}{c|}{$p$ NN HP } & \multicolumn{4}{c|}{$p$ NN WT } & \multirow{2}{*}{$p$ NN MA } \\
\cline { 1 - 7 } horizon & 100 & 1600 & 3600 & 129600 & 3 & 4 & 5 & 6 & \\
\hline 1 & $1.3747 \%$ & $1.3963 \%$ & $1.1930 \%$ & $2.4732 \%$ & $1.7424 \%$ & $3.3753 \%$ & $1.5811 \%$ & $2.9906 \%$ & $1.1292 \%$ \\
2 & $0.2788 \%$ & $1.1002 \%$ & $1.8228 \%$ & $1.5029 \%$ & $2.4173 \%$ & $0.6718 \%$ & $0.0774 \%$ & $0.2353 \%$ & $4.5481 \%$ \\
3 & $2.0942 \%$ & $2.0028 \%$ & $1.9568 \%$ & $1.2903 \%$ & $1.9508 \%$ & $1.8382 \%$ & $1.8995 \%$ & $1.8291 \%$ & $4.3346 \%$ \\
4 & $2.0688 \%$ & $1.9966 \%$ & $1.9746 \%$ & $2.0009 \%$ & $1.9539 \%$ & $1.8514 \%$ & $1.9853 \%$ & $2.0091 \%$ & $1.6044 \%$ \\
5 & $1.5305 \%$ & $1.5750 \%$ & $1.3193 \%$ & $1.4305 \%$ & $2.3995 \%$ & $1.5504 \%$ & $1.6655 \%$ & $1.5738 \%$ & $1.5521 \%$ \\
6 & $2.2150 \%$ & $2.1550 \%$ & $2.1064 \%$ & $2.0691 \%$ & $1.9896 \%$ & $2.0011 \%$ & $2.0684 \%$ & $2.0218 \%$ & $1.7604 \%$ \\
7 & $2.8600 \%$ & $2.8856 \%$ & $2.2503 \%$ & $2.2092 \%$ & $2.7073 \%$ & $2.9193 \%$ & $2.2023 \%$ & $2.9231 \%$ & $4.1862 \%$
\end{tabular}




\begin{tabular}{rrrrr|rrrr|r}
8 & $2.2194 \%$ & $3.7543 \%$ & $1.8798 \%$ & $3.4148 \%$ & $3.7400 \%$ & $1.8699 \%$ & $3.4674 \%$ & $3.4452 \%$ & $4.7275 \%$ \\
9 & $2.6896 \%$ & $2.6714 \%$ & $3.6748 \%$ & $2.7512 \%$ & $2.1952 \%$ & $2.8959 \%$ & $2.2332 \%$ & $2.2801 \%$ & $2.1742 \%$ \\
10 & $4.5425 \%$ & $4.3224 \%$ & $4.6863 \%$ & $3.4350 \%$ & $4.4437 \%$ & $4.4285 \%$ & $4.3965 \%$ & $4.3622 \%$ & $7.9458 \%$ \\
11 & $2.2572 \%$ & $2.9785 \%$ & $2.8687 \%$ & $2.6026 \%$ & $4.8680 \%$ & $4.3558 \%$ & $5.0410 \%$ & $5.0801 \%$ & $2.6359 \%$ \\
12 & $2.7766 \%$ & $2.8904 \%$ & $3.2677 \%$ & $3.1058 \%$ & $3.5626 \%$ & $3.3756 \%$ & $2.9172 \%$ & $3.1653 \%$ & $7.4599 \%$ \\
13 & $1.3716 \%$ & $8.1055 \%$ & $1.3527 \%$ & $8.0117 \%$ & $5.8010 \%$ & $7.4439 \%$ & $2.9191 \%$ & $9.0719 \%$ & $8.6162 \%$ \\
14 & $3.5438 \%$ & $3.6720 \%$ & $7.7640 \%$ & $6.3288 \%$ & $7.3803 \%$ & $7.9645 \%$ & $4.4215 \%$ & $8.2972 \%$ & $10.3059 \%$ \\
15 & $5.5490 \%$ & $5.6997 \%$ & $6.6720 \%$ & $6.6839 \%$ & $4.6340 \%$ & $6.6862 \%$ & $5.7135 \%$ & $6.4690 \%$ & $7.8736 \%$ \\
16 & $6.2010 \%$ & $6.4751 \%$ & $6.5948 \%$ & $6.5131 \%$ & $6.1507 \%$ & $6.0853 \%$ & $6.4898 \%$ & $6.5767 \%$ & $2.8960 \%$ \\
17 & $5.5697 \%$ & $6.0234 \%$ & $5.9989 \%$ & $5.9879 \%$ & $6.4946 \%$ & $5.9463 \%$ & $5.8716 \%$ & $7.3149 \%$ & $4.5734 \%$ \\
18 & $6.8429 \%$ & $7.0628 \%$ & $6.8377 \%$ & $6.7239 \%$ & $6.7716 \%$ & $6.8786 \%$ & $6.7940 \%$ & $6.6479 \%$ & $5.3509 \%$ \\
\hline Average & $3.1103 \%$ & $3.7093 \%$ & $3.5678 \%$ & $3.8075 \%$ & $3.9557 \%$ & $4.0077 \%$ & $3.4302 \%$ & $4.2385 \%$ & $4.6486 \%$ \\
\hline
\end{tabular}

Appendix Table 10 This table shows the accuracy of monthly forecasts of US tourist arrivals from Germany. The Absolute Percentage Error (APE) is calculated at forecasting horizon 1 to 18 months (1.5 years). The ARFIMA, ARIMA, and SARIMA models are all estimated by the data from Jan 1996 to Jun 2013 (210 months).

\begin{tabular}{rrrr}
\hline horizon & ARFIMA & ARIMA & SARIMA \\
\hline 1 & $0.3397 \%$ & $3.4469 \%$ & $1.9129 \%$ \\
2 & $3.4448 \%$ & $6.9276 \%$ & $2.0934 \%$ \\
3 & $4.8205 \%$ & $4.5628 \%$ & $2.5981 \%$ \\
4 & $5.0343 \%$ & $7.7673 \%$ & $2.7497 \%$ \\
5 & $5.3356 \%$ & $5.1066 \%$ & $2.2763 \%$ \\
6 & $6.4019 \%$ & $5.1428 \%$ & $2.7210 \%$ \\
7 & $7.1616 \%$ & $2.9461 \%$ & $3.2046 \%$ \\
8 & $9.6190 \%$ & $6.5605 \%$ & $4.0634 \%$ \\
9 & $12.9312 \%$ & $86.8930 \%$ & $11.2173 \%$ \\
10 & $13.3089 \%$ & $8.9147 \%$ & $5.8897 \%$ \\
11 & $14.1300 \%$ & $11.5817 \%$ & $5.3091 \%$ \\
12 & $15.5623 \%$ & $13.1765 \%$ & $5.5691 \%$ \\
13 & $16.4700 \%$ & $22.5850 \%$ & $8.3408 \%$ \\
14 & $17.0988 \%$ & $49.4202 \%$ & $11.4724 \%$ \\
15 & $17.2917 \%$ & $14.0700 \%$ & $7.9402 \%$ \\
16 & $25.2028 \%$ & $32.0970 \%$ & $10.1166 \%$ \\
17 & $43.4020 \%$ & $49.6664 \%$ & $13.3499 \%$ \\
18 & $61.3051 \%$ & $69.1981 \%$ & $17.3103 \%$ \\
\hline Average & $15.4922 \%$ & $22.2257 \%$ & $6.5630 \%$ \\
\hline & & &
\end{tabular}

Appendix Table 11 This table shows the accuracy of monthly forecasts of US tourist arrivals from Australia. The Absolute Percentage Error (APE) is calculated at forecasting horizon 1 to 18 months (1.5 years). The $p \mathrm{NN}-\mathrm{HP}, p \mathrm{NN}-\mathrm{WT}$ and $p \mathrm{NN}-$ MA models are all estimated by the data from Jan 1996 to Jun 2013 (210 months).

\begin{tabular}{rrrrr|rrrr|r}
\hline \multicolumn{5}{c|}{$p$ NN HP } & \multicolumn{4}{c|}{$p$ NN WT } & \multirow{2}{*}{$p$ NN MA } \\
\cline { 1 - 8 } horizon & 100 & 1600 & 3600 & 129600 & 3 & 4 & 5 & 6 & \\
\hline 1 & $1.9902 \%$ & $1.7584 \%$ & $1.3113 \%$ & $1.8793 \%$ & $1.8799 \%$ & $1.5088 \%$ & $1.3197 \%$ & $1.3533 \%$ & $4.2470 \%$ \\
2 & $0.7915 \%$ & $3.4844 \%$ & $3.3466 \%$ & $1.2105 \%$ & $2.2083 \%$ & $1.0747 \%$ & $3.2869 \%$ & $1.8116 \%$ & $9.0320 \%$ \\
3 & $2.2215 \%$ & $1.8084 \%$ & $1.3281 \%$ & $1.9282 \%$ & $1.6308 \%$ & $2.0081 \%$ & $1.8743 \%$ & $1.9081 \%$ & $0.0180 \%$
\end{tabular}




\begin{tabular}{rrrrr|rrrr|r}
4 & $2.0622 \%$ & $1.4811 \%$ & $1.2220 \%$ & $1.9650 \%$ & $2.0829 \%$ & $2.9136 \%$ & $1.9640 \%$ & $1.5042 \%$ & $3.1279 \%$ \\
5 & $2.2829 \%$ & $1.5167 \%$ & $1.4188 \%$ & $2.1496 \%$ & $2.2995 \%$ & $2.0281 \%$ & $2.2308 \%$ & $2.6138 \%$ & $3.0510 \%$ \\
6 & $1.2340 \%$ & $1.7473 \%$ & $1.3688 \%$ & $1.2164 \%$ & $2.5208 \%$ & $2.3013 \%$ & $1.2925 \%$ & $2.9022 \%$ & $6.3291 \%$ \\
7 & $1.7498 \%$ & $1.3359 \%$ & $1.1883 \%$ & $2.4317 \%$ & $1.6341 \%$ & $2.0183 \%$ & $2.2896 \%$ & $2.4823 \%$ & $1.4221 \%$ \\
8 & $1.5632 \%$ & $1.9854 \%$ & $1.7670 \%$ & $2.5236 \%$ & $2.1161 \%$ & $2.0258 \%$ & $2.0042 \%$ & $2.6041 \%$ & $4.7428 \%$ \\
9 & $0.7428 \%$ & $1.7436 \%$ & $2.6982 \%$ & $1.9275 \%$ & $2.5382 \%$ & $3.8160 \%$ & $2.9935 \%$ & $2.6431 \%$ & $5.9798 \%$ \\
10 & $1.8219 \%$ & $1.8781 \%$ & $2.5905 \%$ & $3.2995 \%$ & $3.5053 \%$ & $3.3006 \%$ & $3.2688 \%$ & $4.1393 \%$ & $8.7299 \%$ \\
11 & $0.7649 \%$ & $2.5537 \%$ & $2.3230 \%$ & $2.5874 \%$ & $5.1502 \%$ & $4.8710 \%$ & $3.2945 \%$ & $4.5305 \%$ & $12.3760 \%$ \\
12 & $2.1226 \%$ & $2.0248 \%$ & $2.7298 \%$ & $3.8244 \%$ & $5.0801 \%$ & $5.6411 \%$ & $4.3353 \%$ & $4.3889 \%$ & $8.6401 \%$ \\
13 & $1.0951 \%$ & $2.8322 \%$ & $2.0142 \%$ & $3.1739 \%$ & $5.6447 \%$ & $5.2472 \%$ & $6.3051 \%$ & $4.5756 \%$ & $10.4457 \%$ \\
14 & $1.1351 \%$ & $3.1263 \%$ & $2.9730 \%$ & $1.4372 \%$ & $5.4761 \%$ & $6.8146 \%$ & $6.6748 \%$ & $6.8685 \%$ & $16.9133 \%$ \\
15 & $3.2942 \%$ & $2.5174 \%$ & $2.0307 \%$ & $5.0133 \%$ & $5.7394 \%$ & $6.7840 \%$ & $6.9017 \%$ & $6.8481 \%$ & $7.0926 \%$ \\
16 & $3.0780 \%$ & $2.3211 \%$ & $2.8475 \%$ & $4.7522 \%$ & $5.4360 \%$ & $6.1290 \%$ & $6.4867 \%$ & $6.6519 \%$ & $10.8718 \%$ \\
17 & $3.8895 \%$ & $2.2135 \%$ & $3.0543 \%$ & $4.5770 \%$ & $5.6120 \%$ & $6.7304 \%$ & $6.2120 \%$ & $6.1186 \%$ & $13.2559 \%$ \\
18 & $2.7448 \%$ & $2.4744 \%$ & $3.1659 \%$ & $4.7273 \%$ & $5.6026 \%$ & $6.0958 \%$ & $7.2296 \%$ & $7.5436 \%$ & $13.1924 \%$ \\
\hline Average & $1.9213 \%$ & $2.1557 \%$ & $2.1877 \%$ & $2.8124 \%$ & $3.6754 \%$ & $3.9616 \%$ & $3.8869 \%$ & $3.9715 \%$ & $7.7482 \%$ \\
\hline
\end{tabular}

Appendix Table 12 This table shows the accuracy of monthly forecasts of US tourist arrivals from Australia. The Absolute Percentage Error (APE) is calculated at forecasting horizon 1 to 18 months (1.5 years). The ARFIMA, ARIMA, and SARIMA models are all estimated by the data from Jan 1996 to Jun 2013 (210 months).

\begin{tabular}{rrrr}
\hline horizon & ARFIMA & ARIMA & SARIMA \\
\hline 1 & $0.1630 \%$ & $0.1627 \%$ & $1.5976 \%$ \\
2 & $1.5407 \%$ & $3.8420 \%$ & $2.8754 \%$ \\
3 & $2.4711 \%$ & $3.9942 \%$ & $1.9264 \%$ \\
4 & $2.8186 \%$ & $4.4232 \%$ & $2.3241 \%$ \\
5 & $3.1204 \%$ & $7.5974 \%$ & $2.7554 \%$ \\
6 & $6.1417 \%$ & $8.0092 \%$ & $3.1876 \%$ \\
7 & $7.7945 \%$ & $9.5544 \%$ & $3.0819 \%$ \\
8 & $10.1134 \%$ & $9.9570 \%$ & $3.7639 \%$ \\
9 & $10.1771 \%$ & $15.1617 \%$ & $4.5838 \%$ \\
10 & $10.7876 \%$ & $15.5402 \%$ & $5.3511 \%$ \\
11 & $13.1222 \%$ & $19.3484 \%$ & $6.4474 \%$ \\
12 & $18.6795 \%$ & $19.6015 \%$ & $7.0062 \%$ \\
13 & $19.3294 \%$ & $21.9494 \%$ & $7.5102 \%$ \\
14 & $20.3376 \%$ & $29.0222 \%$ & $9.1617 \%$ \\
15 & $21.2042 \%$ & $34.4473 \%$ & $9.2612 \%$ \\
16 & $21.5191 \%$ & $41.0450 \%$ & $10.1035 \%$ \\
17 & $23.2741 \%$ & $49.6832 \%$ & $11.3291 \%$ \\
18 & $30.5076 \%$ & $78.1964 \%$ & $14.6800 \%$ \\
\hline Average & $12.3945 \%$ & $20.6409 \%$ & $5.9415 \%$ \\
\hline & & &
\end{tabular}


Appendix Table 13 This table shows the average accuracy of monthly forecasts of US tourist arrivals from ITALY. The MAPE and RMSE are average values across all forecasted data from Aug 2013 to Sep 2017. The models are all estimated by the data from Jan 1996 to Jun 2013.

\begin{tabular}{|c|c|c|c|c|c|c|}
\hline ITALY & \multicolumn{3}{|c|}{ MAPE (\%) } & \multicolumn{3}{|c|}{ RMSE } \\
\hline Horizon & pNN-HP & pNN-MA & $\begin{array}{c}\text { SARIM } \\
\text { A }\end{array}$ & pNN-HP & pNN-MA & SARIMA \\
\hline 1 & 2.0760 & 2.2960 & 5.8267 & $1,475.6624$ & $1,457.7917$ & 125.6543 \\
\hline 2 & 2.1643 & 2.2975 & 6.5535 & $1,666.8365$ & $1,550.6151$ & 616.0718 \\
\hline 3 & 2.4231 & 2.4264 & 6.9084 & $1,771.6161$ & $1,586.2061$ & $1,174.8861$ \\
\hline 4 & 2.5114 & 2.4301 & 7.7232 & $1,788.0513$ & $1,635.6921$ & $1,445.5568$ \\
\hline 5 & 2.5947 & 2.5749 & 7.8176 & $1,813.4666$ & $1,995.6738$ & $1,568.5179$ \\
\hline 6 & 2.8404 & 2.5902 & 7.9839 & $1,917.3266$ & $2,094.8811$ & $1,692.6787$ \\
\hline 7 & 2.8780 & 2.7442 & 8.6795 & $2,044.4124$ & $2,466.9727$ & $2,832.3098$ \\
\hline 8 & 2.9147 & 3.3665 & 8.8374 & $2,054.7212$ & $2,547.7043$ & $2,967.9491$ \\
\hline 9 & 2.9329 & 3.5887 & 8.9095 & $2,074.7729$ & $2,750.9733$ & $3,061.8112$ \\
\hline 10 & 3.0640 & 3.6322 & 9.0293 & $2,134.1112$ & $2,859.2173$ & $3,497.8551$ \\
\hline 11 & 3.1256 & 3.7910 & 9.2291 & $2,418.3283$ & $3,421.4189$ & $4,358.8237$ \\
\hline 12 & 3.1793 & 4.1297 & 9.7662 & $2,430.2504$ & $3,676.2009$ & $6,895.9762$ \\
\hline 13 & 3.2600 & 4.3187 & 10.0474 & $2,433.6272$ & $3,807.4520$ & $7,259.1092$ \\
\hline 14 & 3.2896 & 4.4406 & 10.3074 & $2,797.2310$ & $3,862.3840$ & $7,367.2322$ \\
\hline 15 & 4.0717 & 4.8030 & 10.5094 & $2,917.3847$ & $3,900.5534$ & $7,693.2765$ \\
\hline 16 & 4.1003 & 4.9981 & 11.2053 & $3,357.8388$ & $4,175.0139$ & $8,148.8646$ \\
\hline 17 & 4.4225 & 5.6311 & 11.7831 & $3,897.4073$ & $4,180.4292$ & $8,360.5889$ \\
\hline 18 & 5.1484 & 5.9064 & 11.8083 & $4,032.1618$ & $4,204.3810$ & $9,958.6574$ \\
\hline
\end{tabular}

Note: MAPE is Mean Average Absolute Percentage Error; RMSE is Root Mean Square Error; pNN-HP is the paired Neural Network model with HP filter; pNN-MA is the paired Neural Network model with Moving Average as the trend filter; SARIMA is the Seasonal Autoregressive Integrated Moving Average.

Appendix Table 14 This table shows the average accuracy of monthly forecasts of US tourist arrivals from SPAIN. The MAPE and RMSE are average values across all forecasted data from Aug 2013 to Sep 2017. The models are all estimated by the data from Jan 1996 to Jul 2013.

\begin{tabular}{|c|c|c|c|c|c|c|}
\hline SPAIN & \multicolumn{3}{|c|}{ MAPE (\%) } & \multicolumn{3}{|c|}{ RMSE } \\
\hline Horizon & pNN-HP & pNN-MA & $\begin{array}{c}\text { SARIM } \\
\text { A }\end{array}$ & pNN-HP & pNN-MA & SARIMA \\
\hline 1 & 2.0620 & 2.0070 & 7.5984 & $1,131.2550$ & 967.7461 & 433.4365 \\
\hline 2 & 2.2655 & 2.0861 & 7.8965 & $1,159.9743$ & 969.5743 & 854.8061 \\
\hline 3 & 2.2796 & 2.1114 & 8.4786 & $1,167.8067$ & $1,299.0070$ & $1,055.4889$ \\
\hline 4 & 2.4873 & 2.1931 & 8.5875 & $1,306.2908$ & $1,334.5188$ & $1,082.1928$ \\
\hline 5 & 2.5127 & 2.2924 & 9.2462 & $1,338.3505$ & $1,396.7860$ & $1,330.7147$ \\
\hline 6 & 2.5820 & 2.3603 & 9.8384 & $1,371.1106$ & $1,538.6468$ & $2,073.4365$ \\
\hline 7 & 2.6814 & 2.6981 & 10.1559 & $1,403.6249$ & $1,550.6383$ & $2,444.0618$ \\
\hline 8 & 2.8738 & 2.7678 & 10.1704 & $1,469.7818$ & $1,553.7871$ & $2,669.5379$ \\
\hline 9 & 3.1616 & 2.7878 & 10.1807 & $1,629.6006$ & $1,649.0506$ & $2,816.0434$ \\
\hline 10 & 3.3143 & 3.0014 & 10.4074 & $1,682.1278$ & $1,698.8751$ & $3,545.6167$ \\
\hline 11 & 3.3900 & 3.8057 & 10.8182 & $1,744.6072$ & $1,761.1165$ & $3,605.0670$ \\
\hline 12 & 3.4045 & 3.8778 & 10.9827 & $2,063.5352$ & $1,808.4086$ & $4,142.6767$ \\
\hline 13 & 3.7876 & 3.8861 & 11.2368 & $2,419.1042$ & $1,893.1316$ & $4,225.0093$ \\
\hline 14 & 3.9407 & 3.8905 & 11.3642 & $2,725.9535$ & $2,011.4587$ & $4,245.9654$ \\
\hline 15 & 5.1572 & 4.0116 & 11.3889 & $2,913.4500$ & $2,376.6354$ & $5,092.6010$ \\
\hline 16 & 5.7490 & 4.1130 & 11.6754 & $3,181.5540$ & $2,568.4638$ & $5,363.5469$ \\
\hline 17 & 5.7636 & 4.1994 & 11.6985 & $3,286.3516$ & $2,740.7787$ & $5,946.4634$ \\
\hline 18 & 6.3312 & 4.5619 & 12.1358 & $3,287.6573$ & $2,776.1523$ & $6,443.4930$ \\
\hline
\end{tabular}

Note: MAPE is Mean Average Absolute Percentage Error; RMSE is Root Mean Square Error; pNN-HP is the paired Neural Network model with HP filter; pNN-MA is the paired Neural Network model with Moving Average as the trend filter; SARIMA is the Seasonal Autoregressive Integrated Moving Average. 
Appendix Table 15 This table shows the average accuracy of monthly forecasts of US tourist arrivals from KOREA. The MAPE and RMSE are average values across all forecasted data from Aug 2013 to Sep 2017. The models are all estimated by the data from Jan 1996 to Jul 2013.

\begin{tabular}{|c|c|c|c|c|c|c|}
\hline KOREA & \multicolumn{3}{|c|}{ MAPE (\%) } & \multicolumn{3}{|c|}{ RMSE } \\
\hline Horizon & pNN-HP & pNN-MA & $\begin{array}{c}\text { SARIM } \\
\text { A }\end{array}$ & pNN-HP & pNN-MA & SARIMA \\
\hline 1 & 2.0793 & 2.1199 & 6.3157 & $2,640.2352$ & $3,046.9965$ & $1,076.3064$ \\
\hline 2 & 2.1052 & 3.8615 & 7.8786 & $2,952.5378$ & $4,879.4001$ & $3,555.9646$ \\
\hline 3 & 2.1470 & 4.1527 & 9.9998 & $3,085.8545$ & $4,984.1086$ & $3,593.8022$ \\
\hline 4 & 2.2451 & 4.2305 & 10.2241 & $3,086.3512$ & $5,002.5364$ & $4,073.1923$ \\
\hline 5 & 2.4745 & 4.2319 & 10.6832 & $3,471.6760$ & $5,128.7030$ & $6,417.4305$ \\
\hline 6 & 3.0370 & 4.6459 & 10.8793 & $3,804.4224$ & $5,592.8176$ & $6,577.5964$ \\
\hline 7 & 3.6132 & 4.7867 & 10.8827 & $4,032.4997$ & $6,136.6385$ & $7,526.4953$ \\
\hline 8 & 3.7898 & 5.2997 & 12.2962 & $4,650.4786$ & $6,279.4104$ & $10,987.4539$ \\
\hline 9 & 3.8106 & 7.5102 & 13.1233 & $5,191.7739$ & $9,398.1144$ & $13,322.1902$ \\
\hline 10 & 3.8585 & 7.5286 & 13.2552 & $5,511.4165$ & $9,484.0720$ & $13,774.7276$ \\
\hline 11 & 3.8876 & 8.4528 & 14.4313 & $5,819.9610$ & $10,094.5358$ & $21,796.1997$ \\
\hline 12 & 3.9205 & 9.0589 & 14.9495 & $5,879.5808$ & $10,315.7880$ & $25,144.5286$ \\
\hline 13 & 3.9252 & 9.1615 & 15.7532 & $6,364.2125$ & $13,809.4776$ & $25,911.6304$ \\
\hline 14 & 4.0039 & 9.3973 & 15.9212 & $6,407.3150$ & $14,805.1213$ & $26,315.7580$ \\
\hline 15 & 4.3950 & 9.9553 & 16.5303 & $6,593.0796$ & $16,049.2606$ & $28,834.7197$ \\
\hline 16 & 4.6872 & 10.4615 & 16.7469 & $6,869.8729$ & $16,449.2499$ & $32,216.6823$ \\
\hline 17 & 5.0658 & 10.6986 & 17.9139 & $7,006.9979$ & $16,760.7544$ & $36,164.1563$ \\
\hline 18 & 5.6340 & 10.7651 & 18.4079 & $8,701.1004$ & $17,084.5424$ & $36,280.4084$ \\
\hline
\end{tabular}

Note: MAPE is Mean Average Absolute Percentage Error; RMSE is Root Mean Square Error; pNN-HP is the paired Neural Network model with HP filter; pNN-MA is the paired Neural Network model with Moving Average as the trend filter; SARIMA is the Seasonal Autoregressive Integrated Moving Average.

Appendix Table 16 This table shows the average accuracy of monthly forecasts of US tourist arrivals from BRAZIL. The MAPE and RMSE are average values across all forecasted data from Aug 2013 to Sep 2017. The models are all estimated by the data from Jan 1996 to Jul 2013.

\begin{tabular}{|c|c|c|c|c|c|c|}
\hline BRAZIL & \multicolumn{3}{|c|}{ MAPE (\%) } & \multicolumn{3}{|c|}{ RMSE } \\
\hline Horizon & pNN-HP & pNN-MA & $\begin{array}{c}\text { SARIM } \\
\text { A }\end{array}$ & pNN-HP & pNN-MA & SARIMA \\
\hline 1 & 2.2335 & 2.5098 & 6.4360 & $3,057.5383$ & $4,257.6484$ & $2,045.6188$ \\
\hline 2 & 2.6828 & 3.1460 & 7.2434 & $3,392.3893$ & $5,259.9389$ & $7,878.5647$ \\
\hline 3 & 2.7026 & 3.1878 & 8.0854 & $3,521.7652$ & $5,283.6159$ & $8,763.6322$ \\
\hline 4 & 2.8603 & 3.4739 & 8.1748 & $4,420.9312$ & $5,399.7015$ & $13,016.4555$ \\
\hline 5 & 2.8874 & 3.6534 & 8.8473 & $4,506.7629$ & $5,406.3148$ & $15,660.7052$ \\
\hline 6 & 2.9132 & 3.7979 & 9.6939 & $4,537.9710$ & $5,574.7851$ & $15,943.6963$ \\
\hline 7 & 2.9218 & 3.8081 & 9.9693 & $4,545.9499$ & $5,634.5468$ & $16,920.2905$ \\
\hline 8 & 2.9962 & 3.8916 & 10.1870 & $4,863.4487$ & $5,654.2005$ & $20,694.9382$ \\
\hline 9 & 3.0359 & 4.1216 & 10.2380 & $4,935.2503$ & $6,305.7624$ & $22,745.5311$ \\
\hline 10 & 3.0871 & 4.5922 & 11.1329 & $5,222.5648$ & $6,567.7108$ & $22,793.9771$ \\
\hline 11 & 3.1031 & 4.6884 & 11.3045 & $6,409.7712$ & $6,625.3523$ & $25,452.6020$ \\
\hline 12 & 3.2811 & 4.7335 & 11.9521 & $6,524.7034$ & $6,719.7297$ & $26,467.4362$ \\
\hline 13 & 3.3392 & 5.0993 & 12.8084 & $6,857.0126$ & $6,893.2053$ & $26,535.0114$ \\
\hline 14 & 4.6565 & 5.1042 & 13.3779 & $7,413.8218$ & $6,911.0253$ & $28,440.4143$ \\
\hline 15 & 5.0524 & 5.1769 & 13.7426 & $7,822.6411$ & $7,187.5633$ & $29,432.2708$ \\
\hline 16 & 5.3771 & 5.3796 & 13.7932 & $9,764.8979$ & $8,337.4461$ & $30,144.2447$ \\
\hline 17 & 6.0188 & 5.4892 & 13.8172 & $10,977.9553$ & $8,693.2951$ & $30,384.7618$ \\
\hline 18 & 8.5497 & 5.7705 & 14.2168 & $15,743.3030$ & $8,827.6983$ & $31,155.7587$ \\
\hline
\end{tabular}

Note: MAPE is Mean Average Absolute Percentage Error; RMSE is Root Mean Square Error; pNN-HP is the paired Neural Network model with HP filter; pNN-MA is the paired Neural Network model with Moving Average as the trend filter; SARIMA is the Seasonal Autoregressive Integrated Moving Average. 
Appendix Table 17 This table shows the average accuracy of monthly forecasts of US tourist arrivals from GERMANY. The MAPE and RMSE are average values across all forecasted data from Aug 2013 to Sep 2017. The models are all estimated by the data from Jan 1996 to Jul 2013.

\begin{tabular}{|c|c|c|c|c|c|c|}
\hline \multicolumn{7}{|c|}{ GERMAN } \\
\hline Horizon & pNN-HP & pNN-MA & $\begin{array}{c}\text { SARIM } \\
\text { A }\end{array}$ & pNN-HP & pNN-MA & SARIMA \\
\hline 1 & 2.0232 & 2.0899 & 6.0737 & $2,886.2685$ & $2,814.0082$ & 17.0417 \\
\hline 2 & 2.0819 & 2.0990 & 7.0777 & $3,355.7972$ & $3,240.0784$ & 481.5342 \\
\hline 3 & 2.0951 & 2.1730 & 7.1654 & $3,677.2995$ & $3,761.6868$ & $2,754.1544$ \\
\hline 4 & 2.0962 & 2.2608 & 8.3062 & $3,690.3158$ & $4,086.0665$ & $4,853.5575$ \\
\hline 5 & 2.2106 & 2.2732 & 9.0259 & $4,077.4593$ & $4,089.7474$ & $5,529.2361$ \\
\hline 6 & 2.2189 & 2.2953 & 9.3118 & $4,082.0890$ & $4,112.9655$ & $8,141.9160$ \\
\hline 7 & 2.3521 & 2.2956 & 9.8658 & $4,176.1337$ & $4,213.4900$ & $8,243.8132$ \\
\hline 8 & 2.3563 & 2.3728 & 10.0324 & $4,251.6155$ & $4,736.7540$ & $9,294.0308$ \\
\hline 9 & 2.3769 & 2.5377 & 10.2361 & $4,350.0382$ & $5,049.1387$ & $10,224.6293$ \\
\hline 10 & 2.3779 & 2.6588 & 10.2936 & $4,417.1725$ & $5,282.7154$ & $12,388.9522$ \\
\hline 11 & 2.4945 & 2.7320 & 10.5710 & $4,695.4755$ & $5,571.7511$ & $12,506.2165$ \\
\hline 12 & 2.6526 & 2.8772 & 10.9605 & $4,968.6968$ & $5,641.2513$ & $14,158.8806$ \\
\hline 13 & 2.9490 & 2.9504 & 11.5012 & $4,992.0286$ & $6,083.6366$ & $15,079.0906$ \\
\hline 14 & 3.0304 & 3.1776 & 11.7935 & $5,167.5828$ & $6,146.0525$ & $15,180.6853$ \\
\hline 15 & 3.1270 & 3.3762 & 11.9868 & $5,358.2740$ & $6,291.6163$ & $15,260.8974$ \\
\hline 16 & 3.2708 & 3.5396 & 11.9950 & $6,048.0195$ & $6,560.1257$ & $15,562.5985$ \\
\hline 17 & 3.6697 & 3.9830 & 12.4405 & $6,385.2877$ & $6,812.1865$ & $17,830.4787$ \\
\hline 18 & 3.7681 & 4.0417 & 12.8322 & $6,582.6340$ & $6,880.3405$ & $18,372.8446$ \\
\hline
\end{tabular}

Note: MAPE is Mean Average Absolute Percentage Error; RMSE is Root Mean Square Error; pNN-HP is the paired Neural Network model with HP filter; pNN-MA is the paired Neural Network model with Moving Average as the trend filter; SARIMA is the Seasonal Autoregressive Integrated Moving Average.

Appendix Table 18 This table shows the average accuracy of monthly forecasts of US tourist arrivals from AUSTRALIA. The MAPE and RMSE are average values across all forecasted data from Aug 2013 to Sep 2017. The models are all estimated by the data from Jan 1996 to Jul 2013.

\begin{tabular}{|c|c|c|c|c|c|c|}
\hline $\begin{array}{l}\text { AUSTRAL } \\
\text { IA }\end{array}$ & & $\mathrm{APE}(\%)$ & & & RMSE & \\
\hline Horizon & pNN-HP & pNN-MA & $\begin{array}{c}\text { SARIM } \\
\mathrm{A} \\
\end{array}$ & pNN-HP & pNN-MA & SARIMA \\
\hline 1 & 2.0344 & 2.0963 & 5.8563 & $1,879.6087$ & $2,203.7164$ & 115.8073 \\
\hline 2 & 2.0633 & 2.1058 & 6.5257 & $1,976.2163$ & $2,296.9482$ & 843.5682 \\
\hline 3 & 2.1108 & 2.1105 & 7.3650 & $2,335.8330$ & $2,487.9154$ & $1,094.8731$ \\
\hline 4 & 2.1143 & 2.3146 & 7.3873 & $2,403.0227$ & $2,708.4169$ & $1,429.3211$ \\
\hline 5 & 2.1586 & 2.3347 & 7.3945 & $2,430.1701$ & $3,024.3907$ & $1,710.1054$ \\
\hline 6 & 2.2065 & 2.3538 & 7.6419 & $2,655.8755$ & $3,136.8702$ & $1,911.4456$ \\
\hline 7 & 2.2764 & 2.4584 & 7.9794 & $2,796.2365$ & $3,148.3399$ & $2,134.2615$ \\
\hline 8 & 2.4465 & 2.7246 & 8.0472 & $2,823.3480$ & $3,432.0916$ & $2,150.8918$ \\
\hline 9 & 2.6196 & 2.7901 & 8.7539 & $3,073.6260$ & $3,888.0412$ & $3,084.2868$ \\
\hline 10 & 2.6956 & 3.7065 & 8.9697 & $3,134.7780$ & $4,331.6185$ & $3,436.5370$ \\
\hline 11 & 2.7121 & 3.8835 & 9.0382 & $3,500.6858$ & $4,414.2942$ & $3,587.8382$ \\
\hline 12 & 2.7825 & 5.0907 & 9.1288 & $3,564.7621$ & $5,335.7646$ & $3,841.6217$ \\
\hline 13 & 3.1559 & 5.4759 & 9.3118 & $3,854.4347$ & $5,855.3615$ & $4,474.2256$ \\
\hline 14 & 3.2456 & 5.5153 & 9.6002 & $3,870.5337$ & $6,026.6730$ & $4,694.3326$ \\
\hline 15 & 3.4178 & 5.7663 & 9.6531 & $3,953.1031$ & $6,466.3063$ & $5,541.1998$ \\
\hline 16 & 3.4516 & 6.1835 & 9.9969 & $3,970.5294$ & $6,522.8144$ & $5,627.8470$ \\
\hline 17 & 3.7459 & 6.7793 & 10.1529 & $4,335.7868$ & $7,241.5357$ & $5,801.2891$ \\
\hline 18 & 3.9772 & 6.7820 & 10.3305 & $4,429.4164$ & $7,442.9502$ & $6,843.4489$ \\
\hline
\end{tabular}

Note: MAPE is Mean Average Absolute Percentage Error; RMSE is Root Mean Square Error; pNN-HP is the paired Neural Network model with HP filter; pNN-MA is the paired Neural Network model with Moving Average as the trend filter; SARIMA is the Seasonal Autoregressive Integrated Moving Average. 


\section{References}

Alleyne, D. (2006). Can Seasonal Unit Root Testing Improve the Forecasting Accuracy of Tourist Arrivals. Tourism Economics, 12, pp. 45-64.

Barunik, J., Krehlik, T., \& Vacha, L. (2016). Modeling and forecasting exchange rate volatility in time-frequency domain. European Journal of Operational Research, 329-340.

Baxter, M., \& King, R. G. (1999). Measuring Business Cycles Approximate Band-Pass Filters for Economic Time Series. Review of Economics and Statistics, 575-593.

Burger, C., Dohnal, M., Kathrada, M., \& Law, R. (2001). A practitioner's guide to time series methods for tourism demand forecasting - a case study of Durban, South Africa. Tourism Management, 403-409.

Butler, R. (2001). Seasonality in Tourism: Issues and Implications. In T. Baum, \& S. Lundtorp, Seasonality in Tourism (pp. 5-21). Oxford, UK: Pergamon.

Chen, J. L., Li, G., Wu, D. C., \& Shen, S. (2017). Forecasting Seasonal Tourism Demand Using a Multiseries Structural Time Series Method . Journal of Travel Research, 1-12.

Claveria, O., \& Torra, S. (2014). Forecasting tourism demand to Catalonia: Neural networks vs. time series models. Economic Modelling, 36, 220-228.

Daubechies, I. (1992). Ten lectures on wavelets (Vol. 61). Siam.

Fuller, W. A. (1976). Introduction to Statistical Time Series. Wiley.

Ghysels, E., \& Osborn, D. R. (2001). The Econometric Analysis of Seasonal Time Series. Cambridge, UK: Cambridge University Press.

Hagan, M. T., \& Menhaj., M. B. (1994). Training feedforward networks with the Marquardt algorithm. IEEE transactions on Neural Networks, 989-993.

Harris, R., Stoja, E., \& Yilmaz, F. (2011). A cyclical model of exchange rate volatility. Journal of Banking and Finance, 35, 3055-3064.

Harvey, A., \& Todd, P. (1983). Forecasting economic time series with structural and Box-Jenkins Model: A Case Study. Journal of Business \& Economic Statistics, 299-307.

Hassani, H., Silva, E. S., Antonakakis, N., Filis, G., \& Gupta, R. (2017). Forecasting accuracy evaluation of tourist arrivals. Annals of Tourism Research, 63, 112-127.

Haven, E., Liu, X., \& Shen, L. (2012). De-noising option prices with the wavelet method. European Journal of Operational Research, 104-112. 
Hodrick, R. J., \& Prescott, E. C. (1997). Postwar US business cycles: an empirical investigation. Journal of Money, credit, and Banking, 1-16.

Kim, J. H. (1999). Forecasting Monthly Tourist Departures from Australia. Tourism Economics, 5(3), 277-91.

Kon, S. C., \& Turner, W. L. (2005). Neural Network Forecasting of Tourism Demand. Tourism Economics, 11, 301-28.

Kristjanpoller, W., \& Minutolo, M. (2016). Forecasting volatility of oil price using an artificial neural network-GARCH model. Expert Systems With Applications, 65, 233-241.

Kristjanpoller, W., Fadic, A., \& Minutolo, M. C. (2014). Volatility forecast using hybrid Neural Network models. Expert Systems with Applications, 2437-2442.

Kulendran, N., \& Witt, S. F. (2003). Forecasting the Demand for International Business Tourism. Journal of Travel Research, 41, 265-271.

Kulendran, N., \& Wong, K. K. (2005). Modelling Seasonality in Tourism Forecasting. Journal of Travel Research, 44, 163-70.

Law, R. (2000). Back-propagation learning in improving the accuracy of neural network-based tourism demand forecasting. Tourism Management, 21, 331-340.

Law, R., \& Au, N. (1999). A neural network model to forecast Japanese demand for travel to Hong Kong. Tourism Management, 89-97.

Lim, C., \& McAleer, M. (2001). Monthly Seasonal Variations: Asian Tourism to Australia. Annals of Tourism Research, 28, 68-82.

McElroy, T. (2008). Exact Formulas for the Hodrick-Prescott Filter. Econometrics Journal, 209217.

Mustafaraj, G., Lowry, G., \& Chen, J. (2011). Prediction of room temperature and relative humidity by autoregressive linear and nonlinear neural network models for an open office. Energy and Buildings, 1452-1460.

Muysal, M., \& Roubi, M. S. (1999). Artificial Neural Networks versus Multiple Regression in Tourism Demand Analysis. Journal of Travel Research, 38, 111-118.

Oh, C., \& Morzuch, B. J. (2005). Evaluating Time-Series Models to Forecast the Demand for Tourism in Singapore. Journal of Travel Research, 43, 404-13.

Osborn, D. R., Harevi, S., \& Birchenhall, C. R. (1999). Seasonal Unit Roots and Forecasts of TwoDigit European Industrial Production. International Journal of Forecasting, 15, 27-47. 
Pai, P.-F., Hung, K.-C., \& Lin, K.-P. (2014). Tourism demand forecasting using novel hybrid system. Expert Systems with Applications, 41, 3691-3702.

Patil, S., Tantau, H., \& Salokhe, V. (2008). Modelling of tropical greenhouse temperature by autoregressive and neural network models. Biosystems Engineering, 423-431.

Ravn, M. O., \& Uhlig, H. (2002). On adjusting the Hodrick-Prescott filter for the frequency of observations. Review of Economics and Statistics, 371-375.

Siegelmann, H., Horne, B., \& Giles, C. (1997 ). Computational capabilities of recurrent NARX neural networks. IEEE Transactions on Systems, Man, and Cybernetics, Part B (Cybernetics), $208-215$.

Song, H., Li, G., Witt, S. F., \& Athanasopoulos, G. (2011). Forecasting Tourist Arrivals Using TimeVarying Parameter Structural Time-Series Models. International Journal of Forecasting, 27, 855-69.

Song, H., Smeral, E., G. Li, \& Chen, J. L. (2013). Tourism Forecasting Using Econometric Models. In D. Buhalis, \& C. Costa, Trends in European Tourism Planning and Organisation (pp. 289-309). Bristol, UK: Channel View Publications.

Stock, J. H., \& Watson, M. W. (1999). Forecasting Inflation. Journal of Monetary Economics, 293335.

Stock, J., \& Watson, M. (2016). Dynamic Factor Models, Factor-Augmented Vector Autoregressions, and Structural Vector Autoregressions in Macroeconomics. In J. B. Taylor, \& H. Uhlig, Handbook of Macroeconomics (pp. Volume 2, 415-525). ELSEVIER.

Sun, E. W., \& Meinl, T. (2012). A new wavelet-based denoising algorithm for high-frequency financial data mining. European Journal of Operational Research, 589-599.

Yao, Y., Zhai, J., Cao, Y., Ding, X., Liu, J., \& Luo, Y. (2017). Data analytics enhanced component volatility model. Expert Systems with Applications, 84, 232-241.

Zhang, G., \& Qi, M. (2005). Neural network forecasting for seasonal and trend time series. European Journal of Operational Research, 501-514. 